\title{
International Medical Students' Congress of Bucharest, IMSCB 2017
}

Organizing Committee of IMSCB 2017.

IMSCB Meeting website http://ssmbucharest.ro/
Since 2004, the Medical Students' Society of Bucharest has been organising the National Congress for Medical Students and Young Doctors which has become an emblematic medical event, gathering high quality scientific works and the greatest names of the Romanian medical community.

This year, the students' society Figure 1. International Medical Students' Congress of Bucharest - Opening Ceremony took up the challenge of organising an international event in order to provide the medical students with the greatest opportunities that the medical world of the 21st century has to offer.

The trademark of this edition of the congress was multidisciplinarity.

Since we live in a time of tremendous progress in both technological and medical fields, we constantly need to adapt.

Furthermore, the medical fields need subspecialities and each medical case represents a more meticulous mission. Taking this

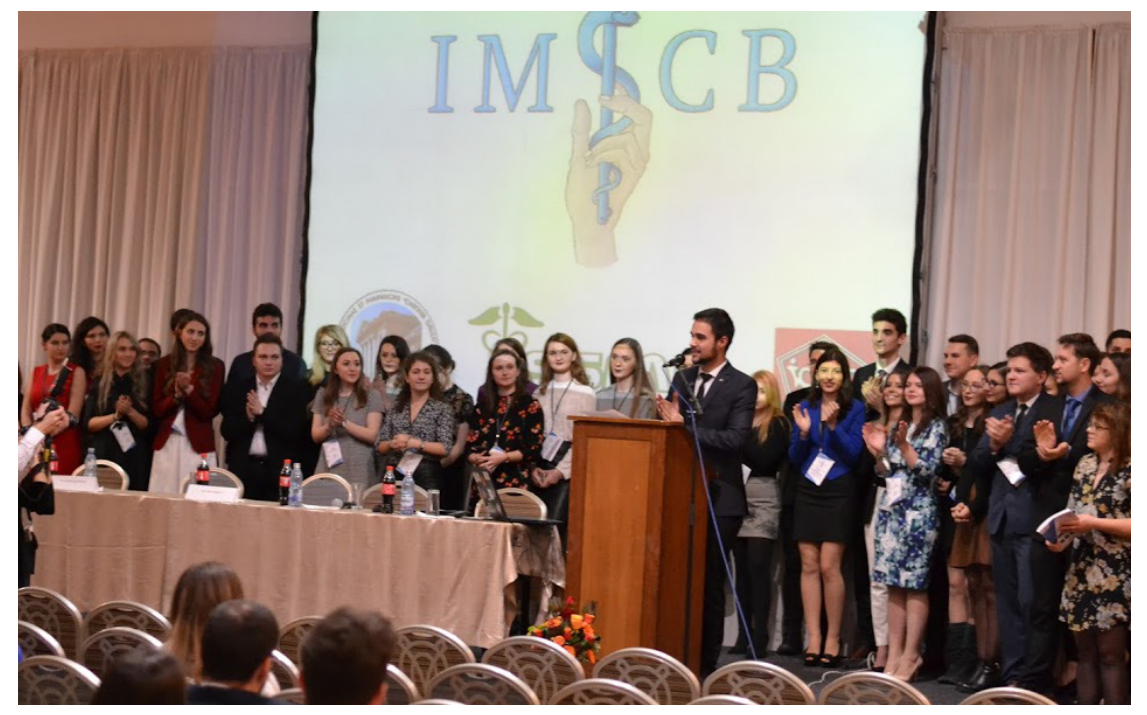

Figure 2. Workshop - Applications of $3 D$ Printing Technologies in the Field of Personalized Medical Implants.

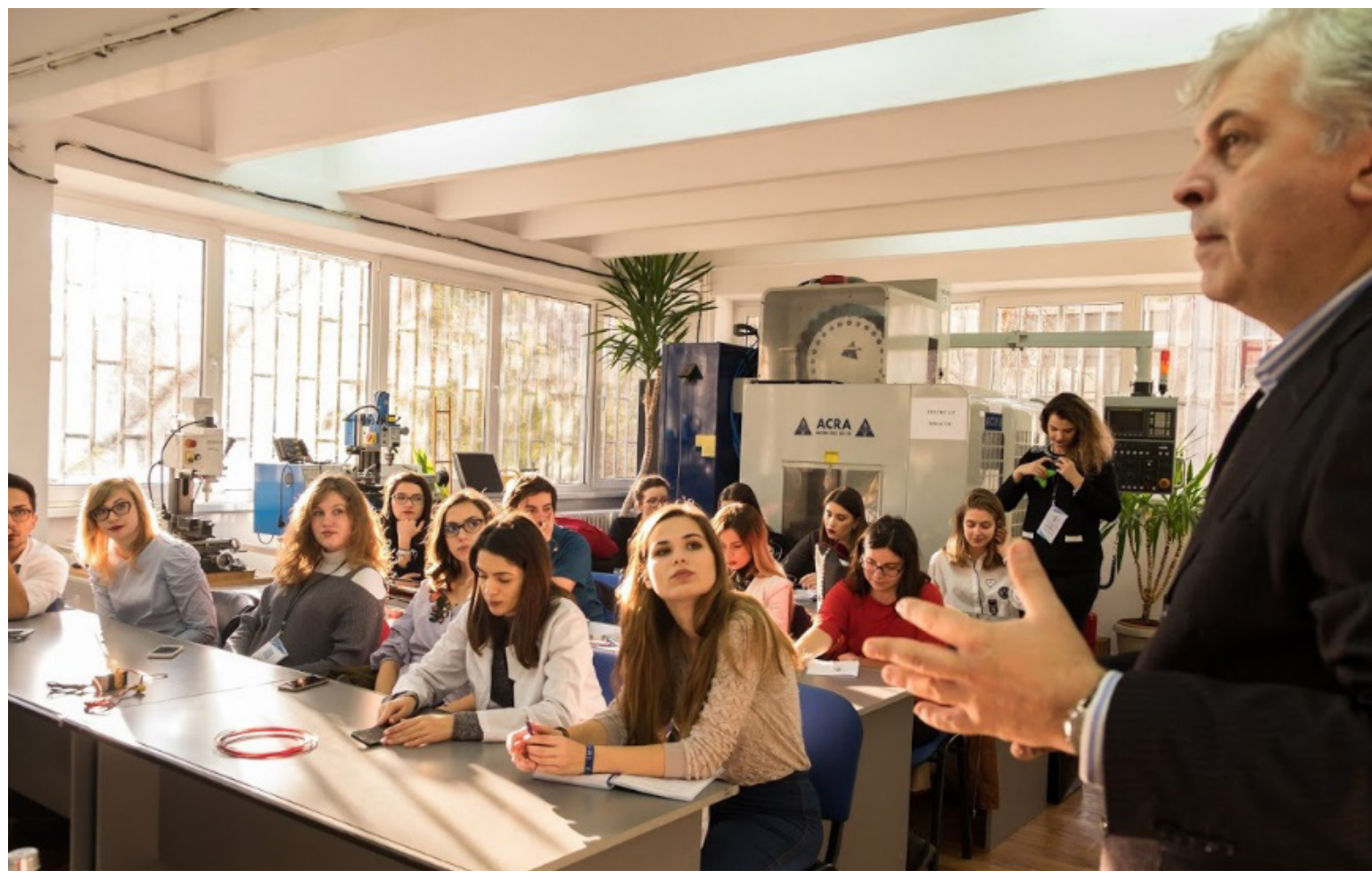

into consideration, the quest of achieving a comprehensive picture of the human organism can only be achieved in teams including multiple specialists. 


\section{Abstracts of the International Medical Students' Congress of Bucharest, IMSCB 2017}

01. Complement Mediated Regulation in Allergic Response Grigore M Rareș Gabriel ${ }^{1}$

Scientific Coordinators: Lecturer Sfrent Cornateanu Roxana Lucia $^{2}$

"Carol Davila" University of Medicine and Pharmacy, Bucharest, Romania; 2 Physiopathology and Immunology, Department I of Functional Sciences, "Carol Davila" University of Medicine and Pharmacy, Bucharest, Romania

Background: Firstly, we thought that the complement system's role in type I hypersensitivity reaction is just to amplify the classical IgE signaling. When mast cells are activated, they release a number of proteases that may cleave $C_{3}$ and $C_{5}$ to form $C_{3} a$ and $C_{5}$, also known as anaphylatoxins. Tryptase has been shown to do this in vitro. $\mathrm{C}_{3} \mathrm{a}$ and $\mathrm{C}_{5} \mathrm{a}$ then fix on their mast cell receptors and amplify the mast cell degranulation and cytokines formation. some recent studies suggest that allergens can activate the complement system mainly via classical pathway, but the alternative pathway may also occur. Methods: Serum samples from non-allergic with normal complement system patients, as well as from C2 deficient ones were incubated with ragweed and as control with veronal-buffered saline. It was measured the formation of two complement products ( $\mathrm{C} 3 \mathrm{bBbP}$ and $\mathrm{C}_{3} \mathrm{a}$ ). Results: In the normal complement serum group ragweed induced a strong C3bBbP formation and a significant C3a production as compared to the control group and to the $\mathrm{C}_{2}$ deficient serum. Conclusion: The mechanism of classical pathway activation by allergens has two explanations. According to Berrens et al. the activation of $\mathrm{C}_{1}$ is immunoglobulin-independent and is largely mediated by interactions between soluble degradation products in allergen extracts and unknown serum factors. The other explanation is based on studies performed with unrefined house dust extracts; it states that classical pathway activation by allergens is mediated by IgM antibodies directed to polysaccharide antigens in the extracts. A clinical importance of complement mediated regulation is the receptor desensitization. When a receptor is stimulated too often a signal is sent into the cell that makes it remove or internalize the receptor, so it can't be activated anymore. It is supposed that in mast cell activation syndrome (MCAS) the anaphylatoxins' receptors don't get desensitized. This would result in fast allergic reactions without IgE stimulation and chronic activation of the inflammatory response.

Keywords: type I hypersensitivity, C3a, C5a, C3bBbP, ragweed, complement system, allergic reactions, anaphylatoxins.

02. A Characteristic Case of Neurofibromatosis Type 1

Maher JM Sean', MD Eugenia Roza², Lecturer Raluca Teleanu ${ }^{2}$

"Carol Davila" University of Medicine and Pharmacy, Bucharest, Romania. 2. "Victor Comoiu" Paediatric Clinical Hospital, Bucharest, Romania

Background: Neurofibromatosis (NF) is a rare condition occurring in approximately 1 in 3000 individuals however it has a very distinctive clinical appearance. NF encompasses NF-1, NF-2, schwannomatosis and segmental neurofibromatosis, all of which are characterized by neuroectoderm tumors arising from a variety of organs and by autosomal-dominant inheritance. Case presentation: We present the case of a 7 year old girl with NF-1 who was referred to our paediatric neurology clinic by her G.P for the presence of Café Au Lait spots on her chest and back. Initial physical examination revealed the presence of $>10$ Café Au Lait spots with the largest measuring $13 \mathrm{~cm}$ in diameter and multiple freckles on her anterior thorax. Ophthalmological examination revealed 4 Lisch's nodules ( 2 on the right eye and 2 on the left eye). Soft tissue ultrasound of the precordium and left subscapular region showed nodules compatible with the diagnosis of neurofibromatosis which was further supported by lesions evident on head and spine MRI. Conclusion: This case highlights the characteristic appearance of NF which despite being among the most common inherited nervous system disorders worldwide, has no known cure or prevention. As NF represents a major risk factor for the development of malignancy early detection and constant monitoring are required with regular 6 month check-ups.

Keywords: Neurofibromatosis (NF), Neuroectoderm tumors, cafe au lait, lisch nodules.

\section{Acne Treatment with Isotretinoin - Correlations and Consequences \\ Mihailescu F Tessa1, 2, Bancescu C Bianca1, MD, PhD Cristina Nisipasu \\ "Carol Davila" University of Medicine and Pharmacy, Bucharest, Romania, ${ }^{2 S p a c e}$ and Aeronautical Medicine Institute, Bucharest, Romania}

Background: Acne represents a chronic skin disease which affects pilosebaceous units by blockage and/ or inflammation. Acne may appear as noninflammatory lesions, inflammatory lesions or a combination between the two of them. Isotretinoin is a medication, part of retinoids class, used to treat and prevent acne conglobata or acne which has not responded to other treatments. Materials and methods: In order to determine the side effects of the treatment with isotretinoin, 21 pacients (4 men and 17 women) were included into a retrospective study from May 2014 to September 2017, with ages between 14 and 31. Therefore, 0,5 $0.6 \mathrm{mg} / \mathrm{kg} /$ day PO divided BID were administered for approximately 8 months. At the first medical visit, before the beginning of the treatment, a clinical evaluation was made based on the appearance of acne conglobata, baseline monitoring was requested and the next medical visits were scheduled after one month and, then, three months. Results: We had two cases of psychiatric adverse effects such as anxiety and depressive symptoms and we ceased the treatment immediately. Another case presented hypertriglyceridemia and we reduced the isotretinoin dose. In an additional case, we noticed transaminase elevations and we had a similar approach by reducing the dose. The other patients' had a proper response to the treatment, without any relapse in acne after they finished this regimen. Conclusion: Isotretinoin is a safe and efficient drug for severe or moderate acne, as we get better results in treating this skin disease, if it is accurately and carefully monitored.

Keywords: acne, isotretinoin, adverse effects, skin disease.

04. An Anxious Physician at Risk of Sudden Death

David A Razvan', Constantinescu I. Gabriel-Bogdan', MD Serghei Cecoltan², Lecturer Silviu I. Dumitrescu³ "Carol Davila" University of Medicine and Pharmacy, Bucharest, Romania, $2 "$ Dr. Carol Davila" Central Military Emergency University Hospital, Bucharest, Romania, 3"Titu Maiorescu"University - Faculty of Medicne, Bucharest, Romania

Background: Sudden death is a non-traumatic, unexpected fatal event occurring within one hour of the onset of symptoms with a 
seemingly healthy subject. It is a major public heath issue, representing $25 \%$ of all cardiovascular deaths. Among the young people it had already taken a toll of 0.46-3.7 cases / 100000 persons / year, proving to be a dramatic psychological and social burden. Sudden death encompasses several etiology factors such as cardiomyopathy. The hypertrophic form of cardiomyopathy ( $\mathrm{HCM}$ ) is a disease characterized as an autosomal dominant trait, caused by mutations in cardiac sarcomere protein genes, that generates structural and functional abnormalities of the ventricular myocardium. Case presentation: We present the case of a 43-yearold physician, without noticeable cardiovascular risk factors, without a family or a personal pathological cardiac history, but extremely anxious. He presented himself at the hospital accusing fatigability generated by efforts for approximately the last six months, dyspnea and an episode of fast-paced palpitations. objective exam, laboratory tests, echocardiography - all within normal limits and a free pericardium. However, a 24-hour ECG Holter monitoring revealed a Non-Sustained Ventricular Tachycardia (NSVT) episode with 5 continuously ventricular beats and the results of the Bruce protocol were negative for ischemia. The surprise came during the second comprehensive echocardiography exam, made during the hospitalization, when we detected normal myocardial velocities, diastolic dysfunction grade II, pulmonary regurgitation gradient I, a posterior wall hypertrophy with the maximum thickness at the level of the papillary muscles of approximately $26 \mathrm{~mm}$, without intraventricular gradient at rest. However, the Valsalva maneuver showed a gradient of $64 \mathrm{mmHg}$ and on the stress test $>92 \mathrm{mmHg}$. Magnetic resonance imaging (MRI) exam confirmed the diagnosis of HCM with hypertrophied papillary muscles. Conclusion: The peculiarities of the case are the lack of ECG changes described in this very rare form of HCM located in the papillary muscles, the difficulty in identifying the hypertrophy of these structures and the importance of Valsalva maneuver in detecting an intraventricular gradient. Sometimes framing the patient in the anxiety pattern can superficially alter medical examination, but often small and usual maneuvers can help us make difficult diagnosis.

Keywords: hypertrophic cardiomyopathy, ECG Holter, pulmonary regurgitation, hypertrophied papillary muscles.

Aortic Valve Pneumococcal Endocarditis with Severe Periannular Complications: A Challenging Case

Găitan V Andreea $^{1,2}$, Lie V Delia',3, Associate Professor Adrian Molnar',2

"Iuliu Hatieganu" University of Medicine and Pharmacy ClujNapoca, Romania, " "Niculae Stăncioiu" Heart Institute of ClujNapoca, Romania, "'Children's Emergency Hospital Cluj-Napoca, Romania

Background: Pneumococcal endocarditis (PE) is a rare entity nowadays, responsible of 1 to $3 \%$ of native valve endocarditis cases. Due to a wide spectrum of clinical presentations, PE remains a diagnostic challenge and it is still associated with high mortality and severe complications. One of the most feared complications is uncontrolled infection, which spreads to the surrounding tissue resulting in periannular complications. This is mainly encountered in aortic valve PE where abscesses formed there may rupture internally with the subsequent development of aorto-cavitary fistulae (ACF). These abnormal communications create intracardiac shunts, which may cause clinical deterioration and hemodynamic instability of the patient. We present a rare and challenging case of aortic valve PE complicated with periannular abscess leading to extensive aortic root destruction and ACF. Case presentation: A 42-year-old male patient was admitted in our department after being transferred from The Infectious Disease Hospital due to worsening condition. The patient presented in our service with severe dyspnea at rest and orthopnea, fatigue and fluid retention. Physical examination revealed fever $\left(42^{\circ} \mathrm{C}\right)$, which began 4 weeks before, hypotension, tachycardia and a diastolic murmur in the aortic area. Paraclinical studies showed an inflammatory syndrome and elevated white blood count.
Hemocultures were positive for penicillin-susceptible Streptococcus pneumoniae. Transoesophageal echocardiography revealed a tricuspid aortic valve with severe endocardial lesions: right coronary cusp vegetation, ruptured aortic cusps, aortic root abscess and aorto-right ventricular fistula extended into the interventricular septum. Considering the patient's clinical status and the paraclinical findings, the diagnosis was established and urgent surgery was indicated. The patient underwent aortic valve and root replacement. Because of severe ostial lesions of the coronary arteries, their reimplantation was impossible, therefore triple coronary artery bypass grafting was done using the saphenous vein. Postoperative evolution was good, without remarkable events. The follow-up of the patient consisted in another 6 weeks of antibiotic treatment. Conclusion: This case reports the successful treatment of a rare, challenging case of $P E$ and highlights the importance of rapid, accurate diagnosis and early, appropriate treatment of the disease to prevent serious complications.

Keywords: pneumococcal endocarditis, aortic root abscess, aortocavitary fistulae.

\section{Carbapenemase Production in Clinically Isolated Enterobacteriaceae}

Matei A Cristian-Alexandru', Assistant Lecturer Tălăpan Daniela'

" "Carol Davila" University of Medicine and Pharmacy, Bucharest, Romania

Background: The number of nosocomial infections produced by carbapenem-resistant enterobacteriaceae is rapidly increasing, whereas the treatement options are severly narrowed. The purpose of this study is to establish the ratio of carbapenemaseproducing strains, as well as their type, among the carbapenemresistant strains. Materials and methods: The study was conducted among 61 carbapenem-resistant strains of Klebsiella Pneumoniae and Escherichia Coli isolated during the year 2016 from patients admitted to the National Institute of Infectious Diseases "Prof. Dr. Matei Balș", Bucharest. These strains were collected and stored on soft-agar tubes as their carbapenem-resistance was discovered in patient's antibiograms. In order to activate the dormant bacteria the strains were grown on lactose agar. To determine the type of carbapenemase produced, we ran the combination disk test, using a carbapenemase confirmation kit, according to Eucast Guidelines. The carbapenemases revealed by the test are Klebsiella Pneumoniae carbapenemase (KPC), Metallo-beta-lactamase (MBL) and oxacillinase- 48 (OXA-48). Results: A total of 38 strains were identified as carbapenemase producers, among the 61 carbapenem-resistant strains (62.3\%): 33 producing 0XA-48 $(49.25 \%), 4$ producing $\mathrm{MBL}(6.55 \%)$ and 1 producing $\mathrm{KPC}(1.64 \%)$. The carbapenem-resistance of the 23 remaining strains (37.7\%) could not be linked to the production of carbapenemases. Conclusion: During the year 2016, the most frequent cause of carbapenem-resistance among enterobacteriaceae was the production of carbapenemases, with $0 X A-48$ being the most recurrent. This finding is optimistic as OXA-48 has a weaker hydrolytic action upon carbapenems than KPC and MBL, which suggests a better prognosis.

Keywords: Enterobacteriaceae, carbapenemase, carbapenem, nosocomial, resistance.

\section{Circumcaval Ureter Ablation through a Laparoscopic Procedure}

Duca F.N. Stefania-Teodora', Costache R. C. AlexandruDan', Minea T Horia-Octav', Dumitrescu Nicoleta', Assistant Lecturer Bogdan Novac ${ }^{1,2}$

1"Grigore T. Popa" University of Medicine and Pharmacy, Iași, Romania, 2"Dr. C. I. Parhon" Clinical Hospital, Iași, Romania

Background: Although it is often considered as being caused by an improper ureteral development, a circumcaval ureter, more 
commonly described as a retrocaval ureter, is a congenital abnormality of the inferior vena cava. There are two types described, with a high loop or a low loop, both being mainly asymptomatic, with patients developing, depending on the degree of compression, either a partial right ureteral obstruction or recurrent urinary tract infections due to urinary stasis. Case presentation: We are presenting the case of a 57-year-old patient, admitted in the Urology Department of the Parhon Teaching Hospital, complaining for three months of right lumbar pain with antero-inferior radiation. A right retrocaval ureter was revealed using imaging investigations, which caused an obstruction, explaining the lumbar pain and the third degree right hydro nephrosis and hydro ureter. A laparoscopic uncrossing of the congenital malformation was performed, which consisted of the relocation of the ureter anteriorly to the inferior vena cava, accompanied by pyeloplasty. After the procedure, the preoperative symptoms have subsided and the postoperative period was uneventful. The patient was discharged with a right self-retaining ureteric catheter in situ for the next three months. Conclusion: Despite the small number of cases recorded in medical literature, this case is a strong argument for the importance of performing minimally invasive surgery and underlines the excellent outcome of these procedures.

Keywords: circumcaval ureter, congenital malformation, laparoscopic surgery.

08. Craniectomy - a Good Choice for Patients with Intracerebral Haemorrhage.

Bratu I Flavius, ${ }^{1,2}$, MD PhD Athena Cristina Ribigan³, Professor Ovidiu Alexandru Băjenaru',4, MD PhD Florina Anca Antochis "Carol Davila" University of Medicine and Pharmacy, Bucharest, Romania, 'University Emergency Hospital, Bucharest, Romania, 3Emergency Teaching Hospital, Bucharest, Romania, 4Fundeni Clinical Institute, Bucharest, Romania

Background: Haemorrhagic stroke is the result of blood vessel rupturing and bleeding into the surrounding brain. Intracerebral haemorrhage $(\mathrm{ICH})$ is one of the most devastating types of stroke, leading to severe disability ( $12 \%$ to $39 \%$ of the survivors achieving long-term functional independence) or mortality (40\% at 1 month and $54 \%$ at 1 year). As the blood accumulates, it compresses the surrounding cerebral tissue, leading to an intractable rise in intracranial pressure. Decompressive craniectomy (neurosurgical procedure in which part of the skull is removed) has proved to be a good choice in improving the outcome of ICH. Case presentation: A 38-year old male patient with medical history of severe arterial hypertension and left lenticular ICH (7 months prior to admission) was brought to the Emergency Room with symptoms and signs suggestive for an acute stroke: left central facial palsy (CFP), left side hemiparesis (Medical Research Council - MRC - grade 1/5), hyperreflexia and Babinski sign on the left side and severe dysarthria. The cerebral computed tomography (CT) scan showed a massive haemorrhage located in the right lenticulo-capsular region. There was no trombocytopenia or other coagulopathies and the neurosurgical exam established that the patient is a candidate for fronto-temporo-parietal decompressive craniectomy. After surgery, the patient was admitted to the Intesive Care Unit for 4 weeks (tracheostomy was performed and a nasogastric tube was used) and then transferred to the Neurology and Rehabilitation departments with neurological improvement. The subsequent CTs taken after surgery have shown progressive reduction in the herniation of the brain parenchyma, cerebral oedema and of the $\mathrm{ICH}$; the last $\mathrm{CT}$ scan revealed the absence of $\mathrm{ICH}$. The patient was discharged with left sided hemiparesis (1/5 MRC brachial and 2/5 crural), mild dysarthria, but conscious, complying and normal oriented in time and space. Conclusion: This case was chosen to be presented in order to highlight that decompressive craniectomy is a safe and effective procedure in selected cases of ICH and that appropriate management sustained by a multidisciplinary team may reduce the associated risks of morbidity and death.

Keywords: intracerebral haemorrhage, decompressive craniectomy

\section{Hypertension in Pheochromocytoma}

Lazăr G Diana-Georgiana', MD, PhD Oros Sabina Elena ${ }^{2}$

"Carol Davila" University of Medicine and Pharmacy, Bucharest, Romania, "2C.I.Parhon" National Institute of Endocrinology, Bucharest, Romania

Background: Pheochromocytoma is a catecholamine-secreting tumor that derives from chromaffin cells of adrenal medulla and the sympathetic ganglia. The lack of its detection can lead to morbidity and high level of mortality, as a result of cardiovascular compound and uncured malignity. Materials and methods: I carried out an observational, retrospective and descriptive study, based on a sample of 59 patients suffering from secondary endocrine hypertension, hospitalized in "C.I. Parhon" National Institute of Endocrinology, between 2014-2016. The data have been obtained from its database and from pathologic reports.

Results: 39 patients were women and 20 were men, aged between 50-70 years old. 54 patients have been diagnosed with pheochromocytoma and 5 patients with paraganglioma, 52 with extra-adrenal localization, 44 being unilateral. A number of approximately 38 patients had a previous pathologic cardiovascular condition. Seventeen patients have been suffering from hypertensive crisis. Out of the remaining patients without known previous cardiovascular pathology, 8 have been witnessing incipient sympthoms of hypertensive crisis, these being correlated with the diagnosis. 38 people have been previously diagnosed with hypertension, most of them Grade 2 or 3. Diagnosis has been suggesting by imaging for $37 \%$ of cases, $32 \%$ have been presented a hypertensive crisis and the rest of $31 \%$ have been suffering from abdominal pain, thrills, headache and sweating. Around $10 \%$ of patients have been identified with three genetic syndromes, 8 having MEN 2A, 2 patients with von Hippel-Lindau disease and 1 patient with neurofibromatosis. Cardiovascular complications occurred in 20 subjects. Eight patients presented with chronic heart failure and 5 patients presented left ventricular hypertrophy. Arrhythmia occurred in 6 patients. A small number of subjects suffered from hypertrophic cardiomyopathy (3 patients) and permanent atrial fibrillation ( 3 patients). Conclusion: Based on the obtained data, approximately $38 \%$ of patients without pathologic cardiovascular background have been diagnosed with hypertension and the hypertensive crisis was the way of pheochromocytoma presentation.

Keywords: pheochromocytoma, paraganglioma, cardiovascular complications, hypertensive crisis

\section{Matrix Metalloproteinases in Differential Diagnosis of Inflammatory Bowel Disease \\ Ungureanu G Razvan - George ${ }^{1}$, Professor Isabela Silosi \\ ' University of Medicine and Pharmacy, Craiova, Romania}

Background: The matrix metalloproteinases (MMPS) expression is altered in inflammatory bowel disease (IBD) but while their involvement is known, circulating concentrations of MMPs, a potential tool for diagnostic tests, have not been established.This paper aims serum quantitative investigation of MMP-3 and MMP-9 in patients with IBD compared with control group, for establishing their roles in the disease pathophysiology and as potential biomarkers in differential diagnosis. Materials and methods: The present study investigated levels of MMP-3, MMP-9 and CRP in serum samples of 67 patients, of which 46 with ulcerative colitis (UC) and 21 affected by Crohn's disease(CD). Parallel, we used a control group of 30 persons unaffected by $C D$ or UC. For dosage of MMP-3, MMP-9 and CRP, were used Invitrogen Corporation and INOVA-ELISA kits. Clinical disease activity was evaluated using a Truelove-Witts severity index TWSI for UC and the Harvey-Bradshaw severity index ( $\mathrm{HBI}$ ) for CD. Results: Serum levels of MMP-3, MMP-9 and CRP were significantly higher in IBD patients than in controls $(p<0.0001)$. In UC serum levels of MMP-9 showed significant correlation with TWI score $(r=0.308, p=0.037)$ but MMP-3 levels were statistically correlated only with the number of points 
obtained in the evaluation of disease activity by TWI score $(r=0.344, \quad p=0.029)$. In CD patients, MMP-9 concentrations correlated positively with $\mathrm{HBI}(r=0.608, p=0.039)$; no significant correlations were between concentrations MMP-9 and CRP $(r=0.246, p>0.05)$. Serum levels of $M M P-3$ in $C D$ patients were correlated better with indices of disease evaluated for this entity. Conclusion: The data of our study indicate the presence of correlations between the activity of inflammatory bowel disease and serum levels of matrix metalloproteinases. In CD patients serum levels of MMP-3 were correlated better with indices of disease (HBI and CRP) evaluated, while in patients with UC, was achieved better correlation with MMP-9. We can notice that, MMP3 and MMP9 concentrations are important indicators of inflammatory disease, which can be used in differential diagnosis of IBD.

Keywords: matrix metalloproteinases, inflammatory bowel disease, Crohn's disease. ulcerative colitis

11. Minimal Residual Disease Evaluation in Acute Promyelocytic Leukemia

Onofrei L Andreea', Miron N Victor Daniel', Florea N Roxana',2, Marin M Dragos-Andrei', Professor Daniel Coriu $^{1,3}$, Assistant Lecturer Mihaela Cîrstea ${ }^{1,3}$

"Carol Davila" University of Medicine and Pharmacy, Bucharest, Romania, ${ }^{2 " D r}$. Carol Davila" Central Military Emergency University Hospital, Bucharest, Romania, 3Fundeni Clinical Institute, Bucharest, Romania

Background: At one time a highly lethal malignancy, significant progress has transformed acute promyelocytic leukemia (APL) into the most curable form of acute myeloid leukemia. The use of alltrans-retinoic acid (ATRA) and chemotherapy as front-line therapy resulted in a cure rate above $80 \%$. Molecular monitoring of the minimal residual disease (MRD) by means of polymerase chain reaction $(P C R)$ is essential for detecting submicroscopic disease after therapy and for identifying patients with an imminent hematological relapse. Case presentation: A 33-year-old woman was admitted in January 2016 to Fundeni Clinical Institute-Center of Hematology and Bone Marrow Transplant. The complete blood count showed moderate anemia $(\mathrm{Hb}=8.1 \mathrm{~g} / \mathrm{dL})$, leukocytosis $(\mathrm{WBC}=103000 / \mathrm{mmc})$ with myeloblasts (7\%), atypical promyelocytes $(60 \%)$ on differential count and thrombocytopenia $(\mathrm{PLT}=37000 / \mathrm{mmc})$. Coagulation tests revealed disseminated intravascular coagulation. A diagnosis of APL was set and emergency treatment with ATRA was initiated. Bone marrow aspirate was markedly hypercelular $(90 \%)$ with diffuse infiltration by $>80 \%$ leukemic blasts that on immunophenotyping expressed CD33, CD13, CD38, CD45 and were negative for HLA-DR and CD34. Induction was started based on the AIDA protocol: oral ATRA 45 $\mathrm{mg} / \mathrm{m} 2 /$ day and intravenous bolus idarubicin $12 \mathrm{mg} / \mathrm{m} 2$ on days 2 , 4, 6 and 8. Two days later the patient developed the differentiation syndrome, a life-threatening complication of ATRA therapy. ATRA was discontinued and the symptoms improved with dexamethasone 1omg iv once every 12 hours for 5 days.Three courses of consolidation therapy according to AIDA protocol and maintenance therapy with 6-mercaptopurine and methotrexate were given. Molecular biology testing for monitoring MRD was performed every 3 months and revealed positive PML-RARA transcript at the follow-up in November 2016, molecular remission being diagnosed. Induction therapy using arsenic trioxide (ATO) was started. Currently, the patient achieved a new molecular complete remission and undergoes consolidation therapy with six arsenic trioxide courses supplemented by ATRA. Conclusion: The patient had a high risk of relapse taking into account the leukocyte count at the time of diagnosis. This case demonstrates that MRD evaluation is of utmost importance for patient prognosis and management. Early institution of salvage therapy in patients with APL presenting molecular relapse guarantees a better outcome compared to treatment at the time of hematological relapse.

Keywords: minimal residual disease, acute promyelocytic leukemia, salvage therapy, ATRA

\section{Multiple Sclerosis. Clinical Isolated Syndrome}

Ionescu C Ruxandra Florentina', Robert-Mihai I.L Enache ${ }^{1}$, Scientific Coordinators: MD PhD Maliia Mihai Dragos², MD Radu Lidia ${ }^{3}$

"Carol Davila" University of Medicine and Pharmacy, Bucharest, Romania, 2Emergency Teaching Hospital, Bucharest, Romania, 3Private Medical Practice of Internal Medicine, Bucharest, Romania

Background: Multiple sclerosis is a chronic disorder of the central nervous system characterized by recurrent episodes of inflammation and focal demyelination in multiple locations. The disease has genetic susceptibility and it consists in progressive axonal degeneration. In Romania multiple sclerosis has a prevalence of $35-40$ per 100,000 inhabitants and it is more frequent in women. Case presentation: A 15-year-old female, with no medical history (heredocolateral or personal) accused sensitivity disturbances of the left hemibody. She had suffered a minor injury to her left upper limb a week before coming to the appointment, but the performed $\mathrm{X}$-ray infirms any fracture. Afterwards, she complained about paresthesias in the distal portion of the left upper limb (glove). Two days after the injury, paraesthesias appeared in the left lower limb and in the left hemithorax. The clinical examination could not confirm noticeable neurological disturbances. After the first examinations, the diagnosis was demyelinating disease. As further results, hyperintensity lesions on T2/ FLAIR brain MRI were revealed, along with supratentorial demyelinating areas, as well as non gadolinofile areas. The MRI indicated intracordonal spinal lesions of the cervical spine, $C_{4}-C_{5}$, eccentric, with contrast media uptake and with active demyelinating lesion. After cerebrospinal fluid examination, the McDonald conditions were met (1.10 IgG index and oligoclonal bands absent) and the diagnosis of multiple sclerosis was sustained. Corticosteroid therapy (pulse therapy) was initiated, according to current clinical guidelines, with remission of sensitivity disorders. Subsequently, immunomodulatory therapy with $\beta-1$ a interferon was initiated, with favorable, persistent outcome after 6 years of therapy. The patient is still in remission (without relapses). Conclusion: This clinical case stands out due to its high lesional areas, associated with a non-specific symptomatology. Positive diagnosis was based on clinical and MRI appearance according to McDonald criteria. The acute treatment accelerated the disappearance of neurological conditions while the chronic immunomodulatory treatment stabilised the pathogenesis and prevented natural evolution (relapses and permanent neurological deficit).

Keywords: multiple sclerosis, MRI, $\beta-1 \mathrm{a}$ Interferon

13. New Treatment Strategies in Familial Hypercholesterolemia Akad H Nada', Donos I Madalina-Andreea', MD Adelina Tonita ${ }^{2}$, Associate Professor Florin Mitu ${ }^{1,2}$, MD Paul Alexandru Simion ${ }^{2}$

"Grigore T. Popa" University of Medicine and Pharmacy, lași, Romania, ${ }^{2 M e d i c a l ~ R e c o v e r y ~ H o s p i t a l, ~ l a s s i, ~ R o m a n i a ~}$

Background: Familial hypercholesterolemia (FH) is a common monogenic dyslipidaemia causing premature cardiovascular disease due to lifelong elevation of plasma levels of low density lipoprotein-cholesterol (LDL-C). If left untreated, patients with hererozygous $\mathrm{FH}$ ( $\mathrm{HeFH}$ ) typically develop coronary artery disease before the ages of 55 years. Case presentation: We present the case of a 51 years old woman with an important family history of dyslipidemia (father, sister and her only son are diagnosed with HeFH), hypertensive, dyslipidemic, with symptomatic plurivascular lesions (stable angina pectoris and transient ischemic attacks) treated with triple aortocoronary by-pass, and endarterectomy for left common and left internal carotid artery, and with percutaneous angioplasty with stent implantation on the right common carotid artery. Currently she is asymptomatic, and has come for her regular follow-up in the cardiology department. At admission the patient was hemodynamically stable $(\mathrm{BP}=130 / 80 \mathrm{mmHg})$, in sinus rhythm $64 \mathrm{bpm}$, and without any signs 
of ischemia on the EKG. Ecocardiography revealed a nondilated left ventricle, with normal segmentary and global contractions, with a moderate concentric hypertrophy, and with a type I diastolic dysfunction, having an ejection fraction of $52 \%$. The biochemistry showed a total cholesterol of $643 \mathrm{mg} / \mathrm{dl}$ and LDL-C $566 \mathrm{mg} / \mathrm{dl}$. We recommended a combination of "Mediteranean diet" and "Portfolio diet" so as the cholesterol intake does not exceed $250 \mathrm{mg} /$ day. The medication consisted in rosuvastatin $40 \mathrm{mg} /$ day, ezetimibe $10 \mathrm{mg} /$ day, aspirin $75 \mathrm{mg} /$ day, bisoprolol $10 \mathrm{mg} /$ day, perindopril $5 \mathrm{mg}$ /day and indapamide $1,5 \mathrm{mg}$ /day, the lipid profile decreasing slowly during the follow-up with a maximum of $70,84 \%$ after one year. Conclusion: $\mathrm{FH}$ needs to be diagnosed early and promptly treated in order to prevent major cardiovascular events that usually appear in the 4 th to 5 th decades of age. A cholesterol restrictive diet with the addition of aliments known to reduce cholesterol like almonds, soy proteins, sterols and phytosterols is recommended. Unfortunately aggressive treatment is also required and current guidelines suggest the addition of ezetimibe to a statin in order to reach LDL-col under $70 \mathrm{mg} / \mathrm{dL}$, but often times this target is not achieved. Novel therapy with PCSK-9 inhibitors have better reduction in lipid profiles, but they are expensive or are accessible only through clinical trials.

Keywords: familial hypercholesterolemia, PCSK-9 inhibitors, statin, ezetimibe

\section{Primary Spontaneous Pneumothorax}

Kerezsi C Alexandra Diana', Taran G Camelia-Anamaria', Vancea G Andreea-Paula', Lecturer Mekereș Florica'

'University of Oradea, Faculty of Medicine and Pharmacy, Oradea, Romania

Background: Pneumothorax is a complex syndrome that occurs secondary to the penetration of air into the pleural cavity. Among the forms of pneumothorax, the spontaneous one is more difficult to define, because the causes of it's occurrence are unpredictable. There are no clinical signs or symptoms in primary spontaneous pneumothorax until a bleb ruptures and causes pneumothorax; typically, the result is acute onset of chest pain and shortness of breath. Case presentation: We present the case of a 26-year-old male patient presenting on 02.01.2016, 01:26 in the Clinical County Emergency Hospital, Emergency Room Service. He accused shortness of breath, intense toracoalgies, irritable cough with sudden onset with progressive worsening. The man reports that he has woken up from sleep because of these symptoms. It should be noted that the patient is a smoker and has no significant pathological history, with no history of exposure to other noxes. After consultation and specialist treatment the patient is hospitalized. After a radiographic examination, we can see a massive air leakage collection in the right lung field. The CT scan confirms that it is a right massive pneumothorax. In the clinical and imaging context, a minimal right pleuroctomy is performed with a minimal incision in the $\mathrm{V}$ intercostal space. A large amount of air is evacuated. Post-operative, clinical and radiological evolution is partially favorable, with persistent aerial loss. On the fifth day, a second intervention is performed where an apical upper right lobe resection (bulectomy) is performed. As a result, the evolution of the patient is favorable, without complications. Conclusion: Spontaneous pneumothorax occurs mainly in young people without a history of lung disease, but smoking tobacco and/or marijuana induces a significantly higher risk and increases the likelihood of recurrence.

Keywords: primary spontaneous pneumothorax, intense thoracalgia, dyspnea

\section{Severe Congenital Scoliosis Surgery.}

Tarta N Amalia', Coauthors: Szatan G Dalma-Zsuzsa', Lavinia-Maria G Tusan', Lecturer Mekeres Florica'

IUniversity of Oradea, Faculty of Medicine and Pharmacy, Oradea,
Background: Scoliosis affects $2 \%$ of females and $0.5 \%$ of men in the general population and it is defined as an evolutionary deviation of the spine that can be genetically inherited, but often occurs without a specific cause of approximately $80 \%$ of the cases (idiopathic scoliosis).A relatively new surgical method has been discovered in the treatment of severe scoliosis, which consists in the implantation of a metal system of rods and hooks, which by the force of distraction and compression applied to the spine will correct its curves and stabilize it by skeletal fixation. This method of treatment is required in the most severe cases of scoliosis with a curvature over 40 Cobb degrees. Case presentation: In 2006, A.T, a Romanian 12 years old patient was evaluated by a doctor from Head Spine Surgery department in Zurich, Switzerland and after the MRI, CT investigations, diagnosed with: Congenital Thoracic Lordoscoliosis-convex right with scoliotic pelvis and decompensated posture. The patient was presenting severe thoracic curvature of 90 Cobb degrees between Th5 and Th12. Convex left with a high thoracic curvature of 34 Cobb degrees between Th1 and Th4.The preparation for surgery started by proceeding with the Halo Orthosis Immobilization on January 26th 2010. After the first surgery, one the post-operative complications appeared due to the compression of the spinal cord by a screw at the Th12 vertebrae: the patient presented paraparesis of the lower limbs, right more than left followed by sensory loss in the $L_{3}, \mathrm{~L} 4$ dermatomal area with neuropathic pain. Conclusion: For the seven years that passed since the surgery, the only post-surgery complications that remained are the disrupted tactile perception between the areas of L3-L4 left side and motor instability of the right ankle following the paraparesis.

Keywords: spine, congenital scoliosis, neurological disorder, scoliosis surgery, complications

\section{Signet Ring Cell Carcinoma of the Ampulla of Vater: A Very Rare Malignancy}

Constantinescu I Angela', Sandu E Gabriel', Voloagă V Ioana', Padure I.T. Rares Cristian', MD Musina Ana-Maria², MD Hutanu Ionut ${ }^{2}$

"Grigore T. Popa" University of Medicine and Pharmacy, lassi, Romania, 2Regional Oncology Institute, Iași, Romania

Background: Cancer of the ampulla of Vater is a rare entity accounting for only $0.2 \%$ of all gastrointestinal malignancies and $<6 \%$ of all periampullary cancers. Signet ring cell carcinoma("SRCC") of the Vater's ampulla is extremely rare, and only 15 cases had previously been described in the literature. The World Health Organization defined SRCC as a special type or a variant of gastrointestinal adenocarcinoma. SRCCs may exist alone or coexist with any other types of malignant gastrointestinal tumors. Case presentation: We present the case of a 67 year old male, who was admitted to the local hospital in Pascani, three weeks after showing signs of the following symptoms: jaundice, hyperchromatic urine, acholic stools, fatigabilty and astenia. The paraclinical investigation (an abdominal Magnetic Resonance Imaging ) raised the suspicion of a tumoral mass localized on Vater's Ampulla. The patient was then redirected to the Regional Oncology Institute in laşi. At admission the following were present: anemia, cholestatic and hepatocytolytic syndrome and tumoral markers within normal limits. The endoscopic examination detected a permeable piloric sphincter, a hyperemic duodenal bulb, a healed ulcer and in the second part of the duodenum, corresponding to the topography of the Ampulla of Vater, a protrusive submucous mass. During the surgery, a tumoral mass was found inside the Ampulla, primarily affecting the Wirsung's duct and even extending to the common bile duct as well as to the duodenal wall, which was why a whipple type cephalic pancreaticoduodenectomy and a Witzel jejunostomy were in order. The microscopic examination confirmed the diagnosis of a malignant neoplasia: signet ring cell adenocarcinoma of Vater's ampulla, associated with a minor component of poorly differentiated, intestinal type, ductal adenocarcinoma. Conclusion: Because of the unfavorable prognosis associated with an 
ampullary cancer, aggressive surgical treatment should be offered to the patient at a center that has performed a high volume of pancreatic surgery and the Whipple operation. Even though there have only been a few registered cases of adenocarcinoma of the ampulla, the results after radical surgical intervention have been improving over time. During recent decades, 5-year survival rates ranged from $20-61 \%$, averaging higher than $35 \%$.

Keywords: ampulla of Vater, signet ring cell carcinoma, pancreatoduodenectomy

17. The Challenges of Diagnosing and Treating ANCA Systemic Vasculitis

Manaila I Maria', MD Valentina Esanu²

"Grigore T. Popa" University of Medicine and Pharmacy, lași, Romania, 2Pneumology Clinical Hospital, Iași, Romania

Background: The primary systemic vasculitides are heterogeneous, multi-system disorders characterized by inflammation and necrosis of small and medium blood vessels. Their aetiology is unknown and they are often associated with anti-neutrophil cytoplasmic antibodies (ANCA). Studies from various countries show that the overall annual incidence of primary systemic vasculitis is approximately 20 cases per million. Case presentation: A 48-year-old woman reported to the Pneumology Hospital with dyspnea, asthenia, fatigue, joint aches, dry cough. Chest examination revealed basal crackles in the right lung and the chest radiography revealed nodular opacities, varying in size, with a confluence tendency, some excavated. Blood tests showed an important inflammatory syndrome and anemia. The patient was diagnosed with right pneumonia for which she received treatment Dyspnea and joint pain persisted. X-Ray performed after treatment showed a stationary radiological aspect. A chest CT highlighted bilateral multiple nodules, some ground glass opacities, others with peripheral contrast enhancement. Both bronchoscopic examination and bronchial aspirate revealed no abnormalities. Bronchial biopsy showed reduced chronic inflammatory infiltrate. Corroborating the results of laboratory investigations with the clinical symptoms, the probability of vasculitis was high and the patient was sent to Rheumatology for special investigations. In evolution, the patient presented external popliteal sciatic nerve paresis and subcutaneous nodules. Perinuclear ANCA (p-ANCA) were present, cytoplasmic ANCA ( $c$ ANCA) were absent. The patient started treatment with oral corticosteroid and cyclophosphamide.The treatment resulted in favorable development of the respiratory, neurological and articular symptoms, decreased levels of p-ANCA. The peculiarity of the case is represented by the complications of the corticosteroid therapy: the patient developed Cushing's syndrome and diabetes. Conclusion: Although it is a rare disease which is treated by rheumatologists, due to affecting multiple systems vasculitis should not be forgotten in the differential diagnosis by physicians from various specialties: pulmonology, dermatology, nephrology, neurology, otorhinolaryngology.

Keywords: systemic vasculitis, ANCA, anti-neutrophil cytoplasmic antibodies, Cushing's syndrome

18. The Employment of Molecular Biology in the Diagnosis of an Atypical Synovial Sarcoma

Rusu C Stefan 1,2,3, Cocuz G Iuliu Gabriel', Ovidiu S. Cotoi4, MD Cristi Marin ${ }^{2}$

"University of Medicine and Pharmacy, Târgu Mures, Romania, 2"Ambroise Pare" Teaching Hospital Boulogne-Billancourt, France, 3 Clinical County Hospital Tîrgu Mures, Romania, ${ }^{4}$ County Emergency Hospital, Tirgu Mures Romania

Background: Synovial Sarcoma (SS) is a rare malignant mesenchymal tumour, which may occur at any age (increased frequency in teenagers and young adults) with an equal distribution between the sexes, involving, most frequently, the deep soft tissues of the lower and upper extremities, often in a juxta-articular location. The microscopic aspect is dominated by a variable degree of epithelial differentiation, including gland formation and the molecular profile shows a specific chromosomal translocation $\mathrm{t}(\mathrm{x} ; 18)(\mathrm{p} 11 ; \mathrm{q} 11)$ which leads to formation of a $\mathrm{SS} 18$ SSX fusion gene. Clinically, it appears like a mass, often painful. Case presentation: We report a case of a 40-year-old man that presents a tumour on the right foot. After the surgical excision, the unfixed fragment was processed using standard histological techniques. Immunohistochemical staining was used for the positive and differential diagnosis. On cut-surface, the tumour, measuring $6.5 \times 4 \times 4.5 \mathrm{~cm}$, appears as a nodular, white bulging mass, largely ulcerated and focally hemorrhagic. It consists of a monomorphic cell proliferation, essentially spindle cells, with hyperchromatic nuclei and sparse cytoplasm arranged in short fascicles or spread in all directions, associated with an epithelial component, organized in tubulo- glandular structures. The tumour infiltrates as nodules or fascicules the dermis, hypodermis, the soft tissues and also the muscular layer, being extended beyond the deep surgical limit. The mitotic rate varies, showing focally over 20 mitosis/10 HPFs. Immunohistochemistry shows focal and low positivity for Antibodies antiEMA, $\mathrm{CK} \mathrm{AE}_{1} / \mathrm{AE}_{3}$ in the areas of spindle cells' components but highly expressed in the epithelial structures. Low positivity was shown for the antibody PS 100 and negativity for $\mathrm{CK}_{20}, \mathrm{CD}_{34}$, chromogranin and desmin. Considering the PS100 positivity, the probability of malignant peripheral nerve sheath tumour with glandular differentiation was evocated. The final diagnosis is SS biphasic grade 3, confirmed by the rearrangement of the gene $\mathrm{SS}_{18}$ identified here by RT-PCR technique. Conclusion: The particularity of the case consisted in the overlapped histological and immunohistochemical profile between SS and other tumours. The RT-PCR technique was essential for final diagnosis. Molecular biology shows its effectiveness in the management of the tumours. It's necessary having a reference center that can corroborate the diagnosis of ambiguous cases.

Keywords: Synovial Sarcoma biphasic, molecular biology, RT-PCR

\section{Two Faces of Preeclampsia}

Smilga V Santa', MD PhD Anna Miskova

'Stradinšs University, Riga, Latvia, Maternity Hospital, Riga, Latvia

Background: Preeclampsia complicates approximately $3 \%$ of pregnancies. It manifests after 20th week of gestation. Clinical signs include proteinuria and hypertension which could be accompanied with edema and other symptoms. Atypical preeclampsia with proteinuria or hypertension and cerebral symptoms, elevated liver enzymes, thrombocytopenia, hemolysis has been described, also. Case presentation: 25 year old patient, G1 in 37th week of gestation, was referred to emergency department (ED) due to elevated blood pressure for 5 days, pitting edema of feet and lower legs, weight gain, headache, proteinuria of $5 \mathrm{~g} / \mathrm{L}$. During last week the patient gained 10 kilograms. Patient was admitted with severe form of preeclampsia and induction of labour was initiated. Repeated urine analysis suggested proteinuria of $20,29 \mathrm{~g} / \mathrm{L}$. Urinary output for last 6 hours was 300 mililiters, suggesting oliguria. The patient complained of headache and facial edema. Seizure prophylaxis was initiated and decision to perform Cesarean section was made due to uneffective induction of labour to prevent progression and complications of preeclampsia. 29 year old patient, $\mathrm{G} 1$ in $41+1$ weeks of gestation, presented to ED with irregular pain in abdomen. She had no past medical history. During antenatal appointments, she was normotensive and did not have proteinuria. When regular pain ir lower abdomen started patient was transfered to Delivery ward. Amniotomy was performed and meconial amniotic fluid discharged. Afterwards cardiotocography with ST segment analysis was initiated. Due to slow progression labour augmentation with oxytocin was started. Patient complained about few seconds long eye blinking from light and difficulty turning head. Arterial blood pressure was $130 / 110 \mathrm{mmHg}$. Patient developed 30 seconds long 
seizure and was unconscious afterwards. Only then patient received magnesium sulphate and diazepam. Fetus developed acute dystress, labour could not be finished by operative vaginal delivery. The patient was transferred to surgical ward. Emergency Cesarean section was performed and central placental abruption diagnosed. The patient received seizure prophylaxis for 24 hours. Conclusion: It is easy to start appropriate treatment if there are classical signs of preeclampsia. In case of atypical preeclampsia it takes clinical knowledge and suspicion to start appropriate treatment. It is important because undiagnosed preeclampsia leads to complications for both mother and fetus.

Keywords: typical preeclampsia, atypical preeclampsia, hypertension, proteinuria

\section{Upfront Radiosurgery for Facial Nerve Schwannomas}

Jigoranu C.C Raul Alexandru', Scriminti I Diana Gabriela', Professor Marc Levivier ${ }^{2,3}$, MD Beatrice Concalves Matos03,4, MD Constantin Tuleasca ${ }^{2,3}$, MD Jean-Nicolas $\mathrm{Comps}^{2,3}$

"Grigore T. Popa" University of Medicine and Pharmacy, laşi, Romania, "2Neurosurgery Service and Gamma Knife Center, Lausanne University Hospital, Switzerland, 3University of Lausanne, Faculty of Biology and Medicine, Lausanne, Switzerland, 4Department of ENT surgery, Lausanne University Hospital, Switzerland

Background: Schwannomas represent $10 \%$ of all primary intracranial tumors. Ninety percent of them involve the VIII-th cranial nerve. For remaining $10 \%$, only less than $2 \%$ are localized on the VII-th, facial nerve. Furthermore, they represent most common primary tumor of this nerve. The gold standard treatment of facial nerve schwannomas (FNS) was considered surgery for decades. More recently, during the past two decades, new treatment modalities have emerged. As minimally invasive alternative, radiosurgery and particularly GK (Gamma Knife) has become increasingly used. In this context, we report our small case-series with GK in this pathology, over a period of 7 years. The patients have received a rigorous periodical follow-up, including clinical and radiological. Materials and methods: All patients were symptomatic pretherapeutically, with facial palsy or facial hemispasm. Three patients received a CK single fraction and 1 patient was treated with a "volume staged" radiosurgery, due to anatomical extension (geniculate ganglion, internal acoustic meatus and cisternal parts), dose overlap between the cochlea and the geniculate ganglion (if single fraction GK). Results: We treated 4 patients, mean age 44.25 years ( 3 female, 1 male). The mean follow-up was 31.8 months (median 36, range 3-60). Two cases presented with facial palsy and the other two with facial hemispasm. The mean gross tumor volume (GTV) was $406.6 \mathrm{~mm}^{3}$ (median $470 \mathrm{~mm}^{3}$, range $30-638 \mathrm{~mm} 3$ ). The prescribed dose was 12 Gy at the margin. Two patients improved and 2 were stable after GKS. One facial palsy, with a preoperative HB grade of $\mathrm{VI}$ dramatically alleviated and passed to a HB grade II. One case with a facial hemispasm had a HB grade going from III (preoperatively) to II (postoperatively). Auditory assessment was stable. Tumor volume was stable in one case and decreased in 3 patients. Conclusion: We present a small series of rare pathology of FNS, treated with upfront GK. In our experience, with small doses, by analogy with vestibular schwannomas, we obtain a high rate of tumor control (all patients). Clinical outcome was improved in two patients and stable in two. We conclude that GK can be a valuable alternative to open microsurgery in this rare pathology.

Keywords: Functional Recovery, Gamma Knife Radiosurgery, Facial Schwannoma

21. A Case of High Grade Stromal Endometrial Sarcoma Presented as a Giant Tumour

Pandele I Gabriel-Robert', Dugăeșescu D Monica', Lecturer Potecă Anca-Gabriela'
1"Carol Davila" University of Medicine and Pharmacy, Bucharest, Romania "Prof. Dr. Panait Sîrbu" Clinical obstetrics and Gynecology Hospital, Bucharest, Romania

Background: Endometrial stromal sarcoma is a rare malignancy of the uterus, comprising around $1 \%$ of all uterine malignant tumours. It usually extends into the myometrium, then into the serosa, and rarely can be seen as a nodule. The tumour invades blood and lymphatic vessels. Frequent symptoms are bleeding, pelvic pain, enlarged uterus. It is considered to have a favourable evolution, recurrence is encountered in one third of the cases. The treatment is hysterectomy and bilateral salpingo-oophorectomy. Case presentation: We present a case of a 54 year-old female patient who complained about metrorrhagia. Vaginal examination revealed a closed cervix, an enlarged firm uterus whose upper limit exceeds the umbilicus. Speculum examination showed a normal cervix. Colposcopy evidentiated hypertrophic cervix and atypical vascularisation. Pulmonary $\mathrm{X}$-ray indicated areas of opacity around the hilum, adjacent to the bronchi and blood vessels. Intraoperatory, a red tumour measuring $23 / 26 \mathrm{~cm}$ with areas of necrosis and hemorrhage was removed and sent for preliminary diagnosis. Microscopy revealed malignant cells resembling carcinomatous type. Total hysterectomy and bilateral salpingo-oophorectomy were performed. The body of the uterus measured $25 / 21 / 13 \mathrm{~cm}$ and had a cavity totally occupied by a white tumour showing haemorrhage and necrosis. The histopathological report pointed out a proliferation composed of round and spindle cells, atypical mitoses and cytonuclear atypia in the corpus of the uterus. The pattern was generally perivascular, showing areas of necrosis and haemorrhage. Microscopic, the cervix had a chronic inflammation, squamous epithelium with signs of malignancy in the inferior part. Immunohistochemistry confirmed the origin of the proliferation. The tumour was CD10, Ki67, Actin and Progesterone Receptors positive. The final diagnosis was high grade endometrial stromal sarcoma associated with high grade cervical intraepithelial neoplasia (CIN-2). The evolution of the patient was favourable after surgery. Conclusion: A giant uterine tumour is generally a fibroma. This case is special because although the clinical examination suggested a fibroma, more perfomant tests were necessary for a complete diagnosis. The association of endometrial stromal sarcoma with high grade cervical intraepithelial neoplasia is not common.

Keywords: giant tumour, endometrial stromal sarcoma, cervical neoplasm, hysterectomy

\section{A Mixed Glandular and Squamous Lesion of the Cervix} Dugăeșescu D Monica', Pandele I Gabriel-Robert', Lecturer Potecă Anca-Gabriela'

"Carol Davila" University of Medicine and Pharmacy, Bucharest, Romania, "Prof. Dr. Panait Sîrbu" Clinical obstetrics and Gynecology Hospital, Bucharest, Romania

Background: The cervical cancer is the second most common malignancy for women and is generally associated with human papillomavirus infection. There are plenty of HPV genotypes, but only a part of them can cause malignant tumours. Tumorigenesis involves many steps and other risk factors such as DNA mutations are necessary. Cervical intraepithelial neoplasia is a premalignant phase and comprises three stages $\left(\mathrm{CIN}_{1}, 2,3\right)$. $85 \%$ of the cervical tumours are squamous cell carcinoma of the cervix, while adenocarcinoma is seen in $10-15 \%$ of all cases. Case presentation: 64 year-old female presented for postmenopausal bleeding. She was clinically diagnosed with cervical polyp. The BabeșPapanicolau test revealed atypical glandular cells that suggested a neoplasia. HPV genotyping was performed and type 18 was evidentiated. The polyp was surgically removed and sent to the pathology laboratory. Endometrial aspiration was also performed. The histopathological exam indicated endocervical adenocarcinoma in situ. The patient underwent a total hysterectomy and bilateral salpingo-oophorectomy . The evolution was favourable. Macroscopic, the cervix measured $4 / 3 / 3,5 \mathrm{~cm}$ and the body of the 
uterus 3,5/2/5. A subserosal nodule and a polypoid lesion inside the uterine cavity were evidentiated. The histopathological exam of the cervical lesion indicated a glandular proliferation that had a complex architecture and cytonuclear atypia. Goblet cells were frequently seen. This lesion was associated with high grade cervical intraepithelial neoplasia ( $\mathrm{CIN} 2$ and $\mathrm{CIN}_{3}$ ). The endometrium was atrophic and presented cystic glandular dilation. The intracavitary polypoidal lesion was also made of cystic glandular dilations and consistent stroma. The subserosal nodule consisted of highly intricated fibers and areas of hyalinization and calcification. Immunohistochemistry was performed and the cervical lesion was positive to CEA, p16 and Ki67 markers. The final diagnoses were endocervical microinvasive adenocarcinoma intestinal type, high grade cervical intraepithelial neoplasia and subserosal leiomioma. The patient also presented ovarian serous cysts and right inguinal hernia. Conclusion: The most frequent lesion associated with HPV infection is squamous cervical cancer. Our patient had a mixed lesion of the cervix: intestinal type endocervical microinvasive adenocarcinoma associated with high grade cervical intraepithelial neoplasia.

Keywords: cervical adenocarcinoma, Human Papilloma Virus, cervical intraepithelial neoplasia, squamos cell carcinoma

\section{A Rare Case of Ichthyosis Uteri}

Sandu E Gabriel', Florea C Cristina-Maria', Hulubencu S Andreea Luana', Teodorescu-Soare E. G. Eugen Mihail', MD PhD Hutanu Ionut ${ }^{1,2}$

"Grigore T. Popa" University of Medicine and Pharmacy, Iași, Romania, ${ }^{2}$ Regional oncology Institute, Iași, Romania

Background: Ichthyosis Uteri is an extremely rare disease in which the entire endometrial surface is replaced by stratified squamous epithelium. First described as an endometrial response to iatrogenically-introduced caustic substances, similar changes have since been described associated with some inflammatory conditions of the endometrium. Ichthyosis Uteri's potential genesis is still uncertain, as well as its neoplastic potential. Case presentation: The 67 year old patient, with known cardiovascular pathology (terminal aorta thrombosis, critical ischemia of the lower limbs with axillofemoral bypass surgery), is diagnosed with cervical neoplasm stage FIGO IIA on February 2016. The anatomopathological examination revealed the presence of a poorly differentiated squamous cell carcinoma, which invaded more than half of endocervix's wall and extended towards the isthmus, the tumoral process developing on extended exocervix high-grade squamous intraepithelial lesions (HCSIL) with the infiltration of some vasculo-lymphatic spaces. Type $\mathrm{C}_{1}$ hysterectomy with bilateral pelvic lymphadenectomy stage 1 and 2 was performed and the procedure combined with the anatomopathological examination allowed for the diagnosis of Ichthyosis Uteri to be set. In October 2016, the patient was admitted at Regional Oncology Institute Iaşi for having the adjuvant treatment administered, as loss of appetite was present( $-3 \mathrm{~kg} / 10$ months). During vaginal valve examination no signs of tumoral growth were recorded. The microscopic examination established that no pathological variations were present and the Computed tomography detected no metastatic lesions. It is decided for the patient to undergo a treatment consisting of radiochemotherapy, concomitant with high dose rate brachytherapy and chemotherapy. Conclusion: Ichthyosis Uteri can be diagnosed only after hysterectomy, as patients don't accuse any specific symptoms. Due to the rarity of the condition, its clinical significance is not completely elucidated so far and there is insufficient evidence to suggest the intrinsic neoplastic potential of Ichthyosis Uteri.

Keywords: ichthyosis uteri, HCSIL, hysterectomy
Guțuleac Nicolae Valeria', Professor Maria Sajin², MD Adrian Dumitru², MD Diana Secara²

" "Carol Davila" University of Medicine and Pharmacy, Bucharest, Romania, 2Department of Pathology, Emergency Teaching Hospital, Bucharest, Romania

Background: Acephalus Acardia is extremely rare complication of multiple pregnancies and very uncommon congenital malformation disclosed in fetuses and newborns. It is among the most severe malformations described in fetuses, with an incidence of about 1 in 35,000 births and is due to twin-to-twin transfusion syndrome, frequently occurring in monochorionic twin pregnancies. The severity of the syndrome depends upon the type of the anastomoses arising between the vascular networks of the two fetuses, usually configuring the "twin reverse arterial perfusion syndrome". Case presentation: We report a case of monochorionic twins, one being an acephalus acardius amorphus fetus, showing features of extremely severe sistemic immaturity with no structured organs. Defined external and gross examinations have been performed on the dismorphic twin and placenta, along with the histological examination of tissue samples. The analysis of the placenta showed the vascular anomalies leading to the twin-totwin transfusion syndrome. The histopathological examination showed a plethora of findings from immature tissue with distorted architecture to peculiar histological structures such as blood vessels lined by melanocytic like cells. Conclusion: Monochorionic twins present high risk of complications, ranging from premature delivery to fetal death. The causative event of the acardia acephalus is controversial as divergent hypotheses have been pointed out, such as primary cardiac dysmorphogenesis versus hemodynamical mechanism, based on the abnormal vascular communication between embryos resulting secondary atrophy of the heart and dependent organs. In the case here reported we observed gross dysmorphic features in the malformed twin and extensive vascular anastomoses in the fused placenta, suggesting that the low pressure and hypoxigenated blood which nourished it could explain the extremely severe dismorphic sequence.

Keywords: acephalus Acardia, Twin reverse arterial perfusion syndrome, Monochorionic twins

\section{Anatomical and Angiographic Aspects of the Middle Meningeal Artery}

Obreja S Daria Alexandra', Oanță D Natalia Veronica', Orbu G Ana-Maria', Lecturer Dr. Razvan Stanciulescu'

"Carol Davila" University of Medicine and Pharmacy, Bucharest, Romania

Background: The purpose of this study is to present the anatomical and angiographic aspects of the middle meningeal artery, from the perspective of the endovascular and neurosurgical approach of the corresponding pathology. Materials and methods: The work has been carried out based on the dissection of the cephalic region of two adult formolized cadavers within the Anatomy Department of the University of Medicine and Pharmacy "Carol Davila" of Bucharest and by studying the database of the Interventional Radiology Laboratory of the Neurology and Cerebrovascular Diseases Institute of Bucharest. Results: By photographing the dissection stages we were able to expose in detail the topography of the temporal region, the pathway, the anatomical relations and the branches of the middle meningeal artery, and by studying the angiographic prcedures we have obtained indicative images for the anatomical variability and the clinical implications of this artery. Also, we obtain a series of comments that integrate the anatomical data with the clinical concepts. Conclusion: The close observation of the anatomical structures, vascular in particular, is an essential aspect in the understanding of the pathological aspects and constitutes a first step for the successful management of therapeutic solutions.

Keywords: middle meningeal artery, temporal region

26. Appendicular Mucocele - the Vaginal Way 
Botez C Andreea-Cristina', Zaharia G Marius-Cristian', MD PhD Ovidiu Nicodin ${ }^{2}$

"Carol Davila" University of Medicine and Pharmacy, Bucharest, Romania, " Gynecology Department, "Dr. Carol Davila" Central Military Emergency University Hospital, Bucharest, Romania

Background: Appendiceal mucocele (AM) is a rare pathology and is characterized by accumulation of mucin in the appendiceal lumen. Clinically, it can manifest with abdominal pain in the presence of a lower abdominal palpable mass. It is usually discovered intraoperatively. Proper preoperative diagnosis is mandatory since it requires surgical treatment and it must always be extracted intact. Effraction of AM could lead to development of pseudomyxoma peritonei, a pathology with a severe prognosis. Differential diagnosis with an adnexal mass is essential since an ovarian benign pathology could be extracted using the vaginal approach in selected cases. If the abdominal mass is an AM, then laparotomy or laparoscopy is elective. Case presentation: We present the case of a patient with persistent pelvic pain, most likely due to an ovarian cystic mass associated with uterine myoma. Taking into consideration ultrasound, uterine mobility, wide vaginal access, benign cervical and endometrial cytology, we offered the patient a vaginal hysterectomy since AM was not a diagnosis to consider at the time. Preoperative diagnosis of AM was impossible due to nonspecific ultrasound in the presence of gynecologic symptomatology and lack of more specific preoperative imaging. The major risk in the case of vaginal approach of such large probable ovarian mass, in reality an appendicular one, was mucocele effraction with subsequent development of pseudomyxoma peritonei (a pathology with a severe prognosis). The right anexial mass turned out to be an appendicular ovalar mass with tensed thin walls, adherent to a normal ovary, suggestive for appendicular mucocele. The appendicular mass was extracted intact, without effraction of the capsule. Pathology confirmed intraoperative diagnosis, establishing the simple mucocele form. Conclusion: Appendicular mucocele is a considerable differential diagnosis in cases of cystic ovarian mass. Proper preoperative diagnosis is important in establishing the surgical approach, in order to reduce the possibility of conversion to laparotomy, the risk of effraction of AM when using the vaginal route being considerable.

Keywords: appendicular mucocele, ovarian mass, vaginal, hysterectomy, pseudomixoma peritonei

\section{Invasive Adenocarcinoma of the Colon}

Vîlceanu C Irina Andreea', Robert-Mihai I.L Enache1, Iordache A Andrei1, Ionescu C Ruxandra Florentina', Lecturer Mihai Ciocîrlan',2, MD PhD Al Aloul Adnan³

"Carol Davila" University of Medicine and Pharmacy, Bucharest, Romania, "Prof. Dr. Agrippa Ionescu" Clinical and Emergency Hospital, Bucharest, Romania; , Department of Gastroenterology and Hepatology, 3"Prof. Dr. Constantin Angelescu" Clinical Hospital, General Surgery, Bucharest, Romania

Background: The colon adenocarcinoma is a malignant epithelial tumor that spreads beyond the mucosa, invading the deeper layers. In $60-70 \%$ of the cases, this cancer is presented in the sigmoid colon and the rectum. This pathology is a major cause of morbidity and mortality in the world, particularly in areas with increased socio-economic standards, affecting 1 of 20 persons. Sex related, it is the third most common type of cancer in both men and women. Case presentation: A 74 years old female patient with no significant medical history presents to the general practitioner for abdominal pain, alternating diarrhea and constipation, fatigue, slight weight loss, despite a good appetite in the last 2-3 weeks. Clinically, the patient is in a good general condition, the body mass index is 29 , the abdomen volume is increased through fat, mobile, soft, with no tenderness and the digital rectal examination (PR) is negative. Paraclinical investigations reveal mild iron deficiency anemia (hemoglobin $=10,4 \mathrm{~g} / \mathrm{dl}$ ), but nothing significant is visualized through abdominal ultrasound. Due to the anemia, it was considered mandatory to perform a colonoscopy that showed a vegetative tumor, slightly bleeding at $90 \mathrm{~cm}$ of the anocutaneous line, in the transverse colon. The biopsy fraction taken was insufficient, the interpretation describing only epithelial dysplasia. A computer tomography scan was performed that confirmed the tumor and there was no evidence of distant metastasis. The patient was operated on with curative intention- a segmental colectomy of the transverse colon and mesocolon. The histopathological diagnosis is tubular adenocarcinoma $\left(\mathrm{G}_{1}\right)$ that invades the muscular, serous, tubuloserous tissue and the areas of fat near the lymph nodes $\left(\mathrm{pT}_{3} \mathrm{~N}_{1} \mathrm{c}\right)$. Oncological treatment has been initiated for 6 months with chemotherapeutic agents (5Fluorouracil). Following re-evaluations over 2 years until now, the patient had a favorable evolution with complete remission confirmed by computer tomography scan and colonoscopy. Conclusion: We choose to present this case to raise awareness that iron-deficiency anemia with gastrointestinal transit disorders and weight loss over a short period of time are possible signs of a colon adenocarcinoma that require obligatorily an immediate colonoscopy.

Keywords: iron-deficiency anemia, adenocarcinoma, colonoscopy

\section{Metabolic Syndrome and obstructive Sleep Apnea Requiring Multidisciplinary Management}

Tătaru C Radu-Cristian', Țugui A Denisa-oana', Irina D Eusebiu Petru', Loghinoaia M Luciana-Maria', Assistant Lecturer Mădălina-Ioana Chiorescu², Associate Professor Laura Ciobanu², Professor Florin Mitu ${ }^{2}$

"Grigore T. Popa" University of Medicine and Pharmacy, lași, Romania, ${ }^{2}$ Medical Recovery Hospital, Iași, Romania

Background: The hallmark of obstructive sleep apnea (OSA) is the repetitive collapse of the upper respiratory tract during sleep, causing hypopneic-apneic episodes, sleep fragmentation. The imbalance between the sympathetic and parasympathetic nervous systems, the activation of the renin-angiotensin-aldosterone system, along with the condition's frequent association with metabolic syndrome lead to an increased individual cardiovascular risk. Case presentation: A 53-year-old patient with multiple cardiovascular risk factors (obesity, dyslipidemia, grade III hypertension, previous smoker - 51 pack-years and a history of alcohol abuse), with positive family history of cardiovascular disorders, is admitted to the Cardiovascular Rehabilitation Clinic in Iaşi accusing dyspnea upon mild physical exertion, fatigue and daytime sleepiness. Our clinical examination revealed a morbidly obese patient (BMI 50.5, abdominal circumference $145 \mathrm{~cm}$, Acanthosis nigricans), normal heart rate and blood pressure (BP) values under triple antihypertensive therapy $(130 / 85 \mathrm{mmHg})$. Routine blood tests showed hyperglycemia (113 mg\%), mildly elevated GGT levels and proteinuria (30mg/dl), optimal LDL values under lipid-lowering therapy (LDL-cholesterol $59.6 \mathrm{mg} \%$ ). The ECC revealed sinus rhythm $64 \mathrm{bpm}, \mathrm{QRS}$ axis -20 degrees, QS pattern in DIII, aVF. Echocardiography showed a preserved left ventricular ejection fraction ( $60 \%$ ) with no signs of diastolic dysfunction and normal kinetics. We performed a 24-h Ambulatory Blood Pressure Monitoring and a 24 -hour Holter ECG revealing optimal BP control (meanBP $125 / 77 \mathrm{mmHg}$ ), and no significant ventricular or supraventricular arrhythmias. We performed a cardiorespiratory polygraphy which diagnosed a moderate form of obstructive sleep apnea (apnea-hypopnea index 29/h, medium 02 saturation 91\%) requiring continuous positive airway pressure (CPAP) therapy. The cardiopulmonary effort test showed a severely reduced exercise capacity (Weber Class D). An abdominal ultrasound showed hepatic steatosis and mild pancreatic lipomatosis. Conclusion: The prevalence of obstructive sleep apnea is rising, partly due to the ongoing global obesity epidemic, thus representing another major cause of cardiovascular comorbidities. Our patient requires a multidisciplinary management, consisting of long-term CPAP treatment and the enrollment in a cardiovascular rehabilitation program (regular physical exercise, nutritional counseling, medical education and psychological support, control of risk factors). 
Screening for obstructive sleep apnea is advisable in all obese patients entering a cardiovascular rehabilitation program.

Keywords: metabolic syndrome, obesity, obstructive sleep apnea

29. Pituitary Adenoma and Its Implications in the Clinical Manifestations of Acromegaly

Soare S.D. Alexandru', Coauthors: Puscariu F Paul',2, Assistant Lecturer Homentcovschi Corina-Simona', MD Naidin Oana ${ }^{3}$

"Coltea" Clinical Hospital, Bucharest, Romania, "CCarol Davila" University of Medicine and Pharmacy, Bucharest, Romania, 3"Carol Davila" Nephrology Hospital, Bucharest, Romania

Background: We were very impressed by the complexity of this case, and so we tried to figure a way to show the world how a complete clinical tableau of acromegaly caused by pituitary adenoma looks like. Case presentation: Acromegaly is a disorder which is the result of hypersecretion of the Growth Hormone (CH) which occurs approximately in 3-4 people per 1 mil per year. Both males and females appear to be equally affected with an average age of presentation of 44 yo. (younger patients may have more aggressive tumors and higher $\mathrm{GH}$ concentrations). The cause of acromegaly is usually the pituitary adenoma,which is a benign tumor. The first symptoms include :enlargement of hands and feet,craniofacial dysmorphia, visceromegaly, type 2 diabetes, arterial hypertension. Also in the more advanced stages of the disease constipation, diverticulitis and megacolon, which induces a very high chance of colon cancer, may occur. In some cases, depending on the size of the adenoma, there may be a level of compression of the optic chiasma which can induce vision impairment. 74 yo.male, diagnosed 12 years ago with: type 2 diabetes, arterial hypertension, ischemic coronary disease and chronic constipation. Also, the patient presents a level of sensitive neuropathy and microvascular complications. After the clinical exam, a considerable growth of the limbs, massive hyperglossia, modified face structure and parotid hypertrophy had been discovered. After conducting an MRI scan, the following have been revealed: demyelinating as a result of ischemic microangiopathy and right-lateral pituitary modification with cranial hyperostosis, both compatible with pituitary adenoma. Also, by measuring the blood IGF1 (insulin growth factor) levels, which was found to be three times the normal level $(644.6 \mathrm{ng} / \mathrm{mL})$, directly related to the type 2 diabetes, the disease was further confirmed. Regarding the patient's chronic constipation a colonoscopy test has been conducted, which revealed that the patient was suffering of megacolon and diverticulitis. Conclusion: So what makes this case worth presenting? The fact that the diagnose of the adenoma was done in the a late stages of the disease, paradoxically overlaps with the polyposis which is an impediment in the surgical removal of the pituitary adenoma in the first place.

Keywords: pituitary adenoma, acromegaly, limbs enlargement, macroglosy

Severe Cutaneous and Systemic Reaction Related to Carbamazepine

Magdaș A Tudor-Mihai', Simionescu T Luca', Assistant Lecturer Bianca Simionescu

I"Iuliu Hațieganu" University of Medicine and Pharmacy, ClujNapoca, Romania

Background: Carbamazepine is prescribed not only as an antiepileptic drug, but also for other diseases as depression or bipolar disorder. In some predisposed individuals, there are possible severe cutaneous drug reactions, namely Stevens-Johnson Syndrome (SIS)/ Toxic Epidermal Necrolysis (TEN) associated with Carbamazepine. Case presentation: In the present work, a case of a 14 years old adolescent, medicated with Carbamazepine for two weeks, as a mood stabilizer, is reported. A systemic reaction with flu-like symptoms, high fever, severe oral erosions, conjunctivitis, and a generalized cutaneous, initially erythematous painful eruption, then extended epidermal detachment, appeared. The diagnosis of TEN was made and the skin biopsy confirmed the clinical diagnosis. The laboratory findings were normal. Carbamazepine was immediately discontinued. Supportive measures, intravenous fluids, wound care, intravenous immunoglobulin, and a course of intravenous cortisone hemi succinate, then oral prednisolone with progressive tapering were introduced. The clinical resolution appeared progressively within two weeks, but with squeals as skin depigmentation, nails dystrophy and anxiety. Conclusion: The physicians should be aware of the potential role of high-risk medication in triggering SJS or TEN. The early diagnosis and specific management is mandatory of to prevent or limit long-term sequelae.

Keywords: carbamazepine, Stevens Johnson Syndrome, toxic epidermal necrolysis, adolescent

\section{Significance of Multidisciplinary Team in Treating Metastatic Colon Cancer \\ Munkena I Zane', MD Evita Gasenko²}

'University of Latvia, Riga East University Hospital, Department of Chemotherapy and Haematology, Riga, Latvia, ZUniversity of Latvia, Riga, Latvia

Background: Clinical case study describes a 38 years young patient with metastatic colon cancer. Multidisciplinary approach in the patient's evaluation and combination of local and systemic treatment methods achieved a full remission. Patient's overall survival now exceeds 2.5 years and the quality of life is preserved. Case presentation: Patient: 38-year-old woman complained about liquid stool and diffuse pain in the abdomen for 2 years. In January 2015 patient had an episode of severe abdominal pain in the hypogastric region. Colonoscopy showed an uneven mass in the area of the hepatic angle of colon ascendens covering $2 / 3$ of the colon lumen. Histologically: moderately differentiated adenocarcinoma of the colon. CT scans of the abdominal cavity showed metastasis in $\mathrm{Sg} 7$ of the liver. Multidisciplinary board made a decision to start treatment with a surgery. Right hemicolectomy, extended lymphadenectomy and atypical resections of Sg2,3,7 of the liver were performed in March 2015. Final clinical diagnosis was: $\mathrm{Ca}$ colon ascendens $\mathrm{T}_{3} \mathrm{~N}_{1} \mathrm{bM}_{1} \mathrm{C}_{2} \mathrm{Ro}$ Stage IV. Histological material was tested for RAS mutation. Mutation was detected in $12 \mathrm{AGT}$ and $\mathrm{A} 146 \mathrm{X}$ genes. After surgery patient received 12 courses of FOLFOX IV chemotherapy. When therapy was completed CT examinations (October 2015) showed a complete remission. Follow-up time CT examination performed in August 2016 revealed 3 intraparenchymal metastases in Sg2,4,7 of the liver. Left hemihepatectomy was performed. During the hospitalisation patient had complaints about severe pain and discomfort in the right hypochondrium and MRI revealed thrombosis of the medium and right veins in the liver with secondary extensive ischaemia of the right lobe and a postoperative biloma in the left lobe of the liver. Patient received anticoagulant and antibacterial therapy which improved patient's state. From November 2016 to April 2017 the patient received 12 courses of FOLFOX IV chemotherapy and anticoagulant therapy with Rivaroxabanum $20 \mathrm{mg}$. After therapy was completed CT examinations showed complete remission and the region of the ischaemia in the liver decreased in size. Conclusion: The clinical case shows the significance of multidisciplinary team in treating metastatic colorectal cancer patients, early diagnostics and correction of treatment complications, and the importance of local therapeutic approaches in prolonging overall survival.

Key words: metastatic colon cancer, multidisciplinary care

\section{The Effects of Sleep Deprivation on Future Doctors}


Szatan G Dalma-Zsuzsa', Tarta N Amalia', Lavinia-Maria G Tusan', Lecturer Mekeres Florica'

University of Oradea, Faculty of Medicine and Pharmacy, Oradea, Romania

Background: Sleep deprivation, the state of not sleeping enough. It can be acute or chronic. A chronic state of sleep deprivation can provoke tiredness, daytime somnolence, hunger as well as weight gain or loss. With this study we wanted to observe the effect of acute sleep deprivation on medical students. We studied which side effects show first and how the tested skills deteriorate with the more time spent without sleep. Materials and methods: We put 10 medical students to the test with the ages between 19 and 23. We analyzed how the lack of sleep affects the following skills: memory, communication, logical thinking, reflexes, concentration and motoric precision. This was possible through a test we put together, that contained exercises and tasks through which we were able to test the skills mentioned above. Each of the subjects got 3 envelopes and a sheet with instructions. The envelopes contained a set of tasks with decreasing difficulty in each envelope. The subjects had to complete them after 10, 20 and 30 hours spent consecutively without sleeping. Results: The results show that besides not falling asleep, the most difficult task was the origami, through which we tested concentration and motoric precision. The students had also had difficulties with following instructions and solving logic puzzles. None of the students could complete the task without any sleep, $60 \%$ of the students had problems with memorizing tasks. The testing of the reflexes and the short term memory showed decreasing results. An interesting observation was that the motoric precision, which is a skill that along with the ability of following instructions are often used by medical students completing practical tasks, was extremely deteriorated by sleep deprivation. This issue can lead to severe mishappenings in the field of surgery. Conclusion: Our study proved that a rested mind and body works better, solves problems quicker, thinks faster, communicates more efficiently and moves more precisely. In the case of an acute sleep deprivation the set of skills that are needed by a medical student gets deteriorated, which explains, for example, why more rested students get better grades on exams then their sleep deprived colleagues.

Keywords: sleep deprivation, lack of sleep, concentration, memorizing, logical thinking, short term memory, sleep deprivation effects

\section{The Percutaneous Device Closure of Ventricular Septal Defects}

Cristescu C Miruna', Baluta S Iulia', Dumitrescu C.I. Denisa Monica', Assistant Lecturer Bogdan Ursut ${ }^{1,2}$

"Carol Davila" University of Medicine and Pharmacy, Bucharest, Romania, 2"Prof. Dr. Agrippa Ionescu" Clinical and Emergency Hospital , Bucharest, Romania

Background: The ventricular septal defect is one of the most common congenital malformations of the heart, accounting for up to $40 \%$ of all cardiac anomalies. Taking into consideration the anatomical varabilities, the age of the patients or associated malformations the therapeutical approach becomes difficult. Traditional open heart surgery was used for a long time, but being associated with complications, alternative techniques have been developed. Transcatheter closure is less inasive and reduces the risk of postoperative afflictions. Materials and methods: The percutaneous device closure requires introducing a catheter along the femoral artera until it reaches the heart. The defect is crossed with the guide wire from the left ventricle aspect to create an arteriovenous circuit. Then, the wire is exteriorised either to the jugular or femoral vein. The device is introduced through transvenous approach and attached to both sides of the septum, under transesophageal echocardiographic guidance. The expansion of the device occludes the passage and restricts the abnormal blood flow. The intervention is performed under general anaesthesia and does not require cardiopulmonary bypass.
Results: The defect is completely occlused. The residual shunt is reduced or absent. Pulmonary tension decreases to normal values. The method is feasible and effective and is not associated with severe complications. The recovery time is shorter compared to the conventional surgical treatment and the hospital stay is reduced. Estetically, the remaining scar is minor determining a smaller psychological impact. Conclusion: Although controversed for a long time, the transcatheter closure of ventricular septal defects is more effective, with better outcome on either short or long term. It is minimally invasive and offers an important alternative to the surgical approach. Wherever possible, this novel form of therapy should be considered the treatment of first choice.

Keywords: ventricular septal defect, percutaneous closure, device, alternative

\section{Unusual Case of Locally Advanced Cervical Cancer Treated} Using Radiotherapy and Chemotherapy Complicated with Gastric and Hepatic Metastases

Doriana-llinca D Volanschi', M Diana Elena', Ciobanu S Andreea-Roxana', Florea C Cristina-Maria', MD Huțanu Ionuț ${ }^{1,2}$

"Grigore T. Popa" University of Medicine and Pharmacy, lași, Romania, ${ }^{2}$ Regional Oncology Institute, Iassi, Romania

Background: Cervical cancer remains a major cause of mortality in Romania, in spite of numerous screening programmes designed for the prevention and early diagnosis of the disease. In terms of etiology, the sexually transmitted Human Papilloma Virus infection is most commonly associated with the type of cancer. The diagnosis is confirmed either through the Papanicolau test or colposcopy with biopsy sampling. Case presentation: 45 year old female patient was admitted to the Regional Institute of Oncology Iaşi with cervical cancer (invading the bladder and pelvis) diagnosed in 2014, symptomatically manifested through menometrorrhagia and pelvic pain. The anatomopathological examination stated that the neoplasm was an in situ microinvasive squamous cell carcinoma and the RMI showed that it was a stage IVa $\mathrm{M}_{1}$ cancer. The patient underwent neoadjuvant chemotherapy and radiotherapy, after which there was no evidence of remaining tumoral cells. In 2015 the patient was diagnosed with antral gastric tumor and in 2016 during CT a voluminous expansive hepatic mass wass identified in segments 7 and 8. Later on, these proved out to be metastases originating from the squamous cell carcinoma in the cervix. For the gastric tumor the surgical approach was a subtotal gastrectomy with Roux-en-Y gastro-jejunal anastomosis. During a following intervention, a tumoral mass of umbilical aspect with a $5 \mathrm{~cm}$ capsular expresion and another $2 \mathrm{~cm}$ nodule located in the 6 th segment were identified in the liver. Hepatic tumorectomy was performed, but after one year the patient developed yet another metastasis in the liver. Conclusion: The particularity of the case consists of the rare topography of the metastases, given the fact that cervical cancer generally spreads in the pelvis and lymphatic nodes. Furthermore, what makes it even more intriguing is the fact that the local response to radiotherapy was very good with no signs of remaining tumoral cells in the cervix.

Keywords: cervical cancer, gastric metastases, hepatic metastases, iradiation, squamous cell carcinoma

35. Utility of Diuretic Time in Radionuclide Renal Investigation Irina I Vizureanu', Professor Cipriana Ştefănescu', Lecturer Irena Cristina Grierosu

" Grigore T. Popa" University of Medicine and Pharmacy, Iași, Romania

Background: Renal disease prevalence in children has been increasing in the last ten years. The principal causes are congenital hydronephrosis due to pielo-ureteral stenosis, primary 
megaureter and vesicoureteral reflux. Case presentation: We show the clinical case of an 8 years old patient who was diagnosed in 2015 with vesicoureteral reflux, grade III, with a clinical history of recurrent urinary tract infections. He was referred to our nuclear medicine department to quantify the renal function. The images were taken immediately after radiotracer administration (99mTcDTPA) with a dual-head gamma camera Siemens with diuretic time administration at 20 minutes. In 2016, the follow-up nephrography was performed under the same technical conditions, with the sole exception of diuretic time infusion, being changed from 20 minutes to the first minute after radiotracer injection. Conclusion: First nephrography was made immediately after diagnosis. The nephrographic curves showed a normal pattern of uptake and elimination for the left kidney while for the right kidney, the curve pattern was equivocal. After one year, during the follow-up examination, the diuretic time was changed to be infused in the first minute. The nephrographic curves had a more specific pattern: the left kidney function was unaltered as in baseline exploration and the equivocal image for the right kidney was normalized, meaning there was only a dilatation. The diagnosis between renal collecting system obstruction and dilatation has direct implications in the subsequent therapeutic attitude. We considered that the diuretic protocol in first minute for the nephrographic investigation of hydronephrosis significantly reduces the equivocal cases.

Keywords: renal disease, nephrographic, hydronephrosis, diuretic time, equivocal case

\section{Volume-Staged Radiosurgery for High-Grade}

Scriminti I Diana Gabriela', C.C Raul Alexandru', MD PhD Constantin Tuleasca ${ }^{2,3}$, MD PhD Iulia Peciu-Florianu' ${ }^{2,4}$, Professor Marc Levivier ${ }^{2,3}$

"'Grigore T. Popa" University of Medicine and Pharmacy, Iasi, Romania, ${ }^{2}$ Neurosurgery Service and Gamma knife Center, Lausanne University Hospital, Switzerland, 3University of Lausanne, Faculty of Biology and Medicine, Lausanne, Switzerland, 4RogerSalengro Hospital, Lille, France

Background: The current management of high-grade arteriovenous malformations (AVMs) involves a coordinated multimodal treatment, combining observation, microsurgery, endovascular and radiosurgery (RS). In these complex cases, volume-staged RS can be used to decrease complications risks and provides still high obliteration rates. We prospectively analyze the safety and efficacy of Gamma Knife surgery (GKS) in 6 challenging cases. Materials and methods: Six patients with Spetzler-Martin grade III (one), IV (3), and V (2) AVMs were treated in Lausanne University Hospital between 2010 and 2015. One patient had previous surgery for hematoma evacuation while another two benefitted from prior endovascular embolization. Three received GKS as the first treatment option. All patients were followed at 6 and 12 months and then on a yearly basis with serial brain MRI and angiography (after MRI showed complete obliteration). Results: Mean age was 40.8 years (range 28-60). Initial clinical presentation was hemorrhage (two) and epilepsy (three). Mean follow-up 38.4 months (range 12-48). For stage 1: mean target volume (TV) was 182.4 CC (range 45-555), mean prescription isodose volume (PIV) of $250.8 \mathrm{cc}$ (range 55-673), volume of the $12 \mathrm{~Gy}$ isodose line for stage $117.7 \mathrm{Cc}$ (range 5.75-27), the mean marginal dose was $19.8 \mathrm{~Gy}$ (range 18-20). The mean period between the two stages was 6.6 months (range 6-9). Only one patient had an increase in seizure frequency during this period. No hemorrhagic events were noted. For stage 2, mean TV GKS was $172.4 \mathrm{cc}$ (range 22-474), mean PIV of 275.6 CC (range 6-673), mean marginal dose was 19.6 Gy (range 1820), volume of the $12 \mathrm{~Gy}$ isodose line was $13.85 \mathrm{Cc}$ (range 6.7-21). Radiological assessment at last follow-up showed partial obliteration in all cases, with digital subtraction angiography confirmation in 1 . Only one patient required microsurgical intervention at follow-up, for the residual nidus. Conclusion: Volume-staged radiosurgery is a safe and effective treatment for high-grade AVMs, providing higher rates of obliteration without an increase in possible complications. In these complex cases GKS can be successfully combined with surgical or endovascular approaches, for an optimal multimodal treatment.

Keywords: Gamma Knife surgery, high-grade arteriovenous malformations (AVMs), Volume-staged radiosurgery

\section{Wolff-Parkinson-White Syndrome Treatment and Diagnosis Difficulties.}

Țîrlea M.S. Stefan Alexandru', Macsim C Mihai Andrei', Podgoreanu M Bogdan', Stan M Anca', Assistant Lecturer Remus Mihalcea ${ }^{2}$

1"Carol Davila" University of Medicine and Pharmacy, Bucharest, Romania, '2County Emergency Hospital, Pitești, Romania

Background: Wolff-Parkinson-White syndrome is a rare congenital disorder in which an extra electrical pathway between the atria and ventricles is present, which bypasses the Atrio-Ventricular Node. Episodes of fast heartbeats can occur but aren not life threatening on their own. This disorder affects only $0.2 \%$ of the population. Complications appear when other heart anomalies are present thus Wolff-Parkinson-White syndrome must be treated either using radio-frequency catheter ablation or medication. Case presentation: Ms D.S. is a 52 years old secretary from Cluj-Napoca who was hospitalized on 14.03 .2015 with complaints of high frequency rhythmic palpitations and retro-sternal pain. In 2013 she was diagnosed with Ebstein's anomaly and Wolff-Parkinson-White syndrome with episodes of atrial fibrillation and type 2 hypertension associated with obesity. The appropriate treatment at the time was ablation with radio-frequency catheter of the postero-septal wall. After a clinical exam, we can see the tegument and mucous have physiological aspect and no leg swelling is observed, normally conformed thorax, rhythmic heart noises with a heart rate of 60 BPM (beats per minute) and a blood pressure of $140 / 80 \mathrm{mmHg}$. Further exams reported cardiac failure type NYHA 2 (New York Heart Association Classification Criteria) mitral insufficiency based on the deceleration of the systolic murmur in the mitral area and tricuspid insufficiency and relapse of the ablation 2 weeks after the procedure. After performing 4 EKG (electrocardiogram) exams, results showed the PR wave length < $120 \mathrm{~ms}$, QRS complex length > $120 \mathrm{~ms}$ with delta wave present both at the debut and the end of the QRS complex. Following the EKG results, the most plausible output was the diagnosis with Wolff-Parkinson-White Syndrome. Further complications should be reported as following: 1. Relapse risk of the second ablation; 2 . The general perspective shows death risk of $19.5 \%$ considering all the factors previously mentioned; $3.17 .1 \%$ risk of worsening to cardiac insufficiency NYHA III or IV. Conclusion: This case is highlighted by both the association of two rare congenital diseases (The Wolff-Parkinson-White syndrome and the Ebstein's anomaly) and diseases provoked by internal and environmental factors (obesity, stress). The way these combined factors react explained the need of writing this case report.

Keywords: Wolff-Parkinson-White syndrome, Ebstein's anomaly, systolic murmur, ablation with radiofrequency

\section{Amyloid-Beta Peptide is Required for the cGMP-Induced Long-Term Potentiation and Memory \\ Tropea - Maria Rosaria', MD PhD Daniela Puzzo' \\ 'Department of Biomedical and Biotechnological sciences, University of Catania, Catania, Italy}

Background: Accumulation of amyloid-beta $(A \beta)$ has been related to Alzheimer's disease pathogenesis. However, in the healthy brain, low concentrations of $A B$ are necessary for physiological long-term potentiation (LTP) and memory. Because cGMP plays a key role in these processes, here we have investigated whether CGMP might influence $A \beta$ production and function during LTP and memory in physiological conditions. Materials and methods: We first evaluated whether an increase of CGMP levels by 
phosphodiesterase-5 inhibitors (PDE5-Is) might affect A $\beta$ levels in Neuro-2a ( $\mathrm{N} 2 \mathrm{a}$ ) cells and hippocampal slices. We also evaluated whether PDE5-Is might modify Amyloid Precursor Protein (APP) expression and the interaction between APP and the $\beta$-site APP cleaving enzyme-1 (BACE-1), measured with the OptiCAB assay. Finally, we performed electrophysiological experiments on hippocampal slices and behavioral studies (novel object recognition) to analyze whether the PDE5-Is-induced enhancement of LTP and memory was still present when blocking A $\beta$ function. Results: We showed that the increase of intracellular CGMP after a treatment with PDE5-Is induced a parallel increase of $A \beta$ levels in N2a cells and hippocampal slices. This effect was reduced by the guanylyl cyclase inhibitor ODQ. PDE5-Is did not modify APP fulllength expression but increases the APP/BACE-1 convergence in endolysosomal compartments. Finally, we demonstrated that the CGMP-induced LTP and memory depended upon A $\beta$ production. In fact, the physiological potentiation of LTP and recognition memory induced by PDE5-IS was not present if blocking $A \beta$ function - by anti-murine A $\beta$ antibodies or APP knock-out mice. Conclusion: The increase of CGMP positively modulates $A \beta$ production, which, in turn, boosts synaptic plasticity and memory. The lack of effect of PDE5-Is in APP KO mice suggests that A $\beta$ is needed for the CGMPinduced enhancement of LTP and memory. Thus, PDE5-Is might work as cognitive enhancers via a positive modulation of $A B$ at physiological concentrations in the brain.

Keywords: APP, amyloid beta, CGMP, long-term potentiation, memory, synaptic plasticity

\section{Beneficial Supplement Mixture for Fatty Liver}

Mitran G.S Raluca-Elena', MD Mihaela Balaban', MD Adriana Dinu', Lecturer Daniela Miricescu', Associate Professor Alexandra Totan', Professor Maria Mohora', Associate Professor Bogdana Virgolici'

'"Carol Davila" University of Medicine and Pharmacy, Bucharest, Romania

Background: A hypercaloric and hyperlipidemic diet induces nonalcoholic fatty liver disease (NAFLD), which is frequently associated with type 2 diabetes mellitus, metabolic syndrome, obesity and dyslipidemia. Anti-inflammatory and antioxidant supplements may prevent or reverse NAFLD. Vitamin $E$ and fish oil have these properties. Poly-lactic-co-glycolic acid (PLCA) nanoparticles charged with vitamin $\mathrm{E}$ have liver tropism and increase the water solubility of the vitamin. The aim of the study is to investigate the effect of the combination of PLGA vitamin E and fish oil on fatty liver. Materials and methods: Twenty male Wistar rats, 3 months old, were fed with a hypercaloric and hyperlipidemic diet for 4 weeks. Then, for the next 3 weeks they were fed with a standard diet, but they were divided into 4 groups as follows: group 0 was only on a standard diet, group $\mathrm{N}$ was treated with PLCA nanoparticles with vitamin $E(1 \mathrm{mg} / \mathrm{kg})$, group $L$ took Lysi fish oil $(1 \mathrm{mg} / \mathrm{kg})$ and group $M$ received both PLCA nanoparticles with vitamin $E$ and Lysi fish oil, same doses. Blood samples were taken for glucose, triglycerides, total proteins, cholesterol, alanine aminotransferase (ALT), cystatin, gamma glutamyl transferase (GGT), alkaline phosphatase (ALP), urea and creatinine measurements and liver oxidative stress markers (malonyldialdehyde-MDA, glutathione, thiols) were determined. Liver was harvested and stored in a $10 \%$ solution of formaldehyde (formol) for histopathological examination. Results: Group 0 had the worst systemic biological profile. Compared to group 0 , group $\mathrm{N}$ showed significantly decreased $(p<0.05)$ levels for all hepatic enzymes, reduced $(p<0.05)$ cholesterol and cystatin. Group L showed significantly decreased levels of triglycerides $(p=0.0002)$, ALT $(p<0.0191)$, cholesterol $(p=0.0003)$, cystatin $(p<0.0001)$ and GGT $(p=0.0082)$. Group $M$ showed significantly decreased ( $p<0.015)$ levels of triglycerides and hepatic enzymes activity. Regarding oxidative stress markers, group $M$ showed $(p=0.0387)$ the lowest levels for MDA and the highest $(p=0.0013)$ for glutathione. At the histopathological exam, group 0 showed medium hepatopathy, while groups $\mathrm{N}, \mathrm{L}$ and $\mathrm{M}$ showed only minor hepatopathy. Groups L and M showed a decreased number of inflammatory cells at the histopathological exam. Conclusion: The combination of Lysi fish oil and PLGA nanoparticles with vitamin $E$ has synergistic hepatoprotective effect.

Keywords: PLGA nanoparticles, vitamin E, fish oil, oxidative stress, hepatopathy, dyslipidemia, metabolic syndrome

\section{Body Temperature Flux as an Early Detection Method of Breast Cancer}

Hovornyan Leonid Serhiy', Postevka Dmytrivna Iryna', Professor Ivashchuk Oleksandr ${ }^{1}$

'Bukovinian State Medical University, Chernivtsi, Ukraine

Background: Breast cancer continues to be the most widespread cancer among women. The most effective way to improve the results of treatment is screening methods improvement. This indicates to the need of creation and implement of new noninvasive technologies that would complement traditional early detection methods. An indicator of biological processes in the human body is the heat flux of internal organs, the magnitude of which is varies with different pathology, in particular oncologic processes. The aim of the study was to analyze experimentally and clinically the density of the temperature flux of the mammary gland in special that which was affected by gastric neoplasms. Materials and methods: The experiment was performed on 48 rats. The main group consisted of 32 animals, which were grafted with the Heren tumor into perineum tissues in project localization of mammary glands, according to the method we proposed (patent NN109812 dated 12.09.2016). And 16 intact animals created the comparison group. Thermal measurement was carried out from the 8 th to the 16 th days after the tumor injection. Clinical trials were performed on 125 women, who were divided into two groups. The main group was 37 women with breast cancer, in I-II A stage of the disease. A comparison group was created by 88 practically healthy women. A multichannel device ALTEK-10008, with the software "Thermologger 9004TS-M", carried out measurement of the heat flux in the mammary gland. Results: The results of the experimental study indicate that the heat flux in the mammary glands with tumor is lower than intact and decreases with the growth of the tumor. The results of the clinical trial indicate that there is no probable difference in the density of the heat flux during all stages of the menstrual period. The presence of malignant tumors of the mammary gland leads to a decrease in the density of the heat flux in the projection area in 1.21 times ( $p<0.01)$. Conclusion: Consequently, the density of the heat flux of the mammary gland completely reflects the morphofunctional state of its tissues, which can be used for the early detection of cancer in this localization.

Keywords: breast cancer, tumor, gastric neoplasms, early detection methods, mammary glands, temperature flux

\section{Can We Teach Auscultation in a Different Way? \\ Anghel I.C. Hrista Teodora', MD PhD Cristian Toma ${ }^{2}$ \\ "Carol Davila" University of Medicine and Pharmacy, Bucharest Romania, 2"Prof. Dr. Theodor Burghele" Clinical Hospital, Bucharest, Romania}

Background: Due to the continuously increasing number of students in our university, the opportunity of practicing clinical maneuvres on pacients, such as cardiac auscultation, becomes smaller and smaller. Moreover, a competent cardiac auscultation, combined with an optimal level of confidence and experience, is essential to every future doctor. Therefore, medical simulation is the perfect solution for improving these much needed clinical abilities, in a relaxing and supportive environment. Materials and methods: We evaluated the evolution of 59 students, from the 3 rd to the 6th year of study, who took part in 4 hands-on workshops of cardiac auscultation, in a training center. The duration of the 
workshops was 6 hours each. The presentation was held by a specialist doctor, who showed and explained them the tehnique, presented them the normal heart sounds and then more than 20 types of murmurs. After that, each student had the chance to perform the tehnique at least 2 times, while the others could also hear the cardiac sounds on a microphone. All of them were asked to complete a form, before and after the workshop, in order to evaluate themselves and the progression they have made. Furthermore, we called a number of 10 students who participated on the trainings and in the meantime started the medical residency and asked them for their opinion on the utility of the workshop. Results: The results have clearly shown a progress in each student's ability to correctly diagnose a cardiac pathology-on average, they autoevaluated it with 2 points out of 4 - as well as an increase in their self-confidence- 3 points- and interest in cardiology. Conclusion: In conclusion, in this era of astonishing technological advancement, the medical simulation offers a good alternative to the bedside teaching and represents a very efficient manner to develop the clinical abilities of future doctors.

Keywords: medical simulation training, international students, task-trainer simulators, medical education, cardiac auscultation

42. Cellular Viability Dynamics Due to Chloride Symporter Manipulation in Mature Hippocampal Cultures Exposed to Metabolic Injury

Ionescu C Rosana-Bristena', Mara², Chitimus Diana', Associate Professor Ana-Maria Zagrean ${ }^{2}$

"Carol Davila" University of Medicine and Pharmacy, Bucharest, Romania, 2Department of Physiology and Neuroscience,"Carol Davila" University of Medicine and Pharmacy, Bucharest, Romania

Background: Neurons exclusively depend on the aerobic breakdown of glucose, metabolic deprivation causing substantial viability impairment, materialized as a marked ionic imbalance. A major role in this mechanism is played by the $\mathrm{Cl}$ - ion whose cytoplasmic concentration appears to increase during metabolic injury episodes due to induced changes in the expression of $\mathrm{Cl}$ symporters $\mathrm{NKCC}_{1}$ and $\mathrm{KCC}_{2}$. By chemically manipulating the function of these symporters in an in vitro cerebral ischemia model we were able to assess the correlation between their expression, ionic concentration dynamics and neuronal viability. Materials and methods: Primary hippocampal neuron cultures were obtained from Wistar rats on postnatal day 0 . After 7 days in vitro (DIV7), mature cultures were exposed to $2 \mathrm{~h}$ of OGD (oxygenglucose deprivation) or control conditions (normoxia in the presence of a glucose-medium). Evaluation of cellular metabolism was performed using the resazurin viability test during a $3 \mathrm{~h}$ reoxygenation in a normoglycemic environment. Cell viability after exposure to OGD was compared to control-viability, considered as $100 \%$. NKCC1 antagonist bumetanide or KCC2 antagonist DIOA were added to part of the OGD-exposed wells either during the OGD or reoxygenation stages. Results: The exposure of mature hippocampal cultures to OGD+bumetanide conditions led to an increase in cellular viability, while the exposure to OGD+DIOA conditions resulted in a decrease in viability, both compared to OGD-exposed cultures. Neuronal cultures treated with bumetanide or DIOA during reoxygenation showed no signifficant differences in viability in comparison to OGD-exposed cultures. Conclusion: The potential neuroprotective effect of bumetanide might be due to an increase in the expression of NKCC 1 during ischemic episodes. By blocking the inward $\mathrm{Cl}$ - transport the bumetanide-treatment leads to a decrease in its intracellular concentration. In contrast, the detrimental effects of the DIOA-treatment may be explained by the supplementary increase in $\mathrm{Cl}$ - intracellular concentration due to the blockage of its outward transport. DIOA's lack of significant effect when added during the reoxygenation stage is most probably due to a marked decrease in $\mathrm{KCC} 2$ expression after ischemic exposure, whereas in the case of bumetanidereoxigenation-treatment the explanation resides in NKCC 1's change in transport dynamics due to modified intracellular $\mathrm{Na}+$ and $\mathrm{Cl}$ concentrations at the end of OGD-exposure.
Keywords: mature hippocampal neurons, oxygen and glucose deprivation, bumetanide, DIOA, ischemia

\section{Determination of VECFR-2 (KDR) 604A>C Polymorphism in Pancreatic Disorders}

Cîmpeanu D.R. Radu - Cristian', D Denisa-Maria', Dumitrascu J Andreea-Ramona ${ }^{2}$, Stoica M Mihaela Oana Alexandra', MD PhD Petrescu Florin³, Assistant Lecturer Pădureanu Vlad', Associate Professor Ioana Mihai ${ }^{4}$, MD PhD Săftoiu Adrian'

'Research Center of Gastroenterology and Hepatology, University of Medicine and Pharmacy, Craiova, Romania, 2Human Genomics Laboratory, University of Medicine and Pharmacy, Craiova, Romania, 3Discipline of Internal Medicine, University of Medicine and Pharmacy, Craiova, Romania, 4Discipline of Medical Genetics, Human Genetics Laboratory, University of Medicine and Pharmacy, Craiova, Romania

Background: Pancreatic diseases have a high prevalence worldwide. In spite of the fact that screening methods became more effective and the knowledge we have nowadays about pancreatic diseases has enhanced, their incidence remains high. Material and Methods: Our purpose was to determine whether single nucleotide polymorphism (SNP) of VEGFR-2/KDR (vascular endothelial growth factor receptor 2/ kinase insert domain receptor) influences susceptibility to develop pancreatic pathology. Genomic DNA was extracted from blood samples collected from patients diagnosed with acute pancreatitis $(n=111)$, chronic pancreatitis $(n=38)$, pancreatic cancer $(n=91)$ and healthy controls $(n=251)$. VEGFR-2 (KDR) 604A>C (rs2071559) polymorphism frequency was determined with TaqMan allelic discrimination assays. Results: Statistical assessment was performed by associating genetic polymorphism with clinical and pathological data. In both pancreatic diseases and healthy control groups the polymorphism we studied was in Hardy-Weinberg equilibrium. Association between increased risk for pancreatic diseases and studied polymorphism was statistically significant. KDR 604AG and AG + GG genotypes were more prevalent in acute pancreatitis and pancreatic cancer patients than in controls. These genotypes influence disease development in a low rate. No association was found between chronic pancreatitis and KDR 604AG and AG + GG genotypes. In Romanian cohort, we found an association between the $\mathrm{KDR} 604 \mathrm{~A} \rightarrow \mathrm{G}$ polymorphism and acute pancreatitis and pancreatic cancer. Conclusion: Carriers of the604G variant allele were more frequent among acute pancreatitis and pancreatic cancer than among controls, suggesting that KDR $604 \mathrm{G}$ allele may confer an increased risk for these diseases. In the future, more extensive studies on larger groups are necessary, in order to emphasize the role of VEGFR2 polymorphisms in pancreatic pathology.

Keywords: pancreatic disorders, polymorphism, genotype, VEGFR-2

\section{Effects of Gold Nanoparticles Functionalized with Albumin} on Pancreatic Adenocarcinoma Migration Capacity

Gonciar D Diana', Lecturer Teodora Mocan', MD PhD Tudor Cristian Matea², Lecturer Lucian Mocan

"Iuliu Hatjieganu" University of Medicine and Pharmacy, ClujNapoca, Romania, 'Regional Institute of Gastroenterology and Hepatology "Octavian Fodor", Cluj-Napoca, Romania

Background: Gold nanoparticles (GNPS) have proved their role as effective photothermal agents. Moreover, it has been emphasized that by Albumin (Alb) functionalization, the selectivity of thermal destruction is enhanced through the overexpression of Alb receptors in epithelial-derived cancer specimens. However, the effects of long-term administration of Alb-GNPs on cell migration is still uncertain. Materials and methods: Synthesis and functionalization of GNPS with human serum albumin was performed. Complete characterization of the newly designed 
nano-structures was achieved by FTIR, UV-VIS, AFM and Raman Spectroscopy. Panc-1 cell line was used for in vitro studies. Exposure to the nanomaterial was conducted using different concentrations of nanomaterial $(50,25,17.5 \mu \mathrm{g} / \mathrm{mL})$ and a special migration assay was used (Mililuapore) with flow cytometry evaluation. Results: Our results showed no significant morphological changes or migration capacity of Panc-1 cells after exposing them to 17.5 and $25 \mu \mathrm{g} / \mathrm{mL}$ Alb-GNPs. However, higher concentrations of nanomaterial $(50 \mu \mathrm{g} / \mathrm{mL})$ have the tendency to promote cell migration and may lead to metastasis formation. Conclusion: Even if GNPs are known to exhibit impactful features, our results demonstrate the need for reevaluation of long term administration of nanomaterials. A proper balance between the need for efficacy and the need for the reduction of pro-metastatic effects should be maintained.

Keywords: gold nanoparticles, albumin, migration, pancreatic adenocarcinoma

45. Experimental Investigations of Taurine Effects in Somatic and Visceral Nociceptive Reactivity in Mice

Damaschin D Ana', Damaschin D. Andrei', Associate Professor Liliana Mititelu-Tartau'

"Grigore T. Popa" University of Medicine and Pharmacy, Iași, Romania

Background: Taurine (2-aminoethane sulfonic acid) is a component of different food, being regularly present into energy drinks. Its synthesis in the human body is realized primarily in the liver, through the cysteine sulfonic acid pathway, with the participation of pyridoxal-5' phosphate. Directly or indirectly, taurine is capable to manifest either a confirmed or highly feasible involvement in a various pathological states, that disturb practically every main organs and is responsible for a wide variety of serious diseases. Materials and methods: The aim of our study was the experimental researches of the effects of taurine in somatic and visceral pain models. The experiment was carried out on white Swiss mice (20-25g), distributed into 3 groups of 7 animals each, treated intraperitoneally as following: Group I (Control)- saline solution $0,1 \mathrm{ml} / 10 \mathrm{~g}$ body weight (bw), Group 2 (TAU)- taurine 800 $\mathrm{mg} / \mathrm{kg}$ bw, Group 3 (IBU)- ibuprofen $5 \mathrm{mg} / \mathrm{kg}$ bw. The nociceptive cutaneous testing was performed using tail-flick model, to evaluate the latency period reaction after thermal noxious tail stimulation. The tail withdrawal latency (seconds) was counted before the experiment, and 15, 30, 60, 90 minutes after the substances administration. The model of visceral pain used, consists of chemical peritoneal irritation with acetic acid (0,6\%). Pain responses were scored by counting the number of stretches, or writhes per animal, every 5 minutes, during 30 minutes interval, in the experiment. Ibuprofen was used as positive control drug, with known analgesic effects in these experimental models in mice. The protocol was approved by the "Grigore T. Popa" University Committee for Research and Ethical Issues. Results: The administration of taurine resulted in a prolongation of the latency response, its effects being less intense than those of ibuprofen in tail flick test. The use of taurine, was associated by a significant decrease of the behavioral manifestations number, but lesser accentuated than those of ibuprofen in writhing test. Conclusion: Using the mouse model of acute somatic pain, we found that taurine could produce antinociceptive activity, 60 minutes after the thermal noxious tail stimulation. Taurine exhibited, also, a significant analgesic effect in the visceral pain model used in mice.

Keywords: taurine, nociception, tail-flick, writhing test

46. Immunohistochemical Study of Von Willebrand Factor as a Marker of Endothelial Dysfunctions of Utero-Placental Area and Myometrium in Pregnancy

Hoian Vasylivna Anastasiia', Associate Professor Tuleneva Olena', Professor Davydenko Ihor ${ }^{1}$

\section{'Bukovinian State Medical University, Chernivtsi, Ukraine}

Background: The patology of cytotrophoblast invasion, endothelia dysfunctions of utero-placental area (UPA) and myometrium lead to placental ischemia and oxidative stress. Among different methods of examination of UPA and myometrium the most effective is morphological methods, including immunohistochemical staining. Assaying of von Willebrand factor (vWF) permits to study endothelial dysfunctions in different types of blood vessels. VWF is a marker of young endothelial cells, it deposits near the formation of fibrinoid and blood clots (I.Davydenko, 2015). The aim was: to establish the relative immunohistochemical concentration of the vWF in endothelial cells of different types of vessels of the UPA and myometrium in pregnancy. Materials and methods: The biopsy material was obtained by means of standard technique. Multiple paraffin sections ( $5 \mathrm{mkm}$ thickness) were used for three methods: 1)immunohistochemical VWF staining with an antigen thermal exposure; 2)differentiated histochemical fibrin and collagen staining by N.Z.Slinchenko; 3)hematoxylin-eosin staining. Digital images were analyzed with a computer program Image $(1.48 \mathrm{~V}, \mathrm{~W}$. Rasband, National Institute of Health,USA,2015). The evaluation of optical density was based on logarithmic transformations of brightness values. We also calculated the arithmetic mean and its error for optical density (PAST3.06, 0.Hammer, 2015). Results: The most pronounced positive color was observed in formed endothelial cells of the blood vessels, which were stained with varying intensity, in both single blood vessel and different types of blood vessels. The most intensely color was established in arterial type vessels (optical density $0.404 \pm 0.0028$ ), and more in myometrium than in the UPA. Less intense coloration was in the venous type vessels $(0,381 \pm 0,0024)$ and of the microcirculatory system $(0,378 \pm 0,0021)$, however, it did not depend on the localization (myometrium or UPA). The established intensity of coloration of endothelial cells is perspective in studying endothelial dysfunction in vessels of different types, and for arteries - indifferent localization (myometrium or UPA). Moreover, it allows identifying the endothelial cells desquamation as an indicator of huge damage of the intima. Conclusion: Applying an immunohistochemical study of VWF makes possible to assess the degree and nature of endothelial dysfunction of UPA and myometrium vessels for establishing morphological preconditions for fetal insufficiency.

Keywords: uterine-placental area (UPA), myometrium, pregnancy, von Willebrand factor (VWF)

\section{In Vivo Expression and Use of Excitatory Dreadds in a Mouse Epilepsy Model}

Rascu M Miruna', Popovici Patricia Demetria', MariaMiruna Costreie ${ }^{2}$, MD Mihai Stancu33, Associate Professor Ana-Maria Zagrean³, Professor Colin Akerman 4 , MD PhD Andrei Ilie ${ }^{4}$, MD Alexandru Calin ${ }^{4}$, Professor John Jefferys ${ }^{4}$

" Carol Davila" University of Medicine and Pharmacy, Bucharest, Romania, 2University of Glasgow, Glasgow, United Kingdom, "Department of Physiology and Neuroscience,"Carol Davila" University of Medicine and Pharmacy, Bucharest, Romania, 4 Department of Pharmacology, University of Oxford, Oxford, United kingdom

Background: Designer receptors exclusively activated by designer drugs, otherwise known as DREADDs, are a powerful method in neuroscience's toolbox. DREADDs consist of genetically engineered receptors that allow the spatial and temporal control of G-protein coupled signaling through specific synthetic ligands, such as clozapine $\mathrm{N}$-oxide ( $\mathrm{CNO}$ ), which are otherwise inert. Our aim in this study was to express excitatory DREADDs in parvalbumin- and somatostatin-expressing interneurons in the hippocampi of $\mathrm{C}_{57 \mathrm{BL} / 6}$ mice, and to use this experimental system to modulate network excitability in a pharmacological model of epilepsy. Materials and methods: For the procedures we used seven parvalbumin-Cre and six somatostatin-Cre, male and female mice, 
aged 50-200 days. The mice were kept in 12-12 h day/night cycle, with food and water ad libitum. During surgery, two skull openings were made to allow access to both dorsal and ventral hippocampi within each hemisphere. Injections of adenoassociated viruses (AAVs) carrying the double-floxed DREADDs construct were then performed using stereotaxic alignment and a high-resolution glass syringe attached to a micromanipulator. Once both the ventral and dorsal hippocampi were injected with the AAVs, the wound was closed and disinfected. In order to activate the DREADDS, clozapine $\mathrm{N}$-oxide ( $\mathrm{CNO}$ ) was administered prior to the delivery of the epileptogenic agent, 4-aminopyridine. Results: The expression of DREADDs appeared to be robust at 5 weeks after surgery and to continue to beyond 8 months. Furthermore, the activation of interneuron populations via the DREADDs was shown to be capable of modulating seizure-related behaviours in the mouse. Conclusion: Excitatory DREADDs have a long expression window in the mouse hippocampus, potentially allowing agerelated follow-up. Moreover, selective interrogation of specific interneuron types may lead to finding new potential targets for antiepileptic drugs enhancing their efficacy and potency while reducing undesired side effects.

Keywords: chemogenetics, DREADDs, epilepsy, clozapine N-oxide

48. Langerhans Cell Histiocytosis - The Contribution of Anatomopathological Examination

Pasare D. Maria Antoanela', MD PhD Doina Mihaila², Lecturer Elena Cojocaru

"Grigore T. Popa" University of Medicine and Pharmacy, Iași, Romania, "2Saint Mary" County Emergency Pediatric Hospital, Iași, Romania

Background: Langerhans Cell Histiocytosis, also known as Histiocytosis $X$, includes several anatomical and clinical syndromes, which have in common disorganized proliferation, either local or general, of the cells belonging to the reticuloendothelial phagocytic system. Although the aetiology has not been fully understood, viral or bacterial infections are taken into consideration. Due to its characteristic histological, clinical and evolutive features, histiocytosis $X$ is considered a potentially malignant disorder. Case presentation: We present the case of a 7year-old child, without any pathological personal history, that was admitted to the Paediatric Surgery Clinic of Saint Mary Emergency Hospital Iași, for the investigation of a swelling in the frontal area of the head, due to a minor trauma three weeks prior to hospitalization. Radiological examination showed osteolytic lesions of the frontal bone, the left parietal bone, ribs and the right scapulo-humeral joint. An epicranial biopsy from the frontal region was performed. Microscopical examination revealed multiple tissular fragments with a tumoral proliferation consisting of histiocytes, rare granulocytes, scarce multinucleated giant cells, vast areas of necrosis and isolated bone lamellas. Immunohistochemistry showed intensely positive CD1a and CD68 tumor cells. The final diagnosis was Langerhans Cell Histiocytosis. Following informed consent of the family, chemotherapy was initiated in conformity with the protocol. Four weeks after initiation of chemotherapy, the evolution was favourable. Conclusion: Certainty diagnosis for this type of disease is offered only by histopathological examination due to the fact that clinical manifestations are often suited to differential diagnosis with other disorders. Chemotherapy must be taken into consideration in the treatment of these cases.

Keywords: histiocytosis, Langerhans cells, child

49.

Liquid-Based Cervicovaginal Babeș-Papanicolaou CitologyAn Efficient Screening Method for Precancerous Lesions of the Uterine Cervix
Marin M Dragos-Andrei', Onofrei L Andreea1, Miron N Victor Daniel', Claudiu 0 Filimon', Assistant Lecturer Anca Potecă ${ }^{1}$

"Carol Davila" University of Medicine and Pharmacy, Bucharest, Romania

Background: Cervical cancer is caused by human papillomavirus (HPV) and ranks third among all malignancies for women. Highrisk HPV phenotypes, especially 16 and 18, can produce high-grade squamous intraepithelial lesions (HSIL) that can evolve to cervical cancer. Liquid-based cervicovaginal cytology (LBC) represents a screening method for precancerous lesions and cervical cancer. Materials and methods: A retrospective study was conducted on 2454 patients who underwent cervical screening by LBC during January-June 2016. The epithelial cells samples were collected in terms with the protocol of the LBC method and were stained accordingly with the Papanicolaou protocol. These smears were then evaluated using light microscopy and the results were reported using the Bethesda System. Results: The cases were divided into 6 age-groups, the highest percentage of patients $(38.62 \%)$ being included in the $40-50$ age-group. $82.94 \%$ of the cytological examinations were negative for intraepithelial lesions or malignancy (NLIM). The epithelial cells abnormalities had different rates among the age groups. The presence of atypical squamous cells of undetermined significance (ASC-US) was significantly higher in the 30-40 age-group $(p=0.01)$. Low-grade squamous intraepithelial lesions (LSIL) were significantly more frequent in patients younger than 20 years $(p=0.011)$ and in the 20-30 age-group $(p<0.001)$. The patients older than 60 years had significantly more lesions that require further investigations: atypical squamous cells-cannot exclude high-grade squamous intraepithelial lesion (ASC-H) $(p<0.001)$ and high-grade squamous intraepithelial lesions (HSIL) ( $p<0.001)$. The overall incidence of the abnormalities in the analysed cases was as follows: $11.87 \%$ ASCUS, $1.96 \%$ LSIL, $1.18 \%$ ASC-H and $2.05 \%$ HSIL. Conclusion: The precancerous lesions are frequent in Romanian female population, affecting especially young women. The incidence of low-grade lesions is higher in women younger than 30 years, while the highgrade lesions are more frequent in women over 30 years. Early diagnosis of these lesions with high probability of malignant degeneration is the key for decresing the incidence of cervical cancer. $L B C$ is a promising method for cervical cancer screening as it has a higher detection rate for preneoplastic lesions than conventional Papanicolaou smear method.

Keywords: liquid-based cytology, HPV, cervical cancer, screening method

\section{MECOM rs2201862 Polymorphism Increases the Risk of Non} BCR-ABL Myeloproliferative Neoplasms

Voina G Cristian-Marius', MD PhD Adrian P. Trifa'

"Iuliu Hatieganu" University of Medicine and Pharmacy, ClujNapoca, Romania

Background: Polycythemia vera (PV), essential thrombocythemia (ET) and primary myelofibrosis (PMF) are the most frequent BCR-

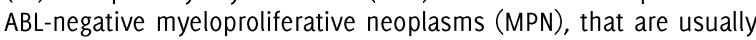
characterized by specific somatic driver mutations, such as JAK2 V617F and CALR. This study aimed to establish the additional contribution of the recently described MECOM rS2201862 polymorphism to the occurrence of MPN. Materials and methods: The study included 939 patients with MPN and 483 individuals ageand gender-matched to the patients, serving as controls. All the experiments have been performed on genomic DNA obtained from peripheral blood withdrawn on EDTA. The genomic DNA was obtained using commercially available kits and the MECOM rs2201862 polymorphism was genotyped in all patients and controls using TaqMan SNP Genotyping assay and run on a QuantStudio 3 real-time PCR machine. Results: The T allele of rS2201862 was associated with the global MPN phenotype (all the three diseases taken together - PV, ET and PMF $)(\mathrm{OR}=1.3 ; 95 \% \mathrm{Cl}=$ 1.1- 1.5; $p$-value $=0.004)$. This effect was observed also when 
analyzing the PV and PMF groups $(\mathrm{OR}=1.3 ; 95 \% \mathrm{Cl}=1.1-1.6 ; \mathrm{p}$ value $=0.004$ and $\mathrm{OR}=1.3 ; 95 \% \mathrm{Cl}=1-1.7 ; p$-value $=0.03$, respectively). A similar trend was noted in the case of ET group, without reaching statistical significance $(O R=1.2 ; 95 \% \mathrm{Cl}=1-1.4 ; \mathrm{p}-$ value $=0.08)$. The $T$ allele of rs2201862 was associated with both JAK2 V617F and CALR-mutated MPN. In the latter case, the effect was considerably stronger $(O R=1.4 ; 95 \% \mathrm{Cl}=1.1-1.8 ; \mathrm{p}$-value $=$ 0.005 versus $\mathrm{OR}=1.2 ; 95 \% \mathrm{Cl}=1-1.4 ; \mathrm{p}$-value $=0.01)$. Conclusion: There was a significant global correlation between rs2201862 and MPN. Regarding the MPN phenotypes, it strongly associated with PV, weaker with PMF, while the association with ET bordered the statistical significance. In ET and PMF, IS2201862 associated especially with CALR-mutated disease. Thus, we may conclude that rS2201862 predisposes especially to PV and to CALR-mutated ET and PMF.

Keywords: myeloproliferative neoplasms, driver mutations, genetic polymorphism, genetic predisposition, MECOM

51. Natural Birth Can Be Taught Using Advanced Medical Simulators

Danciu M.P. Bianca Mihaela', MD PhD Cristian Toma ${ }^{2}$

"'Carol Davila" University of Medicine and Pharmacy, Bucharest, Romania, 2"Prof. Dr. Theodor Burghele" Clinical Hospital, Bucharest, Romania

Background: Nowadays, students usually have less and less access to patients, especially when talking about hands-on maneuvers. Preparing future doctors and improving the trust in executing the basic maneuvers, for example in assisting the natural birth is essential. Medical simulation, by using advanced mannequins, might be a solution in improving their training. Materials and methods: A subjective auto-evaluation was made on 52 foreign students, which have participated at three hands-on workshops held by a simulation center with lots of medical simulation equipment. They were trained Leopold maneuvers, natural rotations of the fetus with plenty of different presentations and full scenarios of natural birth with complications such as: nuchal cord, placenta in different malignant positions. Each maneuver was explained on a different mannequin. Each participant was evaluated by a form before and after the workshop. One by one, the maneuvers were presented by a trained and dedicated doctor in this field. The theoretical part lasted 50 minutes for each maneuver and every participant was able to do it for at least 2 times. Results: Every maneuver was marked with the average between the grade before and after the workshop. We took as well in consideration, the difference between the grading of the self confidence before and after the training. After all, this training was designed for the medical future of the students, so asking some former students about how useful was the workshop for their work directly, managed to highlight one more time the key point: medical simulation is seriously needed! Conclusion: Medical simulation in learning practical skills in foreign students is a very effective method with amazing results. As long as simulation in the medical field is not confused with real medical practice and is only considered a method of improvement, it can bring many benefits.

Keywords: medical simulation training, international students, task-trainer simulators, medical education, natural birth

52. Pharmacologic Influence of the Somatic Nociception Through Nanocarriers Entrapping Dexketoprofen Experimental Data

Damaschin D. Andrei', MD PhD Assoc. Prof. Liliana Mititelu-Tartau ${ }^{2}$

"Grigore T. Popa" University of Medicine and Pharmacy, Iași, Romania, "Department of Pharmacology and Algesiology, "Grigore T. Popa" University of Medicine and Pharmacy, Iași, Romania
Background: Nanotechnology is a field of science, based on the use of materials with nanometric size. The Application of nanoengineering in substance delivery systems design has opened new areas of research in prolonged drugs release. Dexketoprofen is a dextro-enantiomer of the nonsteroidal anti-inflammatory drug ketoprofe and it is a fast-acting analgesic, which is indicated especially by the therapy of painful musculoskeletal diseases, post-surgery conditions, pelvic pain. Data from the literature describe various possibilities for the design of dexketoprofen nanoparticles, which were characterized and studied regarding drug delivery, but only a few were investigated in vivo. Materials and methods: We aimed to evaluate the effects of nanocarriers entrapping dexketoprofen in a somatic pain model in mice. Method: the nanoparticles were obtained by dissolving $L-\alpha-$ phosphatidylcholine in chloroform, removing the solvent by evaporation, and hydrated thereafter by adding distilled water with dexketoprofen. The soft matter vesicles were stabilized with a $0.5 \%$ chitosan solution, which acts like a supplementary transport barrier, allowing the slow release of the substance entrapped inside. We evaluated the distribution of vesical dimensions and the Zeta potential using a Malvern Zetasizer Nano ZS ZEN-3500 apparatus. To direct viewing of the nanoparticles, a Nikon Ti Eclipse optical microscope was used. The experiment was carried out on white Swiss mice (20-25g), divided into 3 groups of 7 animals each, treated orally: Group I (Control)- distilled water $0.1 \mathrm{ml} / 10 \mathrm{~g}$ body weight (bw), Group II (DEX)- dexketoprofen $0.15 \mathrm{mg} / 10 \mathrm{~g}$ bw, Group III (DEX-ves)- dexketoprofen vesicles $10 \mathrm{mg} / 10 \mathrm{~g}$ bw. Antinociception was assessed using the hot-plate test, in order to evaluate the latency time reactivity to thermal noxious stimulation. Experimental protocol was implemented according to recommendations of our University Committee for Research. Results: The obtained nanoparticles were found to have a mean size of $368 \mathrm{~nm}$ and mean Zeta potential of $+61,7 \mathrm{mV}$, thus suggesting a very good stability of the solution. The administration of dexketoprofen vesicles was associated with an increase of the latency response, statistically significant in the interval between 2 and 8 hours in the experiment. Conclusion: The use of nanovesicles as carriers for dexketoprofen resulted in a sustained release of the drug, compared to a non-entrapped agent in the hot-plate test on mice.

Keywords: dexketoprofen, nanoparticles, hot-plate, mice

\section{Psychosomatic Features of Complicated Grief Disorder (CGD)}

Tsagkaris G. Christos', MD PhD A. Kamaratos ${ }^{2}$

${ }^{7}$ Faculty of Medicine, University of Crete, Crete, Greece, ${ }^{2} 75 t$ Internal Medicine Department, Tzaneio Prefecture General Hospital, Pireas, Greece

Background: CGD has recently been established in DSM 5 illustrating the need of a biomedical classification of grief associated functional disorders. The behavioral features of CCD have been widely discussed; however, there is little evidence on body functions alterations associated with CGD. Case presentation: K.N. is an 80 y.o. man who experienced the loss of his grandson one year ago. He has history of diabetes mellitus type II and coronary heart disease. K.N. presented loss of walking ability few days after the incident which was associated with the shock stage of Grief and Loss classification. Ceneral evaluation as well as neurological and orthopedic assessment showed no pathological causes of this impairment indicating its psychological background of. Physical therapy sessions did not make an impact as well. As part of the patient's psychological assessment Complicated Grief Disorder Questionnaire proved positive (CGDQ Score >or $=5$ ). In the meantime, the patient refused to follow a proper diet decreasing his food in several weeks intervals. An E. Coli urinary infection was established in June 2017 and urinary cultures were positive until October 2017 even if antibiotics have been administered properly. The patient referred no previous resistant infection and suffered from several complications of this specific infection. After being admitted in the hospital the infection was treated; An 
ongoing decrease in cortisol levels was noticed in his laboratory examinations. K.N. seems willing to walk but he is unable to do so for now. Conclusion: K.N.'s case presents special interest given that motility impairment is a rare complication of CGD which may be interpreted through the newly established field of psychoneuroendocrinology. Cortisol's decline could justify the general inactivity leading to loss of walking and the vulnerability of his towards urinary infection. K. N's unwillingness to be nourished in a proper way appears as a dysbiosis factor inhibiting the immunosuppression of $\mathrm{E}$. Coli which could immigrate and multiply. It is also likely that grieving switched negatively lymphocytes' activity. All in all the biology of behavior ought to be further investigated focusing on both noticeable cases and prospective studies

Keywords: complicated grief disorder, psychosomatic disorders, psychoneuroendocrinology

54.

Teaching Anesthesia Procedures with Medical Simulators for International Students in Romania

Eftenoiu D Anca-Elena', MD PhD Cristian Toma ${ }^{2}$

"Carol Davila" University of Medicine and Pharmacy, Bucharest, Romania, 2"Prof. Dr. Theodor Burghele" Clinical Hospital, Bucharest, Romania

Background: Medical students encounter more and more difficulties when trying to practice on patients. In addition, international students have to overcome the language barrier too. Improving the knowledge, skills and self-confidence when performing clinical anesthesia techniques must be a goal of medical education starting from the university years. Medical simulation can be a useful approach to improving their training in the practice of anesthesia. Materials and methods: In this study we performed a subjective self-evaluation of 64 foreign students who participated in 4 hands-on workshops in a medical training facility. They performed the following techniques: placing an oropharyngeal airway, bag-valve-mask ventilation, endotracheal intubation, handling a laryngoscope, lumbar puncture and central venous catheterization on task-trainer simulators. Each participant has been evaluated before and after the workshop through a standard form. Techniques had been firstly presented by a trained person and each student performed the procedure at least twice. Results: All participants showed improvement in procedural skill and self-confidence. They were more likely to offer to perform these techniques on patients during the hospital training program. They had better understanding of the practice of anesthesia. Conclusion: Simulation-based training is an effective method of teaching anesthesia advanced clinical skills to international medical students. Due to the wide array of practical skills that should be learned by a resident physician during residency, such abilities should be implemented starting from the university years.

Keywords: medical simulation training, international students, task-trainer simulators, medical education, anesthesia

The Biceps Brachii Muscle and Its Distal Insertion: Footprint, Measurements and Clinical Relevance

Sugeac S Anca', Dangulea V. M. Teodora Andreea', TartaArsene Eugen ${ }^{1}$

"Carol Davila" University of Medicine and Pharmacy, Bucharest, Romania

Background: Anatomists have considered until recently the insertion of the brachial biceps to be unique. It originates on the scapula through two ends that join together to form a muscular body, inserting on the radial tuberosity through a common tendon. The purpose of this study is to map the footprint of the brachial biceps tendon insertion on the bicipital tuberosity and to demonstrate the separate insertion of the tendons of the two ends (long and short) of the muscle in order to assist surgeons with correct tendon orientation during surgery. Materials and methods:
Five upper extremities from three cadaver specimens in the Department of Anatomy of the "Carol Davila" University of Medicine and Pharmacy, Bucharest were used and we followed through dissection techniques the route of the tendons of the two heads and the way they insert on the radial tuberosity. We studied them in pronation, semiflexion and flexion. Also, in order to confirm the information we analyzed a series of RM investigations of the brachial region and also the elbow region. The length, width and area of the biceps tendon insertion were measured. Results: The brachial biceps originates on the scapula: the short head has its origin on the coracoid process through a common tendon with the coracobrachial muscle, and the long head originates on the supraglenoid tuberus of the scapula. We observe the insertion of the long head tendon into the proximal region of the radial tuberosity, while the short head tendon is inserted into the distal region of the tuberosity, thus contradicting the idea of a single insertion. We also highlight the way of solidarization of the two ends by means of a fascia, as well as fibers passing from the long head to the short head. Conclusion: The evaluation of the brachial biceps footprint of insertion will allow anatomic orientation and improve the anatomical approach in the distal tendon repair surgical interventions. The distal short head of the biceps has a consistent relationship with the bicipital aponevrosis and distinct distal insertion from that of the long head on the bicipital tuberosity.

Keywords: brachial biceps, short head, long head, bicipital aponevrosis, radial tuberosity, distal insertion, muscular body, supraglenoid tuberus, coracoid process

\section{The Effects of Ascorbic Acid Long-Term Administration on Pain Models in Mice}

Schreiner R.C. Thomas-Gabriel', Schreiner R Oliver Daniel ${ }^{1}$, Catan F Lilia', Strat S Silvia Cristina', Assistant Lecturer Andrei Luca

"Grigore T. Popa" University of Medicine and Pharmacy, lași, Romania

Background: Ascorbic acid is an essential micronutrient that serves as a cofactor in a number of enzymatic and chemical pathways. It acts as an antioxidant by inhibiting the formation of reactive oxygen species (ROS) which are reported to be associated with chronic pain, especially neuropathic and inflammatory pain. The aim of our study was to assess the effects of long-term administration of Ascorbic acid on formalin-induced pain and on acetic acid-induced visceral pain. Materials and methods: 14 $\mathrm{BALB} / \mathrm{C}$ mice were divided into two groups. The mice in the first group received $500 \mathrm{mg} / \mathrm{kg}$ b.w. of Ascorbic acid via intraperitoneal administration for 21 days. The second group served as control group and received an equal volume of distilled water in the same manner. To evaluate the analgesic effect, acetic acid Writhing Test (Visceral Pain Test), Paw Formalin Test and Orofacial Formalin Test were performed 24 hours after the last substance administration. The results were compared using the Student's t-test. Results: Ascorbic acid has shown analgesic effects on the Visceral Pain Test, as compared to the control group $(p=0.05)$. On Paw Formalin Test, ascorbic acid has shown an analgesic effect on both phases of the test (phase 1 and phase $2 \mathrm{p}=0.01$ ). However, on Orofacial Formalin Test, it demonstrated analgesic effects only on the second phase $(p=0.01)$ compared to the control group. Conclusion In our study we demonstrated that long-term administration of Ascorbic acid modulates pain perception in visceral and formalininduced pain. However, further studies are required to assess the exact mechanisms by which this antinociceptive effect occurs.

Keywords: ascorbic acid, nociception, visceral pain, formalininduced pain 
57. The Evaluation of Omeprazole-Isoniazid Interaction on Experimental Model of Indomethacin-Induced Ulcer in Mice

Ilășoaia F Amalia - Elena', Zaharia I Ioan', Axinia V IuliaGeorgiana', MD PhD Magda Costin', Associate Professor Veronica Bild'

"Grigore T. Popa" University of Medicine and Pharmacy, Iași, Romania

Background: The working hypothesis was based on the fact that in medical practice patients diagnosed with gastric ulcer or gastritis and hyperacidity frequently receive administration of omeprazole. The duration of the combination simultaneously with isoniazid and omeprazole varies from the type of digestive pathology, duration of the treatment and with other associated pathologies. This paper attempts to assess the interaction between background therapy with isoniazid and use of omeprazole in gastric ulcer induced by chemical agents on mice. Materials and methods: Swiss albino mice were used and kept in special cages at controlled temperatures, with a light/dark cycle of 12/12 hours and with ad libitum access to food and water. Food supply was stopped 24 hours before ulcer induction. The mice were grouped accordingly: Lot I - Mucilage of tragacanth $2 \%$ p.o.(per os), Lot II - single dose of indomethacin $18 \mathrm{mg} / \mathrm{kg}$ p.o. , Lot III - single dose of indomethacin $18 \mathrm{mg} / \mathrm{kg}+$ omeprazole $3 \mathrm{mg} / \mathrm{kg} \mathrm{p.0,} \mathrm{Lot} \mathrm{IV} \mathrm{-} \mathrm{single}$ dose of indomethacin $18 \mathrm{mg} / \mathrm{kg} \mathrm{p.0.} \mathrm{+} \mathrm{isoniazid} 15 \mathrm{mg} / \mathrm{kg} \mathrm{p.0.,} \mathrm{Lot}$ $\mathrm{V}$ - single dose of indomethacin $18 \mathrm{mg} / \mathrm{kg}$ p.o + omeprazole $3 \mathrm{mg} / \mathrm{kg} \mathrm{p.0}+$ isoniazid $15 \mathrm{mg} / \mathrm{kg}$ p.o. concomitant, Lot VI - single dose of indomethacin $18 \mathrm{mg} / \mathrm{kg}$ p.o. + omeprazole $3 \mathrm{mg} / \mathrm{kg} \mathrm{p.o.} \mathrm{+}$ isoniazid $15 \mathrm{mg} / \mathrm{kg}$ p.o., with one hour between administrations. The experiment was conducted over a period of 7 days to sacrifice 5 animals from each group, harvesting the organs of interest and preparing them for macroscopic and microscopic examination. After each step, the ulcer index was calculated. The experimental protocol was conducted in compliance with internal rules approved by "Grigore T.Popa" University of Medicine and Pharmacy laşi and international bioethical norms providing working with laboratory animals (Directive 63/2010). Results: By comparing lot III with the other study groups using ANOVA test, Newman-Keuls test there were obtained statistically significant data $(p<0.05)$ for lot $V$ and very statistically significant data $(p<0$, 01) for lot VI. Conclusion: The data obtained by comparing the lot III with lots V and VI confirms the need to establish in parallel a proper treatment for ulcer disease under treatment with isoniazid.

Keywords: gastric ulcer, indomethacin, omeprazole, isoniazid

58. Upper Gastrointestinal Endoscopy Simulation Techniques for International Medical Students in Romania

Ciocan A Alida Teodora', MD PhD Cristian Toma ${ }^{2}$

I"Carol Davila" University of Medicine and Pharmacy, Bucharest, Romania, 2"Prof. Dr. Theodor Burghele" Clinical Hospital, Bucharest, Romania

Background: Medical students have access to patients, but they show difficulties when trying to practice on them. International students have to overcome the language barrier too. Practical skills and self-confidence when performing clinical endoscopy procedures must be aquired starting from the university years. Medical simulation teaching can be a useful way to improving their training in the practice of endoscopy. Materials and methods: In this study we performed a subjective self-evaluation of 24 foreign students who participated in 2 hands-on workshops in a medical training facility. They performed the following techniques: three games for developing fundamental endoscopic skills and an upper gastrointestinal endoscopy on a high-fidelity endoscopic simulator. Standard questionnaires were applied for each participant before and after the workshop. Firstly, a trained person presented every technique and then each student performed the procedure at least twice. Results: Procedural skills and selfconfidence have improved in every participant. They were more likely to want to perform these endoscopic techniques on patients during the hospital training program. They had better understanding of the practice of upper gastrointestinal endoscopy. Conclusion: Simulation-based training is a good method of teaching endoscopy advanced clinical skills to international medical students. Due to the need of practicing these endoscopic maneuvers by a resident physician during residency, such abilities should be implemented starting from the university years.

Keywords: medical simulation training, international students, task-trainer simulators, medical education, high-fidelity endoscopic simulator

\section{Uterine Leiomyosarcoma - A Rare Malignancy}

Maria C Enea', Cretu S Manuela', Dascalescu F Andra', MD PhD Prof. Raluca Balan', MD PhD Cornelia Amalinei' "Grigore T. Popa" University of Medicine and Pharmacy, lassi, Romania

Background: Uterine leiomyosarcoma (LMS) is a rare uterine tumor that arises from the uterine smooth muscles. It accounts for $1-2 \%$ of all uterine malignancies. Although this tumor has morphological and clinical features similar to other uterine benign and malignant entities, the accurate diagnosis is necessary, due to their different behavior, management, and prognosis. LMSs are considered unpredictable, with a relative resistance to oncologic treatment. Case presentation: We describe the case of a 48 year-old woman admitted for 3 months metrorrhagia. Anamnesis showed no family history of cancer and no related risk factors. Abdominal exam revealed a tender and painless $10 \mathrm{~cm}$ diameter polycyclic mass located in the hypogastric region. The uterus was enlarged, with polycyclic outline, in digital vaginal exam, feature which was confirmed by ultrasound exam. The laboratory data have been that of a microcytic hypochromic anemia and an inflammatory syndrome. The clinical diagnosis was that of hemorrhagic uterine fibroid, secondary anemia, and fibrocystic breast disease. The treatment consisted of total abdominal hysterectomy with bilateral salpingo-oophorectomy. Postoperative evolution was uneventful. Grossly, the tumor presented as a poorly defined submucosal nodule, with a soft consistence, measuring $6 \mathrm{~cm}$ in diameter. The microscopic examination of this tumor nodule revealed fascicles of spindle cells, with pleomorphic hyperchromatic nuclei exhibiting moderate to severe atypia, areas of tumor necrosis, and at least 5 atypical mitoses/ $10 \mathrm{HPF}$. Immunohistochemistry technique for ER, PR, PCNA, SMA, P53, and bcl-2 has been performed for the differential diagnosis. The final histopathological diagnosis was that of uterine LMS. The particularity of our case was the association with ovarian endometriosis and serous cystadenoma. From our knowledge, there are no literature reports of this pathological association, possible due the reduced prevalence of this type of uterine malignant tumor. Conclusion: Uterine LMS is a rare tumor with an unpredictable, aggressive behavior, showing different histological subtypes, thus thorough follow-up and careful histopathological differential diagnosis is necessary. $E R, P R$, $\alpha-S M A, P C N A, \quad$ 553, and $b c l-2$ represent a reliable panel of antibodies for both positive and differential diagnosis of LMS. The association with endometriosis and cystadenoma suggests possible common pathogenic pathways and envisages future targeted therapies.

Keywords: Uterine Leiomyosarcoma, immunohistochemistry, smooth muscle

60. Acute Mesenteric Infarction- A Short Insight into the Pathophysiology and Current Diagnosis

Gălămîj 0-M Iulia-Maria', Chiriță - George-Alexander ${ }^{1}$, Lecturer Lupu George ${ }^{1}$, Assistant Lecturer Bratu Matei ${ }^{1}$

"Carol Davila", University of Medicine and Pharmacy, Bucharest, Romania

Background: Acute mesenteric infarction (AMI), with its four etiologies, (mesenteric arterial occlusions, which are embolus and 
thrombosis, mesenteric venous occlusion, and nonocclusive processes, especially vasospasm) presents problems regarding the early diagnosis and a prompt, efficient treatment. The aim of our study is to establish a description of current diagnostic possibilities and increase awareness among future doctors. Materials and methods: This is a review of the literature concerning the pathophysiology and diagnosis of AMI. We did an Online medical databases research with the relevant keywords and we selected only high impact studies. Clinical signs and imagistic diagnosis data were recorded and summarized and presented as results. Acknowledged risk factors for acute mesenteric ischemia include cardiac arrhythmias, advanced age, low cardiac output states, generalized atherosclerosis, congestive heart failure, severe valvular cardiac disease, recent myocardial infarction, and intraabdominal malignancy. Clinical scenario differentiates AMI as mesenteric arterial emboli, mesenteric arterial thrombosis, nonocclusive or mesenteric venous thrombosis. Results: The most relevant, recent and high-impact studies comprised the pathophysiology of the four etiogies, common and specific clinical manifestation and best imagistic aproaches for an accurate and early diagnosis. Whatever the cause, the results of intestinal ischemia are the same: a spectrum of injury ranging from completely reversible functional alterations to transmural hemorrhagic necrosis of portions or all of the bowel. Diagnosis is suspected when sever, sudden pain in the middle part of the abdomen or back, guaiac positive stools, leukocytosis and scarcity of gas in the bowel on x-ray study appear in a patient with recent myocardial infarction, or previous arterial emboli. Diagnostic investigations that are strongly suggestive are: selective arteriography preceded by an aortogram, determination of serum alkaline phosphatase, lactate or phosphate levels, abdominal radiography or ultrasounds (US), Doppler US, fluoroscopic singlecontrast examinations and last but not least, computed tomography (CT) without oral administration of contrast material, which has replaced conventional angiography as the "gold standard" imaging modality in AMI. Conclusion: Despite improvements in clinical recognition, intestinal infarction remains a devastating event. However, there is a potential for improved outcome, even in this life-threatening vascular catastrophy.

Keywords: acute mesenteric infarction, embolus, thrombosis, cardiac arrhythmias, atherosclerosis, ischemia, myocardial infarction, computed thomography

61. Amyotrophic Lateral Sclerosis - Initial Symptoms and Evolution

Efremov G Mihaela', Spoială L Maria', Associate Professor Sangheli Marina'

'Nicolae Testemiţanu State University of Medicine and Pharmacy, Chişinău, Republic of Moldova

Background: Amyotrophic lateral sclerosis (ALS) is an idiopathic, fatal, neurodegenerative disease that results in progressive loss of bulbar and limb function. Approximately $75 \%$ of the patients with typical ALS have a spinal form of the disease (limb onset) and present with symptoms related to focal muscle weakness and wasting, where the symptoms may start either distally or proximally in the upper and lower limbs. Patients with bulbar onset ALS usually present with dysarthria and dysphagia for solid or liquids, and limbs symptoms can develop almost simultaneously with bulbar symptoms, and in the vast majority of cases will occur within 1-2 years. Paralysis is progressive and leads to death due to respiratory failure within 2-3 years for bulbar onset cases and 3-5 years for limb onset. Materials and methods: It was conducted a descriptive retrospective study over a 8 year period (2009 to 2016) on 44 pacients with diagnosis ALS, hospitalized in the Institute of Neurology and Neurosurgery from Chişinău. Results: The study included 25 men (56,82\%) and 19 women $(43,18 \%)$ with a mean age of 52.9 , (range 28-68). The average of undiagnosed period is 14,38 months (range $2-72$ ). The results showed that 32 patients $(72,72 \%)$ had spinal onset, and 12
$(27,27 \%)$ had bulbar onset. The most common onset symptoms are: muscle weakness- $34(77,27 \%)$, fasciculations- $11(25 \%)$ muscle atrophy- $6(13,63 \%)$, dysphagia- 11 (25\%), dysarthria- 14 $(31,81 \%)$, dysphonia- $9(20,45 \%)$. The disease is progressive so at the moment of diagnosis we had the following results: weakness$41(93,18 \%)$, fasciculations- $32(93,72 \%)$, muscle atrophy- 26 $(59,09 \%)$, dysphagia- $33(75 \%)$, dysarthria- $30(68,18 \%)$, dysphonia$32(72,72 \%)$, and shortness of breath- $6(13,63 \%)$. Conclusion: Amyotrophic lateral sclerosis is a syndrome of progressive deterioration involving the corticospinal tract, brainstem, and anterior horn cells of the spinal cord. The rapidly progressive course of ALS means that the patient's level of disability changes over a timescale of months rather years. An urgent need remains for the identification of early biomarkers of disease onset and progression, and efficient approaches to early-phase clinical trials are required to accelerate the identification and development of useful therapies for ALS.

Keywords: ALS, bulbar onset, spinal onset, onset symptoms

\section{An Uncommon Case of Duodenal Stenosis in Patient with} Chronic Heroin Abuse and Chronic Hepatitis C Borcan G.C. Andrei-Mihai', Ayatollahi H.R. Simin², Iancu L.M. Oana ${ }^{2}$, Associate Professor Ciornei Mariana Catalina 'Carol Davila' University of Medicine and Pharmacy, Bucharest, Romania, ${ }^{2 T i t u}$ Maiorescu University, Bucharest, Romania

Background: Hepatitis C incidence is high in young injecting drug users; HCV is associated with lymphoproliferative disorders such as mixed cryoglobulinemia and non-Hodgkin's lymphomas; the prevalence of Hepatitis C Virus infection in non-Hodgkin's lymphoma is 7,4-37\%; Non-Hodgkin's lymphoma is frequently found at the gastric level; HCV infection is associated with an increased risk of pancreatic cancer. Case presentation: 38-year-old male patient with known twenty years long drug abuse and chronic hepatitis C infection presents to the Emergency Room for nausea, incoercible vomiting and severe weight loss. Repeated endoscopic examination revealed stenosis of the second part of the duodenum. Through endoscopic means, tissue fragments were also obtained, and through the first histopathological examination, adenocarcinoma was revealed. The CT exam showed a normal pancreas with no abdominal lymphadenopathy, circumferential swelling of the second duodenum, a very distended stomach and normal aspect of the gallbladder, liver and spleen. When admitted, the patient was severely cachexic, and kidney failure with severe hyponatremia, hypokalemia and hypochloremia were discovered. The patient underwent gastroenteroanastomotic surgery, so that nutrition would be possible, improving the biological status in order to perform a second surgery, for the removal of the tumoral obstruction. A second histopathological exam was performed, confirming a highly differentiated adenocarcinoma. Conclusion: Chronic IV drug abuse, in a low social status patient increases the risk of hepatitis $C$ virus infection and thus compromises the immune system, making it less effective against tumor development.

Keywords: duodenal stenosis, drug abuse, hepatitis C

\section{An Unusual Case of Transient Aggravation of Dyspnea}

Cojocaru C.C Andra-Maria', S.D. Teona-Maria', Lecturer Mariana Floria

I"Gr. T. Popa" University of Medicine and Pharmacy, Iassi, Romania

Background: The pacient was hospitalized for a transient aggravation of chronic dyspnea. Due to his personal pathological history we suspected pulmonary thromboembolism, diagnosis disproved by medical imagistic. Case presentation: A 76-year-old man with chest pain and transient aggravation of chronic dyspnea was hospitalized through emergency room. He had medical history of chronic cor pulmonale due to pulmonary fibrosis. Electrocardiogram showed atrial fibrillation with fast ventricular 
rate, right hyper axis deviation and is in all precordial leads; in one previous electrocardiogram the patient has had left bundle branch block. He was under chronic oral anticoagulation (acenocumarol), but not in therapeutic range. Due to the high suspicion of pulmonary thromboembolism a CT-scan chest was recommended. No thrombus in pulmonary artery circulation was detected. However, the cross section of the coronary sinus showed a dilatation and an irregular mass of about $8,3 \mathrm{~mm}$, which was most likely a thrombus. Transthoracic echocardiography revealed dilated right ventricular cavities and pulmonary artery systolic pressure of 75-80 mmHg. Parasternal long views confirmed dilated coronary sinus with a visible thrombus in a dilated coronary sinus, without spontaneous contrast in the right or left cardiac cavities. No coronary sinus lead or other invasive cardiac procedures experienced our patient. After a few days of intravenous heparin, a novel oral anticoagulant was initiated. After 24 hours the patient improved and after 7 days coronary sinus thrombus disappeared on the transthoracic echocardiography. Conclusion: In patients with dilated coronary sinus, thromboembolic risk and inappropriate anticoagulation, a more attentive examination on transthoracic echocardiography is mandatory in case of acute dyspnea, even in the absence of spontaneous contrast.

Keywords: chronic dyspnea, anticoagulation, thromboembolism, transthoracic echocardiography

64. Biological Therapy - An Effective Treatment in Severe Psoriasis Associated with Virus C Hepatic Infection?

Branisteanu D Catalina', Bianca-Gabriela C.E Bostan', Branisteanu Daciana Elena'

'Grigore T. Popa University of Medicine and Pharmacy, Iassi, Romania

Background: In the treatment of psorlaşis, a number of biological molecules are used with favorable results, some approved for years, others newer, of which we mention the anti-TNF alpha agents and IL-12/IL-23/IL-17 antagonists. The mechanism of action of these agents consists in blocking some specific steps in the cascade of molecular events involved in the pathogenesis of this complex disease.

Case presentation: We present the clinical case of a 69-year-old female patient known since 1978 with rheumatoid arthritis and who developed severe psorlaşis vulgaris in 1995. Over time, the patient was on multiple therapeutic schemes for rheumatoid arthritis (gold salts, methotrexate, sulfasalazine) without improvement of the joint lesions. In May 2008, biologic therapy with etanercept was initiated and then discontinued 5 month later due to the development of a left pleural empyema with multiple adhesions. A month after the last administration of etanercept, the patient developed a pathologically confirmed as nodular hypodermitis. Since 2010, due to the unfavorable response of skin and joint lesions the patient was switched to adalimumab, with a slow, but favorable outcome. Regarding the joint disease, in January 2013 the rheumatologist decided to associate methotrexate, $7.5 \mathrm{mg} /$ week, which resulted in the stoppage of further development of degenerative joint changes and persistence of mild arthralgia. Based on the specific serologic assays a diagnosis of hepatitis C virus infection was made. Since the initiation of the first biologic therapy till now the patient did not present HCV infection-related alterations of liver function. Conclusion: HCV infections make doctors reluctant to recommend biologic therapy. However, based on the analysis of the disease course on adalimumab in our patient we believe that HCV infection should not constitute an impediment to the administration of biologic therapy for severe psorlaşis, especially when associated with joint damage. The association of rheumatoid arthritis with severe psorlaşis vulgaris and HCV infection worsens the prognosis of diseases and the therapeutic success. Despite this, during the almost 7 years of adalimumab therapy, the efficacy and safety profile of this treatment have proved to be very good, with good control of skin and joint lesions and absence of hepatocytological changes.

Keywords: psoriasis, biological therapy, hepatitis C virus infection, rheumatoid arthritis

\section{Challenges in the Diagnosis of Heart Tumors-Insights from} a Case

Strat S Silvia Cristina', Maga V. Cristina', Schreiner R.C. Thomas-Gabriel', Schreiner R Oliver Daniel', Professor Ciprian Rezus², Assistant Lecturer Codruta Badescu², MD PhD Elena Mitrea

"Crigore T Popa" University of Medicine and Pharmacy, lassi, Romania, ${ }^{2}$ St Spiridon Emergency Hospital, IIIrd Clinic of Internal Medicine

Background: Primary cardiac tumors are uncommon, but not extremely rare. The variety of cardiac tumors ranges from nonneoplastic lesions to high-grade malignancies which occur over a wide range of ages. Based upon the data of 22 large autopsy series, the prevalence of primary cardiac tumors is approximately $0.02 \%$. About $75 \%$ of primary tumors are benign, and $50 \%$ of benign tumors are myxomas. Approximately $20 \%$ of patients are asymptomatic, with tumors being found incidentally on echocardiography. Sudden death may occur in $15 \%$ patients with atrial myxoma, typically caused by coronary or systemic embolization or by obstruction of the mitral or tricuspid valve. Case presentation: We present the case of a 57 year-old Caucasian female with an unremarkable personal medical history. Family medical history includes sudden death of the father at age 49 (cardiovascular cause- no details available). The patient is admitted with progressive dyspnoea, a chronic productive cough, progressive fatigue, occipital cephalalgia and vertigo. Preliminary examination, EKG, chest radiograph and laboratory blood investigations set the following diagnosis: stage III essential hypertension, stage III chronic obstructive pulmonary disease (COPD), pulmonary hypertension, morbid obesity, hypercholesterolemia and hepatic steatosis. Routine cardiac ultrasonography shows a hyperechogenic, $41.72 / 44.5 \mathrm{~mm}$ left atrial mass, attached through a pedicle to the interatrial septum, causing partial obstruction of the mitral valve. The patient was referred to the 'Prof. Dr. George I.M. Georgescu' Cardiovascular Diseases Institute for surgical treatment. Histopathology revealed left atrial myxoma, setting a good prognosis for the condition. Conclusion: Although most cardiac tumors are benign, because of their malignant potential, the risks secondary to impaired cardiac function, conduction system involvement, and/or peripheral embolism mandate prompt evaluation and definitive treatment. Primary cardiac tumors remain an unexpected challenge for the clinicians and surgeons because of their rarity and the lack of clinical guidelines. The present report illustrates the poor symptomatology of a large left atrial myxoma. Death is attributed to embolization or mechanic obstruction of the mitral valve. Prompt surgical treatment sets a good prognosis and annual echocardiography is mandatory due to recurrence risk.

Keywords: myxoma, asymptomatic, ultrasonography, heart tumor

\section{Chronic Inflammatory Demyelinating Polyneuropathy as an} Initial Manifestation of Crohn's Disease.

Turac I János-Robert'́, G Cristina Maria', Lecturer Dr. Lidia Ciobanu'

"Iuliu-Hatieganu" Univeristy of Medicine and Pharmacy, ClujNapoca, Romania

Background: Crohn's disease (CD) is a relapsing transmural inflammatory disorder that can affect the entire gastrointestinal tract from the mouth to the anus. It is considered to be a systemic disease, as it has many extra-intestinal manifestations. Peripheral neuropathy (PN) is one of the most frequently reported neurological complications, however, the clinical and pathological 
features of Chronic Inflammatory Demyelinating Polyneuropathy (CIDP) associated with $C D$ remain incompletely understood. Case presentation: A 22-year-old female, was admitted 3 years ago with ascending weakness of both lower extremities that had progressed for about two weeks. On neurologic examination, she had symmetric weakness of both lower extremities with paresthesia of both hands and feet. After several investigations, she was diagnosed with CIDP, being under cortisone and immunosuppressive treatment. In April, she presented with a superior digestive hemorrhage externalized as hematochezia. In the Intensive Care Unit, endoscopic investigations were performed, without any concluding sources of hemorrhage, but some ulcerative lesions of about $2 \mathrm{~mm}$ in the terminal ileum were observed for which the suspicion of $C D$ was raised. The suspicion was confirmed by an entero-CT and biopsy. Currently she is under treatment with Azathioprinum, Methylprednisolonum, and she was advised to undergo surgery with a chance to treat both $C D$ and CIDP. Conclusion: We report a case in which CIDP was the initial presentation of concealed CD. CIDP is an autoimmune disease, therefore we should consider inflammatory bowel disease as a hidden possible concurrent or preceding disease when a patient has recurrent episodes of CIDP. Taking into consideration that only a small part of the terminal ileum was affected, surgical resection of this segment was recommended. Considering the CIDP an extraintestinal manifestation of CD, remission of CIDP is expected after the surgical procedure and appropriate long-term treatment for CD.

Keywords: Crohn's Disease, Chronic Inflammatory Demyelinating Polyneuropathy, Inflammatory Bowel Disease, Extra-intestinal manifestations, Surgical resection

\section{Diagnose in Stargardt's Disease}

Preoteasa D Laura Denisa', Dumitru G Alida Nicoleta', Claudia A Mihail', MD PhD Preoteasa Dana

"Carol Davila" University of Medicine and Pharmacy, Bucharest, Romania

Background: Stargardt's Disease is a rare inherited retinal disorder transmitted as an autosomal recessive trait. It affects the central region of the retina called "macula", which is specialized in highacuity vision. In this condition, toxic forms of Vitamin $A$ are produced, which build up in lipofuscin granules in the retinal pigmented epithelium. Symptoms, which typically appear in late childhood, include progressive vision loss and impaired light adaptation. Materials and methods: The following study presents the clinical characteristics of a family who developed Stargardt's Disease in both eyes. The family consists of 2 brothers and 2 cousins, who started losing their vision from 10-12 years old. All patients received a full ophthalmological examination including visual acuity tests, ocular fundus, visual field and macular OCT (ocular computed tomography) in correlation with genetic tests. These investigations are judging the extent of macular atrophy and risk of blindness which are common signs in the 4 members. The final diagnosis is based on chromosomal mutations. Results: The acuity visual test shows less than $20 \%$ ocular capacity and visual field presents an absolute central scotoma. The macular OCT reveals central atrophy with complete loss of the photoreceptors' layer in the foveal region. Central foveal thickness was reduced in 3 patients below $50 \%$. One patient was diagnosed with subretinal fibrosis and intraretinal liquid. Genetic tests revealed a mutation of the $A B C A 4$ gene, which causes the formation of toxic vitamin $A$ dimers. Conclusion: We remarked that all signs of Stargardt's Disease are encountered: loss of vision from early age, macular atrophy and especially the mutation of $A B C A 4$ gene, thus being able to establish a certain diagnose. Unfortunately, no remedy is known and additional research is needed in order to improve the visual prognosis.

Keywords: macular atrophy, Stargardt's Disease, photoreceptors, genetic mutation, macular OCT

\section{Endobronchial Tuberculosis in a Teenager with Unilateral} Wheezing and Persistent Cough

Tabacu M Iulia-Andreea', Bărboi I Teodora-Maria', Lungu M Laura Maria', MD PhD Emilia Tabacu',

"Carol Davila" University of Medicine and Pharmacy, Bucharest, Romania, 2"Marius Nasta" Institute of Pneumology, Bucharest, Romania

Background: Tuberculosis alongside human immunodeficiency virus (HIV) is considered a leading mortality cause worldwide. Endobronchial tuberculosis (EBTB), also known as tracheobronchial tuberculosis is a special form of tuberculosis, being defined as the infection of the tracheobronchial tree with Mycobacterium tuberculosis. The diagnosis is difficult to establish, since the chest radiograph is frequently inconclusive and as an unfortunate result the treatment might be delayed. The best diagnostic tool is bronchoscopy, which can show bronchial lesions such as stenosis or obstructions. Systemic symptoms such as appetite and weight loss and generalised weakness are accompanied by cough, fever (usually low grade) and sometimes localized wheezing. However these signs do not point directly toward the EBTB diagnosis, since they simulate other diseases such as bronchial asthma, cancer, and recurrent pneumonia. Case presentation: A 19 year-old female, non-smoker, with no past medical history presents to the pulmonologist with a 4 month persistent cough, dysphonia and wheezing that is non-responsive to antibiotics, bronchodilators, and corticosteroids. Her father died of pulmonary cancer. The chest radiograph was normal, so the following step was the bronchoscopy procedure that showed irregular mucosa imflammation, caseating and granular lesions of the trachea and the right bronchial tree, bronchial stenosis of the left main bronchus and enlarged carina. The bronchial biopsy as well as the bronchial aspirate underwent Ziehl-Neelsen staining and revealed the acid-fast bacilli of Mycobacterium tuberculosis. She started a four-drug treatment consisting of isoniazid, rifampicin, ethambutol and pyrazinamide, along with corticosteroids therapy and interventional bronchoscopy treatment. Being reexamined after 2 months, she showed remissions of the tracheal lesions, negative smear sputum, weight gain and no cough. Conclusion: In endobronchial tuberculosis clinical and radiological features are non-specific, bronchoscopic evaluation of the bronchial tree being essential for the diagnosis. A late diagnosis might contribute to cicatricial stenosis and bronchiectasis despite the antituberculous therapy.

Keywords: endobronchial tuberculosis, bronchoscopy, wheezing

\section{Improved Lung Function and Life Quality for the Lymphangioleiomyomatosis Patients Treated with Rapamycin}

Neacşu G Gabriela Roxana Louisse', Preda E Andreea Maria', Tuţă R Mălina Denisa', Mihãilescu SM Claudia Ileana', MD Neacsu Anne Mary Denisse ${ }^{2}$

"Carol Davila" University of Medicine and Pharmacy Bucharest Romania, 2"Marius Nasta" Pneumoftiziology Institute, Bucharest, Romania

Background: Lymphangioleiomyomatosis (LAM) is a rare disease of unknown origin, which affects women in their reproductive period. It is characterised by non-neoplastic proliferation of atypical smooth muscle cells in the lung parenchyma, lymphatic vessels, mediastinal and abdominal lymph nodes. The most common presenting symptoms are recurrent pneumothorax, effort dyspnea, hemoptysis and chylothorax. Case presentation: A 47-year-old nonsmoker female, was admitted presenting dyspnea during rest, fatigue, which started six months ago, and right sided thoracic pains during the past three months. The physical exam revealed a poor general condition, a previously placed tube in the chest wall, iatrogenic subcutaneous emphysema affecting the anterior chest wall, diminished breathing sounds in the right lower lobe, limitation to the amplexation of the respiratory movements, 
cyanosis in the perioral area and fingers, persistent low oxygen saturation $\left(\mathrm{SO}_{2}=85 \%\right)$, with tachypnea and sinus tachycardia. Chest X-ray showed bilateral reticular and chystic opacities. A high-resolution computer tomography (HRCT) showed right apical pneumothorax, pulmonary fibrosis and also noted centrilobular emphysema, minimal right pleural effusion, mediastinal lymph nodes in Barety space. Lung function tests revealed severe mixed ventilatory dysfunction with a reduced total lung capacity, and a severely reduced diffusion capacity of carbon monoxide (DLCO= $5.34 \%$ ). Forced expiratory volume in the first second $\left(\mathrm{FEV}_{1}\right)$ was reduced with $32 \%$. Forced vital capacity $(\mathrm{FVC})=55.2 \%, \mathrm{FEV} 1 / \mathrm{FVC}$ ratio $=32.1 \%$. Diagnostic thoracentesis showed a chylous effusion. Clinical features, HRCT with chystic opacities and pulmonary fibrosis associated with chylothorax suggest the diagnosis of a severe form of lymphangioleiomyomatosis, with hypoxic respiratory failure. The therapy included Rapamycin $2 \mathrm{mg} /$ day, oxygen therapy and a lipid free diet which enhanced the pulmonary function tests. Conclusion: Rapamycin therapy (mTOR inhibitors) improves lung function tests and quality of life. In this case, we can observe a spectacular response: before treatment FVC was $55.2 \%$ and after a year of therapy it reached $88.8 \%$. Longterm outcome is unknown. Typically, the disease progresses to chronic respiratory failure and the survival expection is 8-10 years from diagnosis.

Keywords: lymphangioleiomyomatosis, HRCT, Rapamycin

\section{Myasthenia Gravis and Pregnancy}

Soare D.A. Diana Elena', Stanciu M Adela', Diana Alexandra I Savu', Dan-Nicolae M. D. Stoenescu', Lecturer Bohîlțea Roxana Elena'

"Carol Davila" University of Medicine and Pharmacy, Bucharest, Romania

Background: Myasthenia gravis is an autoimmune neuromuscular disorder characterized by the presence of nicotinic-receptor autoantibodies which reduce the number of these receptors within the muscular junction. It is twice as common among women at a young age than men, therefore it may occur during pregnancy. Case presentation: We report the case of a 20 year old pacient, 34 weeks pregnant, known with a generalized form of myasthenia gravis for 4 years who is admitted to our clinic for pregnancy monitoring. She also requires treatment for an urinary infection with Klebsiella which led to ureterohydronephrosis on the right kidney. This infection caused a myasthenic crisis before presentation. The patient is admitted with generalized muscle weakness, deglutition disorders and dyspnea. Clinical examination revealed general muscle hypotonia and hypokinesis and paraclinical examination showed a hemoglobin of $8.3 \mathrm{~g} / \mathrm{dl}$, erythrocyte medium volume dropping to $67.4 \mathrm{fl}$, a hematocrit of $28.3 \%$ and high white blood cell count but within the normal limits of pregnancy. The infection was treated with Meropenem (an antibiotic which is allowed both in pregnancy and myasthenia gravis) and the neuromuscular disease was treated with methylprednisolone and neostigmine. The pregnancy was carried to term and the pacient gave birth to a male baby with a weight of 2650 grams. Conclusion: Pregnancy in women with myasthenic disorder requires close monitoring, as it is very unpredictable. Concerning the mother, studies have shown that one third of the patients have an unfavorable evolution, one third notice an improvement of the disease and one third have a stationary course of the disease during pregnancy. Regarding the newborns, $21 \%$ present transitory neonatal myasthenia gravis.

Keywords: Myasthenia gravis, pregnancy

71. Particularities in Wolff-Parkinson-White Syndrome Diagnosis

Iulia-Madalina D Staicu', MD Anca Andreea Andronic', MD Claudiu Stoicescu'
7"Carol Davila" University of Medicine and Pharmacy, Bucharest, Romania

Background: Wolff-Parkinson-White (WPW) syndrome is defined as a congenital condition involving abnormal conductive cardiac tissue between the atria and the ventricles, different from the normal conduction pathway, of electrical impulses through the atrioventricular node. Through this fascicle, accesor name, electric impulse can conduct in the anterograde direction, retrograde direction, or both, causing this way the present of the atrioventricular reentrant tachycardia (AVRT). The diagnosis of WPW syndrome is based on EKG identification of preexcitation, which is associated with the appearance of the arrhythmia. WPW syndrome choice treatment is radiofrequency ablation of the accessory bead. Case presentation: I present the case of a 47 years old pacient, smoker, obase, hypertensive, who is present at the emergency room of Bucharest Emergency University Hospital for fast-paced palpitations, dyspnea and previous chest pain- 2 hours debut symptoms. His past medical history includes: HTAE neglected therapeutically and sporadically episodes of fast-paced, paroxysmal, self-limiting, uninvestigated palpitations. The electrocardiogram reveals tachyarrhythmia, narrow QRS, with diffuse ischemic changes, which is spontaneously converting to sinus rhythm, presenting HVS and intermittent preexcitation. The ecography in the emergency room immediately after remission of the arrhythmic episode reveals hypokinesia left ventricular diffusion, FEVS $45 \%$, concentric HVS, with further improvement during admission - FEVS $55 \%$ at discharge. Biologically there is an increase in Trop I (1.71 ng / ml), CKMB and NTproBNP. The diagnosis of complicated WPW syndrome with acute coronary syndrome and acute heart failure phenomena is established. Evolution during hospitalization was favorable, with no recurrence of arrhythmia, below the moderate dose of oral betablocant. It was programmed for radiofrequency ablation by the accessory beam. Conclusion: Possible complications of WPW syndrome are: acute coronary syndromes, acute heart failure or even sudden death. The recommendation ACC/AHA Guide 2015 for these patients is the radiofrequency ablation of the accessory bead, the drug being reserved only to patients who are not willing or refused to intervene. The specificity of the case is the increase of Troponin I to a not very high ventricular alloria ( $70 \mathrm{bpm}$ ), limiting the use of IC class antiarrhythmics to prevent recurrence of tachyarrhythmia.

\section{Keywords: syndrom, tachycardia, Wolff-Parkinson-White}

72. Predictors of Mortality in Critically III Cirrhotic Patients with Acute-On-Chronic Liver Failure Hospitalized in the Intensive Care Unit

Andrian S Titus ${ }^{1,2}$, Andronic M Daniela ${ }^{1,2}$, Trifan Anca ${ }^{1,2}$, Chiriac Stefan ${ }^{1,2}$, Professor Anca Trifan', Lecturer AnaMaria Singeap1, Assistant Lecturer Stefan Chiriac ${ }^{1}$ 1 "Grigore T. Popa" University of Medicine and Pharmacy, Iași, Romania, 2"St. Spiridon" Emergency Hospital laşi, Romania

Background: Liver cirrhosis (LC) has been associated with high mortality rates, especially in intensive care units. Although the natural progression of LC leads to decompensation and subsequently death, surprisingly, some patients develop organ failures more rapidly and have a worse prognosis than others. These observations led to the definition of a new concept, namely Acute-on-chronic liver failure (ACLF), a syndrome characterized by organ dysfunction and high mortality in decompensated cirrhotics. We aimed to assess the predictive factors for mortality in the intensive care unit for patients with ACLF. Materials and methods: We performed a retrospective study including cirrhotic patients hospitalized in the intensive care unit of "St. Spiridon" Emergency Hospital Iaşi, Romania, between march 2015- september 2017. Results: 72 patients were included, mostly men ( $72.2 \%)$, mean age $62 \pm 5.4$ years. The etiology of LC was alcohol ( $53 \%$ ), hepatitis C virus (HCV) $(33 \%)$, alcohol and HCV $(8 \%)$, alcohol and hepatitis B virus (HVB) $(3 \%)$, and HVB and hepatitis D virus in $3 \% .58(81 \%)$ 
died and the median survival was 4 (3-7) days. ACLF was diagnosed in $81 \%$, mostly ACLF grade 3 ( $83 \%$ ). Within the ACLF group, the death rate was $90 \%$ and ACLF grade 3 patients had the highest mortality (96\%). ACLF was a risk factor for death [OR=2.092, $\mathrm{Cl}(1.135-3.854), \mathrm{P}<0.001]$. In the ACLF group univariate analysis identified circulatory $[\mathrm{OR}=1.852, \mathrm{Cl} \quad(0.693-4.948), \mathrm{P}=0.034]$, coagulation $[O R=1.200, \mathrm{Cl}(1.037-1.389), P=0.013]$, and renal failure $[O R=1.385, \mathrm{Cl}(0.782-2.451), P=0.05]$ as risk factors for death; circulatory failure was also a mortality predictor in multivariate analysis $(P=0.045)$. Receiver- operating characteristic analysis showed good mortality predicting accuracy for Model for end-stage liver disease (MELD) and Chronic liver failure (CLIF) scores and moderate for Child-Pugh score (area under the curve 0.904, 0.897, 0.795, respectively). Conclusion: Mortality was high among critically-ill cirrhotic patients and even higher in LC patients with ACLF, MELD and CLIF scores could be used to accurately predict death in patients with ACLF hospitalized in the intensive care unit. Circulatory, renal and coagulation failures were predictors of mortality, therefore intensive treatment should be provided for these patients. As almost all cirrhotics with ACLF grade 3 died, intensive treatment could be futile and thus withheld for these patients.

Keywords: liver cirrhosis, acute-on-chronic liver failure (ACLF), mortality

\section{Sarcoidosis - A Multisystemic Disease}

Vulturar D Damiana-Maria', Taru L. Vlad', Rendler C Livia', Sîrbu D. Alexandru-Constantin', Lecturer Grad Simona "University of medicine and pharmacy "Iuliu Hațieganu" ClujNapoca

Background: Sarcoidosis is a chronic granulomatous inflammatory disease of unclear etiology. The characteristic histological finding of sarcoid granuloma consists of epithelioid cells, multinucleated cells without area of necrosis, mononuclear cells and CD4+ T cells with a few CD8+ T cells in the peripheral zone, unlike caseating granuloma of tuberculosis. Lung and intra-thoracic lymph node are the most commonly affected organs although any organs can be involved. The clinical course of sarcoidosis ranges from an indolent process to an acute self-limited process to a progressive disease with permanent organ damage. Case presentation: A 76 years old-woman complaining of dry cough, dyspnea, sweating, diarrhea, loss of appetite, fatigue and loss of weight (11 kilograms in 3-4 months) presented to the Pneumology department. The objective examination showed rhonchi in the middle third of the lung thoracic region. The thoracic radiography showed accentuated interstitial markings, the bronchoscopy showed a mucopurulent chronic bronchitis and the spirometry revealed an obstructive ventilation defect. Abdominal ultrasonography showed a nonhomogenous liver with a macro nodular eco structure, reason for why our patient was moved to the Gastroenterology department. The clinical findings included a pain in the right hypochondria, epigastrium and a hepatomegaly which was with an increased consistence of the liver and irregular edge. To exclude the causes of hepatomegaly, chronic diarrhea and of the anemia there were done more examinations. The CT examination revealed a chronic hepatopathy, adenopathies in the hepatic hill $(18 \mathrm{~mm})$, peri-celiac $(10 \mathrm{~mm})$, interaorto-cave $(13 \mathrm{~mm})$ and an inflammatory aspect. The result of the lower digestive endoscopy was a terminal ileitis and of the upper digestive endoscopy revealed an erosive pangastritis and congestive body. Liver biopsy procedure confirmed the diagnosis of sarcoidosis. The patient received combined oral cortico-therapy (Prednison 40mg/24h), immunosuppressive therapy (Imuran $150 \mathrm{mg} / 24 \mathrm{~h}$ ) and hepatoprotective drugs (Ursofalk $10-15 \mathrm{mg} / \mathrm{kgc} /$ day). After admission patient's condition worsened. She presented arthralgia, myalgia and diabetes. The treatment was changed with Plaquenil $(400 \mathrm{mg} / 24 \mathrm{~h})$, Metrotrexat $7,5 \mathrm{mg}$ and oral antidiabetics. Conclusion: The presented case is particularly distinctive in terms of diagnosis of exclusion- the symptoms were like in a malignant syndrome and also the exclusion of all the causes of a chronic liver disease. Furthermore sarcoidosis is a multisystemic disease.

Keywords: sarcoidosis, biopsy, multysistemic, malignant syndrome

\section{Severe Acute Colitis in Response to Bowel Preparation Prior to Elective Surgery}

Florea N Roxana',2, Buturoiu Monica Maria', Mitran G.S Raluca-Elena', MD Marina Ciochina², Lecturer Vasile Daniel Balaban1, MD Andreea Zoican², MD Georgiana Robu², Professor Mariana Jinga'

'"Carol Davila" University of Medicine and Pharmacy, Bucharest. Romania, ${ }^{\prime \prime}$ Dr. Carol Davila" Central Military Emergency University Hospital, Bucharest, Romania

Background: Severe colitis is a potentially life-threatening condition, which warrants prompt management. latrogenic severe left sided colitis, induced by gynecologic surgery preoperative enema, has been rarely reported. Case presentation: We report the case of a 45-years-old female, with unremarkable medical history, who was transferred to the Gastroenterology Department for severe lower abdominal pain and rectal bleeding that occurred immediately after she underwent a preoperative enema for pelvic reconstruction. Physical examination revealed abdominal tenderness in the lower left quadrant, with guarding and rebound tenderness. The patient reported profuse loose stools mixed with blood and mucus. Laboratory workup revealed significant biologic inflammatory syndrome (markedly elevated ESR, fibrinogen and C reactive protein), high leukocytosis with granulocytosis, hypoalbuminemia, mild normocytic anemia. Stool samples (for parasites, extended cultures and Clostridium difficile screen) were negative. We suspected iatrogenic colonic perforation but plain abdominal X-ray did not show pneumoperitoneum. A CT was performed which detected mural thickening of the sigmoid colon pericolonic fat stranding, paraaortic adenopathy and a small quantity of fluid in the Douglas pouch. On rectosigmoidoscopy the mucosa of the rectum and distal sigmoid colon showed severe edema and extended necrotic areas with submucosal hemorrhage, large ulcerations with mucopurulent exudate. The histopathology report revealed severe inflammation, granulation tissue and necrosis. A diagnosis of severe left-sided colitis was set. We considered bowel ischemia as an etiology, but angio CT did not reveal any arterial obstruction. Further tests were done, with no supporting evidence for other frequent etiologies of colitides. Treatment with combined broad spectrum antibiotics, corticoids, mesalazine, analgetics and low molecular weight heparin was started, with favourable clinical and biological evolution. On follow-up colonoscopy at two and four months, the bowel lesions markedly improved and the patient was symptom-free. Conclusion: Our case highlights a rare complication of a routine, frequently performed medical manuever, which can develop into a life-threatening condition. The diagnosis can be challenging because of the multitude of possible etiologies of acute colitis. In this case, the medical history plays a key role to set the right diagnosis.

Keywords: severe colitis, iatrogenic, enema, rectal bleeding, rectosigmoidoscopy

\section{Study of Major Complications in Liver Cirrhosis}

Mihai B Ruxandra', Starica V.M Alexandra', Miron R Mihnea', Iosep G Diana', Lecturer Gabriela Stefanescu' "Gr. T. Popa" University of Medicine and Pharmacy, lasi, Romania

Background: Liver cirrhosis is a severe disease, with many complications and a poor prognostic. In the last years the management of liver cirrhosis complications improved without a significantly decreasing of the mortality. The purpose of the study is the assesment of death causes in patients with liver cirrhosis. Materials and methods: We have studied death causes in patients with liver cirrhosis admitted in the Institute of Gastroenterology 
and Hepatology between 1st January 2016 and 1st January 2017. We have tried to highlight a possible correlation between etiology, complications, Child class, obesity, diabetes mellitus and the duration of the disease. Results: We have studied 730 patients; 28 deaths have been recorded $(3.89 \%$ ) (group A). The death causes, according to their frequency were: hepatic coma- 8 , haemorrhage shock induced by upper digestive haemorrhage (UDB)- 7 , hepatorenal syndrome (HRS)- 6, spontaneous bacterial peritonitis (SBP)5 , and two cases consisted of associated diseases. HRS and SBP were the complications with highest mortality $(80 \%$, respectively $76 \%$ ). Although UDB, compared to other complications was associated with a low mortality rate in itself, it was found as a risk factor in 18 of the deaths. The death rate was correlated with a long history of the disease, the male sex, Child class, obesity and type II diabetes mellitus, but was not correlated with etiology. Conclusion: Due to its complications liver cirrhosis is a high-risk mortality disease. SBP and HRS are the complications with the highest death risk, but in almost all cases UDB is the most important risk factor. Death risk is linked to the long history of the disease, its complications, and the liver potential assessed by Child score.

Keywords: liver cirrhosis, hepato-renal syndrome, upper digestive haemorrhage

76. The Correlations between iNOS-2087A>C Polymorhism and Acute Pancreatitis

Marcu A Andreea-Ariana', Professor Saftoiu Adrian', Assistant Lecturer Dr Padureanu Vlad

'University of Medicine and Pharmacy of Craiova, Romania

Background: Acute pancreatitis is a pathological condition localized in the pancreas gland involving a systemic inflammatory response. It is a pathology widely encountered recently, evolving with increasing incidences that vary from a continent to another, depending on ethnicity, genetic predisposition and diet. Consequently it is very important to know exactly which mechanisms are involved in their pathophysiology. The objective is to investigate whether there is a possible correlation between single nucleotide polymorphisms (SNP) of the inducible nitric oxide syntethase (iNOS) and acute pancreatitis, using genetic analysis. Materials and methods: Biological samples with peripheral blood were obtained from patients admitted on Gastroenterology, 1st Surgery and 2nd Surgery clinics of the Emergency County Hospital of Craiova, between March 2014September 2015. Genomic DNA was extracted from blood samples from patients diagnosed with acute pancreatitis $(n=168)$ and healthy subjects without inflammatory pathology $(n=232)$. inOS$2087 \mathrm{~A}>\mathrm{G}$ polymorphism was genotyped and allelic variants were identified by the technique Real Time PCR with TaqMan probes. It was also evaluated the association of the genetic polymorphism studied based on the clinical and pathological data of the patients. Results: The polymorphism we studied was in Hardy-Weinberg equilibrium for both acute pancreatitis and healthy control groups. Between the investigated polymorphism and the risk of developing acute pancreatitis was found a slight association but insignificant statistically. Conclusion: In the studied group, the risk of developing acute pancreatitis is not increased by the presence of the polymorphism iNOS - 2087> G but instead is increased by alcohol.

Keywords: iNOS - 2087> G polymorphism, acute pancreatitis , genetic analysis

77. The Evolution of a Measles Case Originating from a Family Epidemic Outbreak

Luca D Stefana', Avadanei N Andrei Nicolae', Stafie L Ingrid', Froicu D Eliza Maria', Associate Professor Carmen Manciuc $^{1}$

"Grigore T. Popa" University of Medicine and Pharmacy, lasi, Romania
Background: Measles is an eruptive, very contagious disease, characterized by fever, cough, coryza, conjunctivitis, followed by an erythematous maculopapular rash and a pathognomonic enanthem (Koplik spots). In the past 2 years, the incidence of the disease has visibly grown, with Romania being the main outburst of measles in Europe (5300 cases and 24 deaths confirmed in 2017). The evolution of this condition is positive in the absence of complications, hospitalization being recommended in severe and complex forms. Prophylaxis is based on active immunization with two doses of vaccine or passive immunization with standard Gamma globulin. We are presenting a special case, coming from a family epidemic outbreak, developed in the absence of vaccination. Case presentation: A 4 years old female patient, from the rural area, is clinically examined for fever, cough, coryza. The anamnesis reveals that the child comes from a familial measles outbreak and has no significant pathological history. In evolution, the general condition worsens with persistence of fever, the presence of pulmonary condensation syndrome and the occurrence of maculopapular rash after 72 hours. The previous signs accompanied by the Koplik sign at the level of the jugal mucosa raises suspicion of measles. It is noted that the child is unvaccinated against the disease. Non-specific tests for diagnosis showed normal blood counts with lymphocytosis and radiological conformation of pneumonia. Positive diagnosis has been confirmed by the presence of anti-measles IgM antibodies. Patient's condition was complicated with left lobar pneumonia by bacterial superinfection, for which she received antibiotic and symptomatic treatment. The evolution of the subject was good under treatment, requiring 7 days of admission. Conclusion: The particularity of this case is represented by the familial epidemic outbreak and the occurrence of the Koplik sign simultaneously with the eruption. Further, the case highlights the danger of nonvaccination against measles in children and the need for compulsory hospitalization if complications occur, noticing that the clinical and epidemiological profile of measles has changed lately by the increasing number of cases.

Keywords: measles, family epidemic outbreak, Koplik sign, nonvaccination

\section{The Influence of the Risk Factors for a Tricoronarian Patient}

Vreme E Otilia Flaviana', Ilinca C Diana-Andreea', Valentina Paraschiva M Huțanu', Gabriel-Valentin MA Tanase', MD PhD Teodor Vasilcu', MD PhD Ana Nicolae ${ }^{2}$, MD PhD Iris Bararu', MD PhD Radu Gavril', MD PhD Cristina Prisacariu', MD PhD Catalina Georgescu-Arsenescu'

"Crigore T. Popa" University of Medicine and Pharmacy, lassi, Romania, "2"St. Spiridon" Emergency Hospital Iassi, Romania

Background: The prevalence of coronary heart diseases is drastically increasing due to the risk generated by inadequate lifestyle choices in the general population. Considering that coronary heart disease is one of the main causes of morbidity and mortality in adults, the risk factors that lead to any cardiac event are known to have a massive influence in the patient evolution and the treatment must be conducted accordingly. Case presentation: We present a case of a 66-year-old patient, with a history of cardiovascular risk factors (obesity, diabetis, long-term hypertension), who has undergone in 2009 a percutaneus transluminal coronary angioplasty (PTCA) on the left anterior descendent artery (LAD) and right coronary artery (RCA), implantation of bare-metal stent (BMS) on the left circumflex artery $(\mathrm{LCX})$, followed by a triple bypass in the same year, and a drug-eluting stent (DES) implantation on RCA in 2014, is admmited in our hospital for recurent anginous episodes. The patient was admitted with $150 / 80 \mathrm{mmHg}$ blood pressure and a heart rate of 80 beats / minute, a systolic heart murmur (4/6) located in the aortic valve area which radiates to both carotid arteries. The electrocardiogram showed a $1 \mathrm{~mm}$ ST depression in $\mathrm{D}_{1}, \mathrm{aVL}, \mathrm{V}_{5}$, V6. The echocardiography has revealed a concentric left ventricular 
hypertrophy with preserved systolic function, an intense calcified aortic valve, mild mitral regurgitation. Further analysis also revealed an inflammatory syndrome, hyperglycemia and renal disfunction. The coronarography revealed $99 \%$ LAD I stenosis and chronic LAD II occlusion, 90\% DG I, 99\% LCX I, a permeable LCX II stent, 99\% LCX III, 95\% OM I, permeable RCA stents. The flux was satisfactory reestablished through a PTCA, using balloon angioplasty for distal LCX and OM I and a stent on LCX I. Conclusion: Our patient symptoms improved, having good posttreatment evolution and was scheduled for a back-up check of her ambulatory blood pressure measurement (ABPM), echocardiography and a Holter monitoring to prevent a possible relapse. Even though the patient received the appropriate pharmacological and interventional treatment, major lifestyle changes also play an important role in the holistic treatment of cardiovascular patients.

Keywords: tricoronarian, risk factors, cardiovascular

Gastrointestinal Manifestations of Systemic Sclerosis Spoială L Maria', Associate Professor Svetlana Agachi' 'Nicolae Testemitzanu State University of Medicine and Pharmacy, Chişinău, Republic of Moldova

Background: Systemic sclerosis (SSC) is an autoimmune, multisystemic disease of unknown cause, characterized by diffuse fibrosis, degenerative changes, and vascular abnormalities in the skin, joints, and internal organs (especially the esophagus, lower gastrointestinal (GI) tract, lungs, heart, and kidneys). After the skin, the most commonly involved system is the GI tract. Materials and methods: We performed a retrospective study, in which were included 80 patients diagnosed with SSC who were hospitalized in the Rheumatology and Arthrology departments of Republican Clinical Hospital, during the years 2014-2015. Results: The average age of the patients with SSC was $52,82 \pm 19$ (range 18-78) years and the average duration of disease- 12,4 years. Distributing patients by sex established the predominance of women in comparison with men: $77(96.25 \%)$ women vs $3(3.75 \%)$ men. The average activity of SSC (EUSTAR)- $3.85 \pm 1,5$. Castrointestinal manifestations of systemic sclerosis were found in $98,75 \%$ (79 patients of 80 ), including: microstomia- $32(40 \%)$, xerostomia- 30 $(37,5 \%)$, secondary Sjogren's syndrome- $10(12.5 \%)$, dysphagia- 64 $(80 \%)$, esophageal dysmotility- $36(45 \%)$, esophageal reflux- 16 $(20 \%)$, vomiting- $2(2,5 \%)$, nausea- $3(3,75)$, abdominal pain- 20 $(25 \%)$, bloating- $8(10 \%)$, constipation- $11(13,75 \%)$, diarrhea- 2 $(2,5 \%)$, loss of appetite- $8(10 \%)$, weight loss- $3(3,75 \%)$, Gl bleeding- $1(1,25 \%)$, malabsorbtion- $1(1,25 \%)$, prim biliary sclerosis- 1 (1,25\%). Conclusion: Almost every part of the $\mathrm{Gl}$ tract can be involved. The esophagus is the most commonly involved organ of the $\mathrm{Gl}$ tract in SSc. Malnutrition, diarrhea, and constipation are some Gl complications that can stem from scleroderma, and they contribute considerably to the impairment in quality of life. It is therefore particularly important to identify, monitor and manage these patients carefully, with a view to minimise further degeneration and maximise quality of life.

Keywords: systemic sclerosis, digestive involvement, scleroderma

\section{0. \\ A Rare Case of Ventricular Tachycardia in a Pregnant Patient}

Popovici M Catalina Gabriela', Serban A Ioana Adriana', Ioanid N Alexandra Nicoleta', Rusu V Ioana', Assistant Lecturer Sutu Cristina Ramona ${ }^{2}$

"Grigore T. Popa" University of Medicine and Pharmacy, Iasi, Romania, 20vidius University, Faculty of Medicine, Constanţa Romania

Background: Arrhythmias represent some of the most common cardiac diseases. In the case of pregnant patients (with no associated comorbidities or eclampsia) these pathologies are of great interest, due to the fact that in the second and third trimester of pregnancy they are usually asymptomatic. Therefore, these abnormal modifications can easily lead to the hemodynamic destabilisation of the mother, along with fetal suffering, if they are not discovered in time. Case presentation: We will present the case of a female patient (28 years old), primiparous ( 32 weeks), who requested an emergency medical team (EMT) due to palpitations which debuted 2 weeks ago, but worsened in the last 24 hours. The patient did not present premature contractions or loss of amniotic fluid; however, after being connected to a vita sign monitor, Ventricular Tachycardia (unsustained) with Pulse (Cardiac Rate 120bpm, Sp02 95\%, Arterial Pressure 138/80) was discovered. The patient was administered Xylin (IV pump $1 \mathrm{mg} / \mathrm{kg} / \mathrm{h}$ ) and Xylin bolus (50mg). Upon arrival in the ECAA department a Complete Blood Cell Count and Biochemical Tests were requested. After the electrocardiographical (ECG) confirmation of the diagnosis, the patient was consulted by a Cardiologist and a Gynecologist before being admitted in the Cardiology Department. During the first day, the treatment consisted of Beta-blockers (Betalok 5omg/day) and Acetyl Salicylic Acid (Aspenter). The dose of Beta-blockers was doubled during the second day having taken into account the results of ECG Holter Test, which showed the persistence of the Tachyarrhythmias. After the adjustment of the dosage, the episodes of Ventricular Tachycardia decreased in number until they disappeared. During the entire treatment, the patient was also closely monitored by the Obstetrics-Gynecology Department. Conclusion: Ventricular Tachycardia represents a major risk factor for hemodynamic instability, with great implications for pregnancies. Correct identification of gynecological emergencies caused by arrhythmias, and early treatment of these pathologies, leads to avoiding some limit situations, such as performing an Emergency Caesarian Section, a case which would raise ethic and medical problems.

Keywords: pregnancy, ventricular tachycardia, arrhythmias, betablockers

81. The Screening of Antibiotic Resistance Development in Pseudomonas Aeruginosa in Intensive Care Unit of the University Clinical Hospital, Ternopil, in 2013-2016 Pokryshko PO Alina', Appiah JA Emmanuel², Salem Mohammed Abdelgawwad MA ahmed ${ }^{2}$, Associate Professor Elena Pokryshko3

'Zaporozhye State Medical University, Ukraine, Intensive Care Unit of the University Clinical Hospital, Ternopil, ${ }^{2}$ Ternopil State Medical University, 3Department of Microbiology, Virology and Immunology at Ternopil State Medical University

Background: Nowadays infections caused by non-fermenting gramnegative rods, in particular Pseudomonas aeruginosa, is one of the actual and serious problems in hospitals. In most cases, these bacteria have been multidrug-resistant and the elimination of Pseudomonas aeruginosa in patients with infection is very difficult. The purpose of researches was to trace the dynamics of antibiotic resistance development of $P$. aeruginosa strains isolated from patients of intensive care unit of the University Clinical Hospital, Ternopil in 2013-2016. Materials and methods: Microorganisms were isolated from clinical specimens, such as sputum, tracheobronchial aspirate, urine, swabs from infected wounds. All cultures were isolated according to standard laboratory protocol. Their identification and determination of susceptibility to antibiotics were tested by an automated microbiology system Vitek Compact-2 (bioMérieux). The study was based on the regular screening of the bacterial susceptibility to antibiotic during 4 years. The application package "Statistica 6.0" was used in the data processing methods. Results: 120 Pseudomonas aeruginosa clinical isolates were tested: 25 cultures (in 2013), 39 (2014), 24 (2015), 32 (2016). Cephalosporins (cefoperazone, ceftazidime, cefepime) were becoming less effective every year. In 2016, just $10 \%$ of Pseudomonas aeruginosa isolates were susceptible to this group of antibiotics. Among carbapenems, imipenem was the most effective: $31.8 \%$ of Pseudomonas aeruginosa isolates retained susceptibility to it in 
2016. At the same time, meropenem lost its effectiveness every year more and more: in 2013, 21.4\% of Pseudomonas aeruginosa isolates were susceptible to this antibiotic, in 2016 - only $5.9 \%$. Over the past years, the increasing resistance to fluoroquinolones in Pseudomonas aeruginosa isolates was detected. Only piperacillin/ tazobactam had remained effective against Pseudomonas aeruginosa in almost half of the cases (2014 $56.7 \%$, in $2015-45.2 \%$ ). Conclusion: The screening had shown that the resistance to the main groups of antibiotics in Pseudomonas aeruginosa isolates increased over last years. First bacteria lose susceptibility to most often used antimicrobial treatment medicines: cephalosporins, fluoroquinolones and carbapenems.

Keywords: Pseudomonas aeruginosa, multi drug-resistant, antimicrobial treatment medicines

82. The Value of Impedance Cardiography and Oxygen Pulse During Exercise Testing in Patients with Hypertrophic Cardiomyopathy

Vuijk A. David', Associate Professor Francois Haddad², MD Kegan James Moneghettí2, Academician Jeffrey W. Christtle $^{2}$

'Leiden University, Stanford Cardiologic Institute, ${ }^{2}$ Stanford Cardiovascular Institute

Background: Monitoring exercise response during cardiopulmonary exercise testing (CPX) has always been important in heart failure patients. However, during routine exercise testing, cardiac output (CO) and stroke volume (SV) response is not routinely measured due to impractical techniques. New methods for monitoring hemodynamic values would add incremental value. In recent years impedance cardiography (ICG) has emerged as a non invasive way to do so. In order to better understand exercise response in heart failure patients we sought to explore the relationship of oxygenpulse (02 pulse) with SV and the implication for prognostication in hypertrophic cardiomyopathy (HCM). Materials and methods: We selected patients with HCM as defined by international guidelines who underwent exercise testing with CPX and ICG. SV was calculated by echocardiography at rest and immediately postexercise using the area length method. Patients were followed for a median of 4.5 years for the composite endpoints of heart failure deterioration, left ventricular device insertion, heart transplant or death. Results: A total of 94 patients with HCM were included, $67 \%$ male, mean age $51 \pm 14$ and BMI $28.3 \pm 5.2 \mathrm{~kg} / \mathrm{m2}$. There was a moderate correlation between 02 pulse and SV on echocardiography, $(r=0.39, p<0.01)$, which was greater when measured by ICG $(r=0.65, p<0.01)$. In a multivariate model with 02 pulse as the dependent variable, $62 \%$ of its variation was explained by age, sex and peak SV by ICG. Exploratory outcome analyses with cox hazard ratios suggested $\mathrm{V}_{2} 2$ peak along with VE/VCO2 slope to be associated with outcome ( $x 2=15, p<0.01)$. Conclusion: SV as assessed by ICG appears to have a significant role in the variation of $\mathrm{O}_{2}$ pulse even more so than echocardiography. Despite this finding in $\mathrm{HCM}$, among CPX variables V02peak and VE/VCO2 slope appear to play the strongest role in outcome prediction.

Keywords: cardiopulmonary exercise testing, hypertrophic cardiomyopathy, heart failure, impedance cardiography, echocardiography, cardiac output, stroke volume, new techniques, prognosis

\section{When Guidelines Are Not Enough}

Nitu-Preda T. Ionela', Assistant Lecturer Alexandru Ioan Deaconu ${ }^{1,2}$

"Carol Davila", University of Medicine and Pharmacy, Bucharest, Romania, ${ }^{2}$ Clinical Emergency Hospital of Bucharest, Romania

Background: Thrombolytic treatment of acute pulmonary embolism restores pulmonary perfusion more rapidly than anticoagulation with unfractionated heparin alone. Guidelines recommend systemic thrombolysis for high mortality risk pulmonary embolism and should be considered for patients with intermediate-high mortality risk, if clinical signs of hemodynamic decompensation appear. Case presentation: A 73-year-old female patient, with hypertension, dyslipidemia and diabetes presented at emergency room with severe dyspnea, lipothymia and tiredness. Patient is a non-smoker and has no relevant family history. Her blood pressure was $140 / 60 \mathrm{mmHg}$, heart rate was $100 / \mathrm{min}, \mathrm{SaO} 2=96 \%$ with oxygen supply, respiratory rate $34 / \mathrm{min}$. On physical examination she had cold, cyanotic teguments, without edema. Electrocardiography showed tachycardia, $\mathrm{S}_{1} \mathrm{Q}_{3} \mathrm{~T}_{3}$ pattern, $\mathrm{T}$ inverted in $V_{1}-V_{5}$ without other changes of repolarization. Transthoracic echocardiography revealed dilated right cavities, flattened interventricular septum, with paradoxical movement, absence of inspiratory collapse of inferior vena cava, pulmonary hypertension. Computed tomography showed occlusive thrombus in the left and right pulmonary artery, extending to the lobar and segmental branches. Her clinical status became worse. Dyspnea aggravated, blood pressure became $110 / 65 \mathrm{mmHg}$, heart rate $111 / \mathrm{min}$, Sat02 $=86 \%$ with oxygen supply. The patient was a high risk PESI class IV. She had not absolute or relative contraindications for thrombolysis. She was successfully treated with alteplase and her clinical and paraclinical parameters improved. Conclusion: Thrombolytic treatment can be life saving in patients with pulmonary embolism at intermediate-high mortality risk. In pulmonary embolism, systolic blood pressure drop by 30 $\mathrm{mmHg}$ with other signs of severity like sever dyspnea, right ventricular dysfunction and dilatation, can lead to death. Thrombolytic treatment saved the life of our patient.

Keywords: pulmonary embolism, thrombolysis, intermediate-high mortality risk, alteplase

84. When the Unexpected Happens, Adapt Approaches: A Rare Case of Transseptal Cardiac Resynchronization Therapy Defibrilator Implantation

Cosoreanu G Vlad', Mihai I Catalina', Ilie T Maria-Teodora', Cristian I Gherghe1, Assistant Lecturer Dr. Stefan Bogdan ${ }^{2}$

"Carol Davila", University of Medicine and Pharmacy, Bucharest, Romania, ${ }^{2}$ Clinical Emergency Hospital of Bucharest, Romania

Background: Cardiac Resynchronization Therapy-Defibrillator (CRTD) is an electrical stimulation device consisting of three leads through which the subcutaneous generator delivers tiny amounts of electrical energy to the heart, thus restoring the normal timing of the myocardial depolarization and contraction, causing both ventricles to pump together more efficiently. The three leads are inserted through the subclavian or femoral vein, each one of them being guided to a different site of the heart: one in the right atrium, one in the right ventricle, and the last one takes the course of the main epicardial vein that drains the left ventricle. This way, two of the leads are placed intracavitary, and the third one is fixed intravenously. In the recent years, CRT-D became largely used in patients with heart failure caused by complex arrhythmias which also pose a great risk of sudden arrhythmic death. Considering the multiple ways an electrical dysfunction can lead to a decrease in the heart's function as a pump, CRD-T may become a long-term solution, as a versatile and adaptable device. Case presentation: A 72 years old male patient, known with left bundle branch block, paroxysmal atrial fibrillation, dilative cardiomyopathy, with a Left Ventricle Ejection Fraction (LVEF) of $20 \%$ and permeable coronary arteries, is subject to CRT-D. After 18 months, the patient presents to the hospital with the venous lead detached from the initial site. Repositioning of the lead is performed, but the ventricular response is absent. Another approach is chosen. This time, the lead is passed from the right to the left atrium, through the interatrial septum by septotomy, and then intracavitary, in the left ventricle. The treatment is efficient and the heart function ameliorates. Conclusion: The LVEF of $20 \%$ is mainly caused by the inefficient sequence of the left ventricle's depolarization and contraction. Improper fixation to the walls of the vein is probably the cause of detachment. Thrombosis and 
iritation of the myocardium may be the cause of no electrical and contractive ventricular response after repositioning the lead. Transseptal approach to the left ventricle imposes lifelong anticoagulant therapy, counting as a major disadvantage.

Keywords: heart failure, cardiac resynchronization therapydefibrillator, cardiac electric stimulation

85. Evaluation of Cardiovascular Outcome in Patients with Chronic Heart Failure Using Popmètre $₫$

Tarhini H Mariam', F Teodor ${ }^{1,2}$, Belciu N Diana ${ }^{1,3}$, Ezatullah Dr.Abdul Rehman ${ }^{3}$, Lecturer Camelia Diaconu',

"Carol Davila", University of Medicine and Pharmacy, Bucharest, Romania, 2"Marius Nasta" Institute of Pneumology, Bucharest, Romania, 3 Clinical Emergency Hospital of Bucharest, Romania

Background: In recent years, pulse wave velocity (PWV) has been the "golden standard" for determining arterial stiffness. Heart failure patients may have an increased arterial stiffness, that may predict a worse prognosis. The aim of this study was to evaluate the utility of measuring the PWV with the p0pmètre ${ }^{\circledR}$ device in patients with chronic heart failure, in order to predict the cardiovascular outcome of these patients. Materials and methods: The study group consisted of patients with chronic heart failure admitted to the Clinical Emergency Hospital of Bucharest, between January-September 2017. In these patients, demographic, clinical and paraclinical data were collected and arterial stiffness was estimated by measuring the pulse wave velocity using the popmètre ${ }^{\circledR}$ device. Measurement was performed using two transducers, which estimated the finger-toe pulse wave velocity (ft-PWV), taking into account the age and height of the patient. Results: The descriptive study included 45 patients with chronic heart failure. Distribution of patients by gender: $55.56 \%$ women and $44.44 \%$ men. The mean age of the study group was $70.97 \pm 11.64$ years. The patients with heart failure had the following distribution of NYHA class: $44.44 \%$ NYHA class I, $45 \%$ NYHA class II, $25 \%$ NYHA class III, $30 \%$ NYHA class IV. $40 \%$ of patients with heart failure presented left ventricular hypertrophy at echocardiography, $33.33 \%$ diastolic dysfunction and $42.22 \%$ ischemic coronary disease. The distribution of major comorbidities in the group of study was: $55.56 \%$ dyslipidemia, $40 \%$ type II diabetes mellitus, $48.89 \%$ chronic kidney disease, $37.78 \%$ hepatic steatosis, and $17.78 \%$ had chronic obstructive pulmonary disease. $77.78 \%$ of the patients were smokers and $35.56 \%$ were obese. $37.78 \%$ of the patients had normal values of PWV scores, $15.56 \%$ intermediate values, and $46.6 \%$ high values. Conclusion: Patients in our study group showed an increased arterial stiffness, assessed by measuring ft-PWV with the p0pmètre ${ }^{\circledR}$ device. There was a significant correlation between increased arterial stiffness and a more severe NYHA class. Also, we have found a correlation between increased arterial stiffness and the presence of diabetes mellitus, chronic kidney disease as well as smoking history. Therefore, popmètre ${ }^{\circledR}$ can be used to monitor disease progression and to predict cardiovascular outcome.

Keywords: heart failure, cardiovascular risk, arterial stiffness, pulse wave velocity

86. Fecal Microbiota Transplantation for Treatment of Active Ulcerative Colitis

Tusa G Cristina Maria', Turac I János-Robert'', Dr. Lidia Ciobanu?

"Iuliu-Hațieganu" Univeristy of Medicine and Pharmacy, ClujNapoca, Romania

Background: Ulcerative Colitis (UC) is a chronic inflammatory disorder of the colorectum that has a peak incidence in early adulthood. The etiology of the disease is unknown, but it is thought to arise from an aberrant immune response to a change in colonic environment in a genetically susceptible individual. Current treatment modalities center on the modulation of the immune system but are limited by side effects. Recent trials suggest that Fecal Microbiota Transplantation
(FMT) is a promising treatment to induce remission in UC. Case presentation: A 31-year-old male, was admitted in 2012 with bloody diarrhea, having 8 to 10 stools per day. On colonoscopic examination, erythematous mucosa with multiple ulcerations were observed and biopsies were taken. Results were suggestive for UC, for which he started treatment with Infliximab and cortisone. Between 2012 and 2014 the patient had recurrent infections with Clostridium difficile, under treatment with Vancomycin. In October 2014, the first FMT was performed and successfully treated both antibiotic-resistant Clostridium difficile colitis and UC symptoms. In 2015 and 2017 two other FMTs were performed for keeping the UC symptoms under control. Currently the patient is under clinical but not endoscopic remission, having a Mayo score of 3 . He follows a treatment plan with Adalimumab and a strict diet, which play a role in maintaining the UC under control. Conclusion: The interaction between the intestinal microorganisms and an altered immune system in a susceptible individual is suspected to be central to the development of inflammatory bowel disease. We report a case of a patient with UC with recurrent Clostridium difficile infection, successfully treated by FMT. Beside the remission of the infection, FMT seems beneficial and safe for treatment of active UC, with temporary relief of the symptomatology. Both changes in diet and life-style contributed to maintain the remission of the disease.

Keywords: ulcerative colitis, inflammatory bowel disease, fecal microbiota transplant, Clostridium difficile, gut microbiota

\section{Follow Your Heart - Spy Out the Unthinkable}

Ciobanu G Georgiana', Lecturer Adrian Buzea², Assistant Lecturer Alexandru Deutsch², Professor Cheorghe Andrei Dan $^{2}$, MD PhD Istvan Neuhoff ${ }^{2}$

"Carol Davila", University of Medicine and Pharmacy, Bucharest Romania, ${ }^{2}$ Colentina Clinical Hospital Bucharest, Romania

Background: Atrioventricular conduction disorders are unpredictable entities, prone to develop life-threatening complications, by rhythm degeneration. Acute onset of symptoms requires precise diagnosis and prompt treatment. Case presentation: A 68-year-old female presented with asthenia and dyspnea, aggravated in the past week. Patient's history includes hypertension, ischemic stroke, type 2 diabetes. In the last six months she was diagnosed with type II atrioventricular (AV) block. She was bradycardic (50 bpm), with severe hypertension $(B P=200 / 90 \mathrm{mmHg})$ and hypoxic (02Sat $=87 \%$ spontaneously). The ECG confirmed type II AV block. Physical examination revealed signs of systemic congestion and pulmonary crackles. Lab tests showed hyperglycemia, hyperuricemia, elevated NT proBNP and positive D-dimer test. Chest radiography described cardiomegaly, enlarged pulmonary artery and a triangle-shaped opacity in the right lung. Echocardiography revealed dilated chambers, pulmonary hypertension and a reduced left ventricle ejection fraction (LVEF). The computerized tomography ruled out a pulmonary embolism, but revealed a renal tumoral mass and mediastinal adenopathies. She later developed type III AV block complicated with torsade des pointes. She was successfully resuscitated and a permanent cardiac pacemaker was implanted. The patient's hospital course was complicated by two episodes of acute pulmonary edema precipitated by hypertensive crisis. The patient had a significant alteration of the cardiac substrate and heart failure. This was explained not only by uncontrolled hypertension, but also by the probable ischemic heart disease, sustained by the presence of cardiovascular risk factors and previous stroke. In the acute setting the previous stable AV conduction disturbance needed continuous monitoring as it could have aggravated anytime. In our case, renal function changes due to newly diagnosed renal tumor were a plausible contributing factor for both hypertensive crisis and flash pulmonary edema. Conclusion: Even when obvious factors could explain a patient's condition, a thorough evaluation is essential to establish a complete diagnosis and achieve proper care. In acute settings a stable arrhythmia could aggravate into a lifethreatening condition. Secondary causes should be considered in the presence of difficult to control hypertension. 
Keywords: atrioventricular block, renal tumor, torsade des pointes, heart failure

88. Frequency of EGFR Mutations in Patients with Non-Small Cell Lung Carcinomas (NSCLC) from Different Samples and Centers - Retrospective Study

Kremenović LjK Stefan', Goran1, Baros Ilija', Associate Professor Ljiljana Tadić-Latinović'

'Medical faculty, University of Banja Luka, Banja Luka, Republic of Srpska, Bosnia and Herzegovina

Background: Non-small cell lung cancer (NSCLC) makes $85 \%$ of all lung cancers. The prognosis of metastatic non-small cell lung cancer still remains very poor. The detection of activating mutations for the epidermal growth factor receptor (EGFR) has become a standard biomarker for selecting patients for tyrosine kinase inhibitors (TKI) therapy. The aim of the paper is a retrospective analysis of the frequency of EGFR mutations in patients with non-small cell lung cancer. Materials and methods: At the Institute of Pathology of the University Clinical Center of Republic of Srpska in the period from 01.01.2016 until 31.12.2016 were analyzed samples of 236 patients with non-small cell lung cancer from Bosnia and Herzegovina. The tests were performed on samples obtained by surgical excision of primary tumor and metastases, fine bioptic samples and cytological samples. The histological type of tumor was determined on haematoxylin and eosin (HE) colored samples. In cases where it was not possible to make the diagnosis on HE colored samples, a panel of recommended antibodies (TTF $1, \mathrm{CK}_{7}, \mathrm{CK}_{5} / 6$ and / or p63, napsin A) was used for immunophenotyping of non-small cell lung cancer. Molecular testing was done with PCR method in real time with Roche Cobas reagents. The analysis of the results was done automatically on Cobas 4800 software with Cobas z 480 analyzer instrument. Results: 139 men and 97 women aged between 40 and 81 years were examined with a median of 62.92. Activating EGFR mutations were detected in $9.75 \%$ of patients. Mutations were more frequent in women $(6.78 \%)$ than in men $(2.97 \%)$. Deletion on Exon 19 was present in $56.52 \%$ of cases, and mutation on Exon 21 (L858R) was confirmed in $34.78 \%$ of cases. Insertion on Exon 20 was confirmed at $4.35 \%$. Conclusion: The frequency of EGFR mutations is lower in relation to literature data. In our study, better results were obtained by testing on samples obtained by biopsy compared to other types of samples.

Keywords: non-small cell lung cancer (NSCLC), EGFR mutations, EGFR testing, tyrosine kinase inhibitors (TKI)

\section{Gastric Lymphoma in a Young Patient}

Taru L. Vlad', Vulturar D Damiana-Mariaı, Nemeş G Cristina', Sîrbu D. Alexandru-Constantin', Lecturer Simona Grad $^{2}$

I"Iuliu-Hațieganu" Univeristy of Medicine and Pharmacy, ClujNapoca, Romania, ${ }^{2}$ Gastroenterology department, Clinical County Hospital "Medicala II", Cluj-Napoca, Romania

Background: Gastrointestinal tract is the most common extranodal site involved by lymphoma with the majority being non-Hodgkin type. Gastric lymphoma is responsible for $3-6 \%$ of the gastric cancers. $95 \%$ of them are non-Hodgkin and the most prevalent are B type and MALT lymphomas [4]. Over $90 \%$ of the patients with gastric lymphoma are infected with Helycobacter pylori (HP). Case presentation: A 23-year-old man presented with epigastric pain, vomiting aliments (2-3/day), epigastric plenitude and a downward weight curve of $10 \mathrm{~kg}$ in the previous month. Ceneral examination revealed a $B M I=13 \mathrm{~kg} / \mathrm{m} 2$, pale, dehydrated tegument. Abdomen examination did not show any signs of pain at palpation, but the patient presented clapotage. The clinical diagnosis included gastric evacuation insufficiency syndrome and alarm symptoms. Laboratory tests at presentation revealed high levels of LDH, Amylose, seric urea, hyposodemia, hypocloremia. Abdominal ultrasound showed a thickened gastric wall, a distended stomach with abundant gastric content. Endoscopy showed an infiltrative, stiff mass which could not be passed by. This mass was friable and bleeding when the biopsy was performed. Then, an ecoendoscopy was performed and a thoraco-abdominal TC which concluded the aspect of a T4NoMX gastric lymphoma. To confirm the diagnosis a laparoscopy was performed. Patient underwent treatment with Clorambucil, Cyclophosphamide and Rituximab since 2014. Conclusion: The particularities of this case reside in the discrepancy between the state of the disease and the late onset of symptoms. Another very important aspect of this case is the young age of the patient which is exceptional in the literature.

Keywords: gastric lymphoma, ecoendoscopy, Clorambucil, Cyclophosphamide, Rituximab

90. Interdependence Between Disease Activity, Quality of Life and Satisfaction in Patients with Idiopathic Inflammatory Myopathies

Loghin-Oprea V Natalia', Vetrila Snejana', Mazur-Nicorici Lucia', Salaru Virginia', MD Mazur Minodora', MD Cebanu Mariana'

"Nicolae Testemiţanu" State University of Medicine and Pharmacy, Chişinău, Republic of Moldova

Background: The matter of idiopathic inflammatory myopathies (IIM) - a group of diseases characterized by skeletal muscles asthenia, resides in evolution with flares and remissions that leads in hospitalizations. The aim: to study the correlation between disease activity, quality of life and satisfaction in myopathies patients. Materials and methods: We performed a cross-sectional study from August 2016-August 2017. The patients included in the study fulfilled the Bohan and Peter criteria for IIM. The collected information was about demographic data, clinical and laboratory findings. Disease activity was determined at the time of assessment by patient and doctor global assessment, respective PGA/MDGA, using a $100 \mathrm{~mm}$ VAS. The patient's satisfaction was assessed by self-administered Patient Satisfaction Questionnaire (PSQ III), which is a 50-item tool, covering 7 domains, and quality of life by SF-8 questionnaire for 8 domains and two components: mental (MCS) and physical (PCS). Results: There were 68 patients included in the study, of them 44 females and 24 males with a mean age $53.1 \pm 12.5$ (range 25-69) years, the disease duration was $99.1 \pm 72.6$ (range 6 to 324 ) months. The mean hospitalization period was 7.9 (range 4-14) days. Rate of hospitalizations for IIM was 0.79 patients/year, PGA- $43.9 \pm 24.0$ and MDGA-37.3 $\pm 21.8 \mathrm{~mm}$. The mean values SF-8 for physical compound was $36.5 \pm 9.0$ and for mental-41.7 \pm 9.6 , indicates reduced quality of life. The PSQ III results were: general satisfaction 18.8 \pm 2.9 , technical quality $34.7 \pm 5.3$, interpersonal aspects 24.9 \pm 4.5 , communication $18.4 \pm 3.7$, financial aspects $20.7 \pm 4.6$, time spent with doctor was $6.5 \pm 1.1$ and access/availability/convenience indicated $42.0 \pm 6.5$ score. We found a high negative correlation between both PGA and MDGA with SF-8 PCS ( $p<0.005)$ and a moderate between PGA and SF-8 MCS ( $p<0.005)$. The PSQ III domains/ $P G A$ gave negative correlation $(r=-0.23, r=-0.34, r=-0.1, r=-$ $0.21, r=-0.3, r=-0.1, r=-0.3, p<0.005)$, respectively. The MDGA with SF-8 MCS and the PSQ III domains showed a weak negative correlation $(r=-0.27, r=-0.1, r=-0.2, r=-0.1, r=-0.1, r=-0.2 r=-0.2 r=-$ $0.1 \quad p<0.005$ ) Conclusion: The disease activity in miopathies involves diminishing the quality of life and patient satisfaction.

Keywords: idiopathic inflammatory myopathies, correlations

91. Lung Transplant Proposal in a Patient with Chronic Obstructive Pulmonary Disease which Presents Combined Pulmonary Fibrosis and Emphysema, Complicated with Pulmonary Hypertension

Chelmambet D Altay',2, Professor Miron-Alexandru Bogdan ${ }^{1,2}$, Assistant Lecturer Dragos - Cosmin Zaharia ${ }^{1,2}$ "Carol Davila", University of Medicine and Pharmacy, Bucharest, Romania, 2"Marius Nasta" Pneumoftisiology Institute, Bucharest, Romania 
Background: Combined pulmonary fibrosis and emphysema (CPFE) is a rare clinical entity, characterized by exertion dyspnea, upperlobe emphysema, lower-lobe fibrosis and severely diminished diffusion lung capacity for carbon monoxide (DLCO). Underlying cause of CPFE is unknown, but high incidence is reported on male, heavy smokers. CPFE is frequently complicated by pulmonary hypertension $(\mathrm{PH})$, significantly worsening its prognosis. Most treatments are less effective, the only therapeutic choice which increases life expectancy, remains lung transplantation. Case presentation: A 58-years-old male, heavy-smoker untill 2015 (50 pack-year), with occupational exposure to respiratory toxicity (aircraft engines emissions) for 40 years, known with chronic obstructive pulmonary disease treated with bronchodilators and long-term oxygen-therapy, requests a consultation at Pneumophtisiology Institute "Marius Nasta", in December 2016, accusing aggravating exertion dyspnea. Physical examination showed pulmonary hyperinflation and chronic respiratory failure ( $\mathrm{SaO}_{2}$ at rest $92 \%$, at minimal effort $85 \%$ ). Thoracic (T-scan displays severe apical bullous emphysema and basal interstitial fibrosis. Pulmonary function tests reveal severe obstructive ventilatory dysfunction, pulmonary hyperinflation and severely reduced DLco with reduced transfer coefficient (KCo). Echocardiography showed dilatations of the right cavities, pulmonary artery and inferior vena cava, tricuspid regurgitation and a systolic pressure in pulmonary artery (SPAP) estimated at 68 $\mathrm{mmHg}$. The patient received therapy with dual-bronchodilators, and home oxygen-therapy. The clinical follow-up performed in October 2017, reveals aggravated exertion dyspnea. Physical examination and CT-scan have not changed since 2016; echocardiography showed aggravation of PH (SPAP $79 \mathrm{mmHg}$ ), mildly improved spirometry (FVC and FEV 1 from $54 \%$ and $34 \%$ in 2016 to $69 \%$ and $43 \%$ in 2017), but lowered DLCO and KCo from $23 \%$ and $39 \%$ to $19 \%$ and $34 \%$. Considering the poor prognosis of CPFE, the clinical picture of the patient with the worsening of the symptoms, the significantly reduced DLco and severe $\mathrm{PH}$ internationally classified in group III, which does not benefit of vasodilator treatment, the patient has indication for lungs transplantation. Conclusion: The clinical form of CPFE is a severe entity, more rapidly progressive than the separate emphysema and fibrosis diseases, which makes lungs transplantation a more frequent choice. The patient is undergoing evaluation for this difficult procedure which is discussed further.

Keywords: chronic obstructive pulmonary disease, combined pulmonary fibrosis and emphysema, pulmonary hypertension, lungs transplantation

\section{Metastatic Nephroblastoma in 6-Month-old Infant}

Bundovski Z. Kristijan', MD PhD Slavica KostadinovaKunovska $a^{2}$

IInstitute of Pathology, University Child Clinic, Faculty of Medicine, Ss. Cyril and Methodius University of Skopje, Institute of Pathology, Faculty of Medicine, UKIM

Background: Nephroblastoma, (Wilms tumor )-is the most common kidney tumor of childhood occurring at age of 3 or 4 , at the earliest. It is associated with genetic diseases and syndromes, but it can also occur sporadically. Case presentation: We present an autopsy case of a 6-month-old infant with diagnosed and treated Wilms tumor with metastatic deposits. Macroscopic analyzes showed tumor tissue in the right kidney with hemorrhagic and necrotic areas. There were metastatic deposits in left kidney, left lung, pericardium, liver, mesenterium, paratracheal and paraaortal lymph nodes, cerebellum, as well as hydrocephalus. The microscopic examination showed embryonal tumor composed of tubular structures incorporated in immature fibroblastic stroma, which clearly indicated for Wilms tumor. This was confirmed by specific immunohistochemical staining with antibody against WT1. Pathological findings correlated with all MRI diagnostics made during the previous multiple hospitalizations of the infant. The most striking was the great dissemination potential of this tumor and the deposit in the cerebellum, which to our knowledge had not been described in Literature. Having in mind that the prenatal screening discovered hydrocephalus due to $C$ section was performed a few days before the physiological term for delivery, as well as the early age of onset and rapid course of the disease, the cerebellar metastasis most probably occurred prenatally and caused intrauterine aqueductal stenosis and subsequent hydrocephalus. Conclusion: Nephroblastoma usually gives early clinical symptoms and with the modern technique for diagnoses and treatment has good outcome and survival. This case did not have any genetic disorder or family history for the diseases and its clinical presentation and pathological examinations were completely unspecific and different from what has already been known, which makes it extremely rare. We hope that this case report will contribute and increase the research of this topic.

Keywords: nephroblastoma, kidney, hydrocephalus, pathology, pediatrics

\section{Multiplex Genotyping of Oncogenic Viruses}

Blejusca N Laura Maria', Lecturer Ursu Ramona Gabriela, $\mathrm{MD}, \mathrm{PhD}{ }^{1}$

"Gr. T. Popa" University of Medicine and Pharmacy, Iassi, Romania

Background: Head and neck cancer (HNC), with an estimated global burden of approximately 700000 incident cases, is the sixth most common malignancy reported worldwide and has a high case fatality rate of 380000 deaths per year. It is now well demonstrated that HPV is involved in a subset of HNC (e.g., oropharyngeal cancer), and EBV is known to be associated with other lymphoproliferative diseases such as Hodgkin's lymphoma and nasopharyngeal carcinoma. Very few is known about a possible association between HNCS and other oncogenic viruses (Polyomaviruses and Herpesviruses). Case presentation: We present here the case of A.C., male, 67 years old, smoker, with clinical diagnosis of valeculla, epiglotis carcinoma. $3 \mathrm{~mm}$ of fresh tumor was transported in Eppendorf tubes with RNA later solution. Testing was performed at IARC (International Agency for Research on Cancer) by LUMINEX multiplex genotyping for 61 viral agents by using type-specific multiplex genotyping (TS-MPG) assays. Multiplex type-specific PCR used specific primers for the detection of 19 probable/high-risk alpha-HPV types, 2 low-risk alpha-HPV types, 25 genus-beta HPV types, 10 polyomaviruses and 5 herpesviruses. The assay also includes assessment of the $\beta$-globin gene, to evaluate the presence and quality of extracted DNA. The tumor was positive for MCV (Merkel cellpolyomavirus), EBV 1 (Epstein- Barr Virus), HHV6 (Herpes lymphotropic virus), HHV8 (Kaposi's sarcoma-associated herpesvirus). Conclusion: We detected an HPV negative case that is HPVV and HHV positive; in this case of HPV negative HNC case, other oncogenic viruses may be involved. Additional research is required for clarifying the natural history of these viruses in HNCS, as virus detection would have a decisive impact on diagnostic/decisional algorithms.

Keywords: HNC, oncogenic viruses, multiplex genotyping

\section{A Case Report of Silver-Russell Syndrome}

Capatina M. Elena-Maria', MD PhD Radulescu Adela ${ }^{2}$ "Carol Davila" University of Medicine and Pharmacy, Bucharest, Romania, 2"Alessandrescu-Rusescu" National Institute for Mother and Child Health, Bucharest, Romania

Background: Silver-Russel syndrome (SRS) is a rare condition characterised by intrauterine and postnatal growth retardation associated with characteristic features, such as relative macrocephaly, prominent forehead, body asymmetry and feeding difficulties. The purpose of this case report is to draw attention to this underdiagnosed condition, and the importance of early diagnosis, due to impending hypoglycemia, requiring constant monitoring and to present the management challenges. Case presentation: Female patient M.M., aged 5 months, presents with 
subfebrile state, diarrhea and dry cough. Due to her weight, simptoms and genetic syndrome, admission is decided. Born through cesarean section (pelvic presentation), at full term, with intrauterine growth retardation (birth weight $=1790 \mathrm{~g}$, length $=42$ $\mathrm{cm}, \mathrm{HC}=34 \mathrm{~cm}, \mathrm{TC}=25 \mathrm{~cm}$ ), Apgar score 6, born with an enlarged cranial diameter, diminished muscle tone, diminished motility, low-set ears, micrognathia, flat nose, protruding forehead, short sternum and arms, inferior limb asymmetry, fifth finger clinodactyly, labia majora not covering labia minora, clitoral hypertrophy. She was admitted in the Neonatal ICU and presented jaundice in the fifth day of life, treated with phototherapy ( 1 day). She is the second born in a family with no history of similar features. Physical assessment: weight $=3500 \mathrm{~g}$; height $=50 \mathrm{~cm}$; lower limb asymmetry (50/51 cm-right/left leg), distended abdomen, cardiac murmurs and genital abnormality. The rest of the physical examination was normal. The genetic consultation recommended: growth monitoring; orthopedic assessment; echocardiography; endocrinological evaluation; Diagnosed with enterocolitis and recovered uneventfully. Conclusion: The diagnosis of SRS is mostly clinical. In addition to the characteristics presented above, patients may also present with some of the following (by frequency): triangular face, fifth finger clinodactyly, shoulder dimples, micrognathia, low muscle mass, excessive sweating, low-set ears, down-turned mouth, high pitched voice, prominent heels, delayed closure of fontanelle, urogenital abnormalities, speech delay, irregular or crowded teeth, motor delay, syndactyly of toes, hypoglycemia, scoliosis and/or kyphosis. The patient discussed presents 12 out of these 18 features and is being monitored for associated management challenges. The conclusion of this case report is, first of all, that early diagnosis is essential in order to ensure proper development of the child, and that patients with SRS, even when presenting with minor symptoms, should be closely monitored, due to the risk of hypoglycemia.

Keywords: Silver-Russel syndrome (SRS), genetic disorders, imprinting disorders

95. Atrio-Ventricular Septal Defect Complicated with Severe Pulmonary Artery Hypertension in Down Syndrome

Florean N. Maria-Delia', Gonciar D Diana', Assistant Lecturer Militaru Mihai'

'Iuliu Hatieganu" University of Medicine and Pharmacy, ClujNapoca, Romania; Paediatric Hospital Cluj-Napoca, Romania

Background: Down Syndrome represents by far the most intense studied genetic disease, shedding light on the importance of chromosomial abnormalities in congenital heart defects, which are found in $40-50 \%$ of cases. Atrio-ventricular septal defects (AVSD) or atrio-ventricular canal defects are known to be found in up to $45 \%$ of the patients, leading to a significant mortality rate of $3,6 \%$. Case presentation: We report the case of a two months and three weeks old female infant, admitted to the Intensive Care Unit of Paediatric Hospital Cluj-Napoca with haematemesis, diarrhea, acute dehydration and anaemia. The patient was diagnosed with Down Syndrome at birth, after specific fenotype was observed and the karyotype showed nonmosaic trisomy 21. During hospitalization, ASTRUP parameters proved metabolic acidosis. Echocardiography emphasized AVSD and pulmonary artery hypertension (PAH). The patient was rehydrated, received medication for heart failure and antibiotics for bronchopneumonia. Few days later, the patient developed severe dyspnea, hyperpnea, progressive cyanosis and irritability, suggestive for hypoxic spell. After being placed in genupectoral position, nutritional rebalancing and midazolam administration, the general state improved, but the next day intense pallor was observed and ASTRUP indicated severe lactic acidosis. Echocardiography was used to assess the ejection rate of the right ventricle, which was less than $50 \%$, leading to the diagnosis of acute right ventricular failure. Following prostacycline administration, the patient became stable. Unfortunately, the prognosis remains poor because surgery for AVSD can not be performed in this case. Conclusion: Congenital heart disease is the major cause of morbidity and early mortality in patients with Down Syndrome. This case report presents the evolution of AVSD, complicated with severe PAH and heart failure and strengthens that rapid management is crucial for life expectancy improvement.

Keywords: Down Syndrome, pulmonary artery hypertension, hypoxic spell, atrio-ventricular septal defect

\section{Early Infantile Epileptic Encephalopathy Due to Mutation in KCNQ2 gene}

Rucka G Liene', MD Sandija Stanke2, MD Ilze Meldere ${ }^{1,2}$, MD Jurǵis Strautmanis ${ }^{1,3}$

'Riga Stradins University, Riga, Latvia, ${ }^{2}$ Children`s Clinical University Hospital, Neonatal Intensive Care Unit, Latvia, ${ }^{3}$ Children`s Clinical University Hospital, Neurology Department, Riga, Latvia

Background: Ohtahara syndrome (OS), or early infantile epileptic encephalopathy (EIEE), is usually diagnosed before the age of 3 months. Many seizure types can be seen in OS, though tonic seizures lasting seconds are seen most often. The diagnosis is based on clinical features and electroencephalography (EEG), where burst suppression pattern is seen. Possible causes may include brain malformations, metabolic disorders and certain gene mutations. One of the possible genes is $\mathrm{KCNQ}_{2}$ (potassium voltagegated channel subfamily $Q$ member 2 ), which encodes a protein that functions in the formation of the $M$ channel - a slowly activating and deactivating potassium channel that plays a critical role in the regulation of neuronal excitability. Treatment with sodium channel blockers is considered most effective. Case presentation: 11 day old male infant was brought to Emergency department with complaints of poor feeding, jaundice and seizures. First symptoms were seen on the 1st week of life, when the patient had temporary tonic seizures, with head turning and high pitched cry afterwards. EEG showed burst suppression pattern, which is seen in patients with OS. In magnetic resonance imaging no pathology was found. The patient continued to have focal onset seizures that were lasting for 10-30 seconds about 10 times per day. The seizures were resistant to phenobarbital, levetiracetam, vigabatrin. Ketogenic diet led to significant worsening. EIEE with unknown etiology was diagnosed, so tests for aminoacid analysis in plasma, urine and cerebrospinal fluid (CSF), and organic acid analysis in urine and CSF were made. The genetic testing showed a pathogenic heterozygous variation $\mathrm{C} .1678 \mathrm{C}>\mathrm{T}$ in the $\mathrm{KCNQ}_{2}$ gene, confirming EIEE. Seizures stopped with topiramate treatment at the age of 2 months, however EEC showed continuous multifocal epiletiform activity and neurological examination was suboptimal (limited eye tracking, axial hypotonia, limb hypertonia), therefore carbamazepine was prescribed at the age of 3 months. Further evaluation of the effect of the treatment will follow. Conclusion: In neonatal patients with early infantile epileptic encephalopathy attempts to find precise etiology should be made. Cenetic testing is recommended if structural and metabolic causes are excluded. Knowledge on specific genetic disorder can help in treatment decisions and possibly improved outcomes.

Keywords: Ohtahara syndrome, early infantile epileptic encephalopathy, seizures, burst suppression, KCNQ2

\section{Epileptogenic Effect of the Poisoning with Isonicotinylhydrazide}

Mariuta V. Magda-Alexandra', Ionescu D. TheodoraDaniela', Ivanciu 0. George Emilian 1, Constantin - Cosmin C Tărnăuceanu', Assistant Lecturer Irina Ciomaga1, MD PhD Catalina Maria Ionescu ${ }^{2}$

"Grigore T. Popa" University of Medicine and Pharmacy, laşi, Romania, "'Sf. Maria" Emergency Clinical Hospital for Children, Iaşi, Romania

Background: Isonicotinylhydrazide or Isonicotinic Acid Hydrazide is the most used medication for tuberculosis. The epidemiological 
impact of tuberculosis in Romania and the administration of Hydrazide in the family can orientate a difficult diagnosis with unresponsive afebrile seizures, to acute poisoning with Isonicotinic Acid Hydrazide. Vitamin B6 in its pyridoxine form is the antidote for this kind of poisoning that minimizes the risk of peripheral nerve damage. Case presentation: In the following, we will illustrate the case of a 16 year old girl found unconscious with generalized tonic-clonic seizures (grand-mal seizures). The seizures didn't respond to any type of anticonvulsants administered in the ambulance. The teenager lives with her boyfriend and has a 9 months old baby. The extended anamnesis revealed that she took a 6 months treatment with Isonicotinylhydrazide and she also came to the hospital two years ago because of poisoning with caustic substances. At the clinical exam the Glasgow Score was 4 , the arterial oxygen saturation was $94 \%$, she was tachycardic and she had miosis and trismus. In the absence of any other data about the patient combined with the unresponsive seizures, coma and lactic acidosis at a patient that had a background of family problems, doctorsput the question of poisoning, probably with Hydrazide. 5 grams of Vitamin B6 were administrated as an antidote to the poisoning with this antituberculosis drug and Sodium Bicarbonate for acidosis. The response was propitious, the seizures have stopped and the girl has regained consciousness. After that, the patient declared that she ingested 9 grams of Hydrazide (30 tablets) and alcohol (blood alcohol concentration - $136 \mathrm{mg} / \mathrm{dL}$ ). The patient was admitted to the toxicology department of the "Sf. Maria" Children Hospital for 5 days, in order to continue the treatment. She also took a psychological and psychiatrist evaluation. Conclusion: To conclude, Isonicotinylhydrazide is a highly neurotoxic drug that can lead to the alteration of the mental status, coma, seizures, hepatotoxicity, refractory metabolic acidosis and even death if it is not recognized and treated on time.

Keywords: Isonicotinylhydrazide, vitamin B6, poisoning, seizures, teenager

98. Evaluation of Platelet Indices in Overweight and Obese Children

Koç VK Cökhan', Balpetek IB Emre', Professor Orkide Donma $^{2}$, Professor Mustafa Metin Donma ${ }^{3}$

'Namık Kemal University, Tekirdağ, Turkey, 2Department of Biochemistry, Istanbul University, Cerrahpasa Medical Faculty, Istanbul, Turkey, ${ }^{3}$ Department of Pediatrics, Namik Kemal University Medical Faculty, Tekirdağ, Turkey

Background: Platelet indices are potentially useful markers for the early diagnosis of thromboembolic diseases. Obesity is a health problem also with effects on venous thromboembolism. This study has been designed to investigate the possible variations in platelet count (PLT), mean platelet volume (MPV) and platelet distribution width (PDW) in overweight as well as in obese state of children. Materials and methods: 74 obese (OB) and 28 overweight (OW) children aged 06-18 years were participated in the study. WHO diagnostic criteria based upon age and sex percentiles designed for obesity were used to classify $O B$ and $O W$ children. Weight, height, waist circumference, hip circumference, head circumference and neck circumference measurements were performed. Platelet count, platelet indices, MPV as well as PDW were determined using complete blood count analyzer. Statistical analyses were performed using SPSS17.0 statistical package. Results: No statistically significant difference was observed between mean ages (11.9 \pm 2.2 years vs $10.6 \pm 2.9$ years) of $O B$ and OW groups $(p=0.215)$. Body mass index values $(24.9 \pm 2.7 \mathrm{~kg} / \mathrm{m} 2$ in $O B$ vs $20.8 \pm 2.7 \mathrm{~kg} / \mathrm{m} 2$ in $O W$ chidren) were significantly differed $(p, 0.001)$. There was no statistically significant difference between waist-to-hip circumference ratios of the groups. However, head-toneck circumference ratio was significantly higher in ow group $(p=0.015)$. Platelet counts of $O B$ children were $326.24 \pm 68.34$ and of OW children were $345.56 \pm 87.75 \quad(p=0.133)$. Mean platelet volumes of the groups were almost the same $(8.6 \pm 0.9$ in $\mathrm{OB}$ and $8.5 \pm 0.7$ in
OW children) $(p=0.455)$. Platelet distribution width values for the groups were determined as $14.2 \pm 2.5$ and $10.8 \pm 5.5$ in $O B$ and $O W$ children, respectively $(p=0.017)$. Conclusion: Platelet activation is thought to be involved in venous thromboembolism. MPV and PDW are simple platelet indices, which increase during platelet activation. Only PDW showed a significant increase transmitted from overweight state to obesity state during childhood. PDW is found to be a more specific marker of platelet activation, since it does not increase during simple platelet swelling.

Keywords: Platelet indices; thromboembolism; childhood obesity

\section{Kawasaki Disease in a 4-Months-Old Girl}

Vlaicu M Tudor', Lupu Aurica', Balan Ioana-Georgiana', Tomi Iulia', Professor Horvath M. Adrienne², Assistant Lecturer Voicu Sanda Luciaz

'University of Medicine and Pharmacy, Tirgu Mures, Romania, zpaediatric Clinic II Tîrgu Mures, Romania

Background: Kawasaki disease (KD) is a vasculitis occurring in infants and children characterized by prolonged fever polymorphous skin rash; erythema of the oral mucosa, lips and tongue; desquamation and swelling of the palms and soles, bilateral conjunctival injection. KD is the most common cause of multisystem vasculitis in childhood; the most commonly damaged are the coronary arteries. Case presentation: A 4-months-old girl previously well was admitted to the Odorheiul Secuiesc hospital with a history of fever for 2 days $(39,90 \mathrm{C})$; at home was administrated 2 doses of amoxicillin. The treatment was stopped because of erythematosus exanthema on the face and neck. Laboratory results on admission showed an elevation of inflammatory indices: WBC:23000/mcL with $58,6 \%$ neutrophil, CPR:108mg/L. It was interpreted like bacterial infection with unknown focus of infection and were given second and thirdgeneration cephalosporins. There were performed pulmonary RTG, cranial $C T$, bacterial culture from ear secretion, Ab anti Epstein Barr, Parvovirus B19, Measles virus, stool culture and all were negative for infection, but fever persisted. The patient is transferred to Paediatric Clinic II Tirgu Mures. There was performed an abdominal echography which related the left kidney $1 \mathrm{~cm}$ bigger than the right one, left pyelectasis $(12 / 14 \mathrm{~mm})$ and calyx distended (6mm); laboratory: WBC:37000/mcL, neu:24600/mcL, CPR:28,3, urine culture -negative. The results were interpreted like pyelonephritis and treatment was continued with: cefuroxime, amikacin. On the second day, the fever decreased, but reappeared shortly after $(39,80 \mathrm{C})$. Retaking the anamnesis, mother remembered that the patient had rash, conjunctivitis, cracked lips, erythema of fingers. It was decided to perform a cardiac echography which discovered: dilated right and left coronary artery ostium and ADA. The diagnosis was Kawasaki disease and the patient received i.v. immunoglobulins. Conclusion: After multiple investigations and treatment without any result, the persistent fever with rash, conjunctivitis, cracked lips, erythema of fingers which appeared gradually and cardiac echography put the diagnosis of Kawasaki disease, despite of pyelonephritis that delayed it. A successful response was finally obtained: remission of the patient's clinical symptoms within one week, associated with a gradual decrease in the diameters of the aneurysms.

Keywords: Kawasaki disease, pyelonephritis, coronary artery dilatation

\section{Lissencephaly - A Smooth Brain and a Rough Journey}

Zaharia G Marius-Cristian, Botez C Andreea-Cristina, MD Diana Epure

"Carol Davila" University of Medicine and Pharmacy, Bucharest, Romania

Background: One of the most striking abnormalities of human neuronal migration is lissencephaly (or "smooth brain"). The two most common genes mutated in patients with lissencephaly are 
LIS1 and DCX. There are two major types of classical lissencephaly: isolated lissencephaly sequence (ILS) and Miller-Dieker syndrome (MDS). Miller-Dieker syndrome (MDS) consists of more severe lissencephaly than ILS patients, characteristic facial anomalies (high forehead, a small nose with anteverted nares, thin vermilion border, and micrognathia), and occasionally other malformations. Children with ILS and MDS are severely retarded and suffer from epilepsy. Case presentation: We present the case of a 14-year-old patient known for lissencephaly. Although she showed signs of mental retardation at 3 months, the first seizures occurred 3 months later, at 6 months, after the administration of the antipolio vaccine, the patient developed focal motor seizures. Several treatment plans have been tried, best responding to prednison and tetracosactide hexaacetate. Imaging investigations, conducted at 6 months, lead to the diagnosis of lissencephaly. The presence of facial dysmorphism, epilepsy and psychomotor retardation leaded to suspicion of Miller-Dieker syndrome. Also, her hands and her legs are small in relation to the length of the limbs and presents an important thoracolombar scoliosis. Conclusion: Lissencephaly is a rare congenital malformation of the brain that has clinical and radiographic characteristics. Patients have severe mental retardation and seizures. Life expectancy is related to the severity of the lissencephaly. Despite the fact that many children with Miller-Dieker syndrome (MDS) do not live past age 2, and only a few may reach age 10, our patient has managed to survive up to 14 years already.

Keywords: Lissencephaly, dysmorphism, seizures, Miller-Dieker syndrome

101. Missing Clues to Diagnosis: Hypomelanosis of Ito in a 10Month-Old Infant

Buțurcă M Maria Diana, Ilie T Maria-Teodora, Delia - Irina

L. Vasile, Cosoreanu G Vlad, MD Daniela Stoian

"Carol Davila" University of Medicine and Pharmacy, Bucharest, Romania

Background: Hypomelanosis of Ito is a rare congenital disorder characterized by hypochromic skin lesions presenting as patches and whirles along the lines of Blaschko. Approximately $75 \%$ of the patients associate systemic nervous and musculoskeletal manifestations. A precise cause remains unknown, although an abnormal karyotype is often present(chromosomal mosaicism, $X$ chromosome activation/inactivation). Prognosis is heterogeneous, being determined by the severity of the coexisting conditions, but death rarely occurs. Case presentation: In 2010, a 10-month-old male patient presents for delayed development, hypopigmented lesions and hemihypertrophy of the right side of the body. Medical history includes an Apgar score of 8 and an older brother with congenital hypoplastic kidney and hydronephrosis. At 14 months, when first generalized tonic clonic seizures occur, treatment is proposed, but the family shows low compliance. At the age of 2, MRI and karyotyping are performed and the results are normal. The frequency of seizures is 3-4/year, until 2013, when it increases and treatment with Valproic acid is initiated, but no control is obtained. In spite of introducing Levetiracetam and Clobazam one and two years later, respectively, the episodes reach a maximum of $5 /$ day. Meanwhile, the association of global developmental delay, seizures, macrocrania, hypotonia, hemihyperthrophy and hypopigmented lesions lead to a late diagnosis of Hypomelanosis of Ito. Because of an increase in seizure frequency, low doses of dexametazone are administered, but the patient develops acute liver failure and dies in the intensive care unit. Conclusion: Considering the rarity of this neurocutaneous disease and the lack of proper tests, diagnosis is difficult to be made, but reaching it is important in order to rule out other treatable maladies. The main problem in managing this case was the absence of response to all the antiepileptic drugs that were used. Dramatic progression of the neurological manifestations and premature death are significant particularities, as this course of events is uncommon in hypomelanosis of Ito.
Keywords: Hypomelanosis of Ito, tonic clonic seizures, developmental delay, hypochromic skin lesions

102. Neurological Damage and the Importance of Newborn Screening in PKU

Apostol N Alina Georgiana', MD PhD Assistant Ana-Simona Drochioi², Lecturer Dana-Teodora Anton Paduraru

"Grigore T. Popa" University of Medicine and Pharmacy, lasi, Romania, z"Sf. Maria" Emergency Clinical Hospital for Children, Iassi, Romania

Background: Phenylketonuria (PKU) is an autosomal recessive inborn error of metabolism caused by a deficiency of the enzyme phenylalanine hydroxylase (PAH) which catalyses the conversion of the amino acid phenylalanine (Phe) into tyrosine. The enzyme deficiency results in a high concentration of Phe, which is neurotoxic particularly during the first years of life. The damage done to the brain if PKU is untreated during the first months of life is not reversible. Clinically, the baby with PKU looks normal at birth, but in the following 3 to 4 months the signs start to show. The most severe symptoms are the intellectual disabilities and the neurological problems that include generalized hypotonia or hypertonia, spastic paraplegia, exaggerated deep tendon reflexes. The patients' sweat and urine odor is mousy or musty. The one year old child presents hyperactivity, spontaneous movements, athetosis. Without treatment, mental retardation is imminent: only $5 \%$ of the patients have an IQ over 68 . Materials and methods: The study lot consisted of 17 patients, aging between 28 days and 13 years old, hospitalized in the 3rd Pediatrics Clinic at "Sfanta Maria" Hospital, laşi between 01.01.2011 - 01.11.2016 and diagnosed with phenylketonuria. The study was based on analyzing the patients' observation files. Results: Most of the patients were diagnosed by screening immediately after birth and their evolution is favorable with the specific diet, but one of them has not benefited of screening, being diagnosed late and currently has severe neurological impairment. Conclusion: Early diagnosis of the patients with phenylketonuria leads to creating a nutritional treatment which can avoid severe further neurological complications if strictly followed.

Keywords: phenylketonuria, phenylalanine, newborn screening, neurological damage, diet

103. Occurrence of Acute Kidney Injury in Term Neonates with Severe Perinatal Pathology: Role of Therapeutic Procedures Isa Mashkur' ${ }^{1}$, Associate Professor Babintseva Anastasia ${ }^{2}$ 'Bukovinian State Medical University, Cernăuţi, Ukraine, ${ }^{2}$ Department of Pediatrics, Neonatology and Perinatal Medicine, Bukovinian State Medical University, Cernăuţi, Ukraine

Background: Term newborns with perinatal pathologies (asphyxia, hypoxic encephalopathy or hemolytic disease) have the kidneys as the most damaged organ. Hence, they are prime candidates for the development of acute kidney injury (AKI). With AKI affecting about $30 \%$ of hospitalized neonates, we aim to determine the association of therapeutic procedures with its occurrence. Materials and methods: A comprehensive clinical-paraclinical examination of 95 term newborns with clinical signs of severe perinatal pathology was carried out; 65 of them having disorders of renal function, and 30 - with AKI. The connection between the character of therapeutic procedures and development of AKI was investigated using a logistic regression analysis by calculating the chance correlation (CC) and $95 \%$ confidence interval $(95 \% \mathrm{CI})$. Results: Postnatal factors increasing the risk of AKI formation and aggravating renal dysfunction in critically ill term babies have been found to be: the use of oxygen therapy with free flow (CC 3,13; $95 \% \mathrm{Cl} 1,059-9,225, \mathrm{p}<0,05)$, loop diuretics (CC 15,8; $95 \% \mathrm{Cl}$ 4,035-61,901, p<0,05), medications with inotropic effect (CC 9,0; $95 \% \mathrm{Cl} 3,187-25,41, \mathrm{p}<0,05)$, antispasmodic drugs (CC 17,38; $95 \% \mathrm{Cl}$ $3,818-79,117$, p<0,05), fresh frozen plasma (CC 5,14;95\% Cl 1,548$17,09)$ against a background of combined antibiotic therapy and application of ventilation support. Conclusion: The use of therapeutic procedures aggravates unfavourable effects of hypoxic 
renal lesion and increases the risk of AKI formation in term newborns with severe perinatal pathology, which is associated with oxidative stress, disorders of central and peripheral hemodynamic mechanisms, direct cellular lesions of the glomerular membrane and canalicular nephrothelium.

Keywords: acute kidney injury, term newborns, mechanical lung ventilation, oxygen therapy, inotrops

104. Prader Willi Syndrome - Diagnosis in an Infant Dobre V Alexandra Marinela ${ }^{1,2}$, MD Apostol Irina

"Carol Davila" University of Medicine and Pharmacy, Bucharest, Romania, ${ }^{2 " A l f r e d ~ R u s e s c u " ~ N a t i o n a l ~ I n s t i t u t e ~ f o r ~ M o t h e r ~ a n d ~ C h i l d ~}$ Health, Bucharest, Romania

Background: Prader-Willi syndrome (PWS) is a rare genetic condition that causes a wide range of problems: floppiness and weak muscles, feeding problems and failure to grow during the first year of life, rapid weight gain in children aged one to six years, delayed physical development or learning difficulties. It is caused by a defect on the paternal chromosome number 15 , which happens purely by chance. Case presentation: 1 month and 10 days infant transferred from a regional hospital for further investigations and multidisciplinary consults concerning a congenital hypotonic syndrome. He had, among other things, generalised hypotonia, cryptorchidism, congenital stridor, oedemata. His state indicates a genetic disorder, his lack of growth and craniofacial dysmorphism lead to a few differential diagnosis. During his multiple stays at the hospital, he has been tested for Zellweger syndrome, spinal amyotrophy, Pompe disease and Prader-Willi syndrome. Because of his poor alimentation, a gastrostomy was needed. The therapeutic approach was about improving his weight and curing various infections associated with the long period of time spent in the hospital. Conclusion: After multiple in hospital stays and tests the diagnosis of Prader-Willi syndrome was confirmed, indicating the difficulty of recognizing this malady in young children because it does not take the same form as it does in older patients. The treatment is only palliative and it's about making the lives of patients living with this disease as normal as it can be.

Keywords: Prader-Willi Syndrome, genetic disorder, hypotonia, failure of growth

105. Red Blood Cell Distribution Width During 0verweight Stage Towards Childhood Obesity

Balpetek IB Emre', Koç VK Gökhan ${ }^{1}$, Professor Orkide Donma ${ }^{2}$, Professor Mustafa Metin Donma

'Namık Kemal University, Tekirdağ, Turkey, ${ }^{2}$ Department of Biochemistry, Istanbul University, Cerrahpasa Medical Faculty, Istanbul, Turkey, ${ }^{3}$ Department of Pediatrics, Namik Kemal University Medical Faculty, Tekirdağ, Turkey

Background: Red blood cell distribution width (RDW) is a simple parameter traditionally used in laboratory hematology for differential diagnosis of anemia and its potential is recently being noticed for multiple clinical applications as in coronary artery disease. In this study, the possible use of this parameter in the obesity development in children was investigated. Materials and methods: A total of one hundred and ten children (aged 06-18 years) were included into the scope of this study. Twenty eight of them were overweight (OW) and 82 were normal weight (NW). Their weights and heights were measured and body mass index (BMI) values were calculated. Anthropometric measurements were performed. WHO-BMI values of age-and-sex-percentile curves designed for 5-19 year-aged-children were used for determining OW and NW criteria. RDW-CV was determined using hematology analyzer. Statistical analyses were performed using SPSS17.0 statistical package program. Results: Mean ages were 10.6 \pm 2.9 years for OW and $9.2 \pm 2.1$ years for NW groups. There was no statistically significant difference between ages of the groups. BMI values were $20.8 \pm 2.7 \mathrm{~kg} / \mathrm{m} 2$ and $15.8 \pm 1.1 \mathrm{~kg} / \mathrm{m} 2$ for $\mathrm{OW}$ and NW groups, respectively. A statistically significant difference was noted between BMI values $(p, 0.001)$. Waist-to-hip $(p=0.526)$ and head-to-neck ratios $(p=0.702)$ did not statistically differed between groups. Red blood cell counts (RBC) of groups were almost the same. RDW-CV differed significantly between OW (14.97 \pm 5.77$)$ and NW $(13.78 \pm 1.06)$ children $(p=0.030)$. Conclusion: Obesity is gradually increasing health problem. Childhood obesity gains importance for the prevention of this problem in advance. RDW is a measure of RBC volume variations. RDW-CV is found to be elevated in $\mathrm{OW}$ children. This finding becomes even more important in the presence of similar C-reactive protein, insulin resistance index, leukocyte and platelet count as well as indices. This may be the predictor of impaired blood flow through microcirculation, resulting in decreased tissue oxygen availability, which participates in low-grade inflammation in obesity.

Keywords: Red blood cell distribution width, coronary artery disease, childhood obesity

106. A 10 Hour Surgery Against a Periorbital Recurrent Squamous Cell Carcinoma

Negrila F Alina - Alexandra', MD Manuel Loo Olivares $^{2}$ 'University of Medicine and Pharmacy of Craiova, Craiova, Romania, 2Regional Hospital of Antofagasta, Antofagasta, Chile

Background: The second most common form of skin cancer, squamous cell carcinoma, is mainly caused by cumulative ultraviolet exposure and affects skin's upper layers. Anyone who has had one squamous cell tumor has an increased chance of developing another, especially in the same skin area or nearby. Left untreated, they eventually penetrate the underlying tissues and can become disfiguring. Removal of a larger tumor often requires reconstructive surgery, involving a skin graft or flap to cover the defect. When it spreads, squamous cell carcinomas frequently can be life-threatening. In certain geographic locations, exposure to arsenic in well water or from industrial sources may significantly increase the risk of this disease. Case presentation: A 72-year old female with a history of skin cancer multioperated at the left cheek and treated with volumetric modulated arc radiotherapy presents a new lesion in the outer canthus of the eye with neoplazic edema involving lower eyelid, eyebrow and cheek. It invades the orbit and the intraconal region with displacement of the eyeball towards superior and lateral. Inferior rectus muscle is also suspected of infiltration. The surgery involves an incision with a margin of $1 \mathrm{~cm}$ of the compromised areas including eyeball, bone of the orbit, zygomatic and maxillary, leaving a large defect communicating with nasal fossa and high cervical region. It is required reconstructive surgery with a free flap of $15 \mathrm{~cm}$ diameter from the rectus abdominis muscle which is sutured to facial vessels by micro-surgery. Conclusion: A squamous cell carcinoma can recur even when it has been carefully removed the first time. While squamous cell carcinomas and other skin cancers are almost always curable when detected and treated early, it is best to prevent them in the first place. Anyone with a history of substantial sun exposure is at increased risk. The cancer can arise in burns, scars, ulcers, long-standing sores, and sites previously exposed to X-rays or certain chemicals (such as arsenic and petroleum by-products). Early diagnostic avoids complications for this disease. Still,the incidence of squamous cell carcinoma continues to rise around the world. It represents an important field of research which needs constant awareness.

Keywords: skin cancer, squamous cell carcinoma, sun exposure, arsenic exposure, plastic surgery

\section{A Personal Approach in the Surgical Treatment of Rhinophyma}

Petrea D.D Cosmin Mihai', Vintila M Cristian', Gheorghe Flavian I Tutuianu', Gabriela L Madarasz', MD PhD Adrian Botan', Associate Professor Dan Viorel Cosma' University of Medicine and Pharmacy, Tirgu Mures, Romania 
Background: Rhinophyma is a hypertrophic proliferation of the sebaceous glands of the lower half of the nose (a tumoral form of rosacea), associated with increased fibrosis and vascularity and according to the 0xford Medical dictionary, rhinophyma is in no way related to alcohol abuse. The disease is more prevalent in men (men/women ratio:10/1) and has sometimes a family history. Case presentation: A 73 years old patient was appointed to a surgical treatment involving the removal of a giant rhinophyma developing on the lower half of the nose. The hypertrophic process was obstructing the external nasal valves, which was leading to a secondary nasal airway obstruction suffering. Under general anesthesia it was performed a complete excision of the entire esthetic nasal unit, removing all tissues involved in the hypertrophic process, but leaving a thin layer (about 2-3mm) above the nasal perichondrium. The secondary defect was covered by a full-thickness-skin-graft (FTSG), harvested from the lower abdomen; the graft was secured with long string sutures in order to place a bolster dressing. Two months later we can see the final results with an acceptable nasal shape and a slight hyperpigmentation of the graft which will disappear in the next months. Conclusion: This procedure is a very safe way to deal especially with giant rhinophyma requiring large excision and meticulous haemostasis. The full-thickness-skin-graft had a very good evolution with no complication and good aesthetic results. In addition, removing the entire nasal pathologic tissue, there was no possibility of a relapse.

Keywords: rhinophyma, skin graft, hypertrophic process

108. A Rare Case of a Sellar and Suprasellar Immature Teratoma Axinia V Iulia- Georgiana, Ilășoaia F Amalia - Elena, Duca F.N. Ștefania-Teodora, Anton E Sorana, MD PhD Bogdan Iliescu

"Gr. T. Popa" University of Medicine and Pharmacy, Iași, Romania

Background: The teratoma is a complex neoplasm which contains a variety of heterogeneous tissues, typically reflecting more than one of the three embryonic germ layers. The immature teratoma consists of less-differentiated elements derived from one or all germinal layers and may exhibit malignant behavior. The teratoma of the sellar region is rare and usually occurs in children and young adults. Treatments of resection, radiation therapy and chemotherapy are generally recommended for the control of aggressive immature teratomas. Case presentation: We are reporting the case of a 7 year old female patient, who was transferred to the Neurosurgery Department for a sellar and suprasellar tumor revealed by the imagistic explorations. Prior to the transfer, the possibility of an infectious pathology was excluded. At the MRI investigation there was shown a tumoral mass of approximately $34 / 29 \mathrm{~mm}$, which seemed well defined, with a non-homogenous structure, cystic formations and high signal intensity on T1 and T2 weighted MRI images. The suspicions made after the imagistic findings included adenoma, craniopharyngioma and optic chiasm tumor. The patient also presented intracranial hypertension syndrome, polyuria-polydipsia syndrome, gelastic seizures, bilateral amblyopia, hypothalamopituitary disconnection and adrenocorticotropic hormone deficiency. The surgical treatment consisted in the subtotal ablation of the tumor using the CUSA system (Cavitron Ultrasonic Surgical Aspirator) subsequent to an interhemispheric transcallosal approach. The intraoperative aspect of the tumor resembled a germinoma, but the anatomopathological report revealed the diagnosis of immature teratoma. The surgery had a positive outcome. Conclusion: The particularities of this case consist in the location of the tumor and its connection with different anatomical structures, as well as the imagistic and histological aspects which made the confirmed diagnosis a true challenge.

Keywords: immature teratoma, suprasellar tumor, CUSA, germinoma
109. A Real Challenge in the Management of the Postexcisional Defects for Giant Nodular Carcinoma of the Periocular Region

Para A Adina Georgiana'1,2, Tofan C Remus-Andrei',3, DanielGeorge C Boicu', Lecturer Mihaela Pertea',3

"Gr. T. Popa" University of Medicine and Pharmacy, Iași, Romania, ${ }^{2}$ Saint Spiridon Emergency Clinical Hospital, Iași, Romania, ${ }^{3}$ Clinic of Plastic Surgery and Reconstructive Microsurgery, Emergency University Hospital "St. Spiridon", Iași, Romania

Background: The giant basal cell carcinoma is a rare skin malignity, representing only $1 \%$ of the basal cell carcinomas. The giant type is defined as the lesion which exceeds $5 \mathrm{~cm}$ in diameter. The disease is reported, in general, at the persons in their seventh decade of life, patients with various other pathologies. The excision between oncological limits lead to large soft tissue defects which, if localized at the periocular region, are a real challenge for the surgeon that has to choose a surgical technique for the reconstruction. Materials and methods: The study includes 8 patients, 7 male and one woman, age between 60 to 85 years, with a history of carcinomatous lesions in evolution from 7 to 12 years. All the lesions have dimensions between 5 and $7,5 \mathrm{~cm}$, located in four cases in the external angle of the right eye, three at the upper eyelid and the external angle of the left eye, and, in one case, in the glabellar region with extension at both eyes. In all of the 8 cases the intervention consisted in complete excision (with oncological limit restriction) and covering with regional flaps (in 3 cases Mustarde flap, in 2 cases association of frontal flaps and in 3 cases genian advancement flap, from witch, one anchored in the zygomatic bone). All the reconstructive surgical interventions were performed in one operatory time, only in two cases it was neccesary the reintervention after three months for the sectioning of the conjunctival flap (for the eye protection). In all 8 cases the nodular form of the basal cell carcinoma was observed. Results: The immediate postoperative evolution was good, without flap vascularisation problems. Long term evolution was good, with full reintegration of the flaps and a pleasant esthetic result. No recurrences ware registrated 18 months after the intervention. Conclusion: The giant basal cell carcinoma, a rare form of disease, is most often diagnosed at advanced ages. On the face, excision determines the presence of large soft tissue defect. Sometimes the reconstruction represented a real challenge for the surgeon.

Keywords: carcinoma, flap, soft tissue defect

110. Aggressive Form of Pancreatic Ductal Adenocarcinoma Requiring Multiple Organ Resections.

Necula 0 Andra Carmina', lacob V. Stefan ${ }^{1,2}$, MD PhD Dr. Vlad Porumb'

"Gr. T. Popa" University of Medicine and Pharmacy, Iassi, Romania, ${ }^{2}$ The Regional Oncology Institute, Iași, Romania

Background: Pancreatic ductal adenocarcinoma (PDAC) is the most common malignancy of the pancreas. PDAC is an epithelial tumor that arises from the cells of the pancreatic duct and it is an aggressive and difficult to treat malignancy. According to the American Pancreatic Association's articles, the development of PDAC occurs over an extended period of time, and likely follows a stepwise progression similar to other carcinomas. This progression is characterized by the transition of the normal pancreatic duct to a pre-invasive precursor lesion known as pancreatic intraepithelial neoplasia, which can develop into an invasive PDAC. Case presentation: We admitted a 60-years old male patient in the oncology surgery unit in 2013, with important dysphagia, with the suspicion of a Gastrointestinal Stromal Tumor (GIST) which we detected during a superior endoscopy exam. During the surgery, a mass between the stomach and the colon was found for which we decided to perform gastrectomy and segmental colectomy, followed by chemotherapy treatment. After the surgery, the anatomopathological examination highlighted a well-differentiated ductal pancreatic carcinoma, which was present on a possible 
ectopic pancreas and, despite the chemotherapy treatment, we readmitted the patient multiple times in the surgery unit in 2016 for metastases. We executed right hemicoletomy, ileocolostomy, splenectomy, caudal pancreatectomy, left hemicolectomy, left nephrectomy and jejunal enterectomy with favourable postoperative evolution. In August 2016 we admitted the patient in order to perform a biopsy from a right inguinal mass, which came back positive for an adenocarcinoma characterized as having glandular structure. We excised the mass, along with the spermatic cord, in addition to right orchiectomy and right iliac venoraphy. In 2017 the patient underwent a CT-scan of the thorax, abdomen and pelvis which revealed the presence of iodophilic solid masses, with tendency to confluence, in the anterior abdominal wall. Following this result, we excised the periumbilical mass and performed a jejunostomy, with favourable evolution. Conclusion: The postoperative evolution from multiorgan resections was prolonged,but favourable despite the fact that the patient was suffering from an aggressive form of PDAC and that the surgical intervention was aggressive as well.

Keywords: surgery, pancreatic duodenal adenocarcinoma, multiorgan resection

111. An Elderly Woman with Triple Primary Metachronous Malignancy

Loghinoaia M Luciana-Maria', Constantinescu | Angela', Țugui A Denisa-oana', Tătaru C Radu-Cristian', Professor Scripcariu Viorel' ${ }^{2}$, Lecturer Radu Iulian²

"Gr. T. Popa" University of Medicine and Pharmacy, Iași, Romania, ${ }^{2}$ The Regional Oncology Institute, Iași, Romania

Background: Carcinoma of the ampulla of Vater is a malignant tumor arising in the last centimeter of the common bile duct, where it passes through the wall of the duodenum and ampullary papilla. Adenocarcinoma of the ampulla of Vater is relatively uncommon, accounting for approximately $0.2 \%$ of gastrointestinal tract malignancies and approximately $7 \%$ of all periampullary carcinomas. Case presentation: We present the case of S.0., a 68 year old female,with important pathological antecedents(malignant neoplasm of right breast-2013 and malignant neoplasm of rectosigmoid junction-2014), who was admitted to the Regional Oncology Institute in Iaşi accusing the following symptoms : right upper quadrant pain, severe nausea and vomiting. The paraclinical investigations (an abdominal Magnetic Resonance Imaging) raised the suspicion of a tumoral mass located on Vater's Ampulla. At admission the following were present: anemia and hepatocytolytic syndrome. The upper digestive endoscopy examination detected a duodenal bulb that causes extrinsic compression and an expansive bleeding mass at the level of the Ampula of Vater. After the preoperative measures have been taken care of, the surgery commences and a tumoral mass is found at the level of the head of the pancreas.The next step is to perform a cephalic duodenumpancreatomy. The histological exam confirms the diagnosis of adenocarcinoma: moderately differentiated ampullary type intestinal adenocarcinoma. The neoplasic tissue extends to the mucosa of the duodenum (the 2nd segment) and it infiltrates the muscular layer of the duodenum in all its thickness, penetrating the adjacent pancreatic tissue, but sparing the main pancreatic and common bile ducts. Conclusion: Multiple primary malignancy (MPM) is defined as occurrence of two or more malignancies in the same individual without any relationship between the tumors either simultaneously or in an interval of time. Although rare, triple tumors are seen in $0.5 \%$ of cases. An individual may develop MPM in a lifetime due to genetic predisposition, environmental exposure to carcinogens, immunodeficiency or as a serious complication of chemotherapy or radiotherapy received for the first primary malignancy. Finally, the patient had a favorable evolution being surgically healed.

Keywords: ampulla of Vater, malignant, metachronous

\section{Aorto-Right Atrial Tunnel: A Rare Pathology with Severe} Consequence

Slobozeanu S.P. Andrei', MD Alexandru Vasilescu², MD Andrei Danet ${ }^{2}$, MD Catalin Constantin Badiu ${ }^{2}$

'Carol Davila' University of Medicine and Pharmacy, Bucharest, Romania, 2Emergency University Hospital, Bucharest, Romania

Background: Aorto-right atrial tunnels (ARAT) are rare extracardiac or intracardiac vascular structures deriving from the aortic sinus, passing the aorta anterior or posterior and flowing into the right atrium (RA). We report two cases, along with their diagnostic findings and surgical treatment. Case presentation: A 21-year-old asymptomatic man presented with a 2 centimeters fistulous tract originating from the left coronary sinus, running downwards, being attached intracavitary to the left atrium, and draining into the RA cranially to an ostium secundum defect. Intraoperative, the tunnel was resected from the sinus, which was reconstructed with a Dacron patch. The left coronary artery was widely mobilized and implanted into the patch. Both ends of the ARAT were closed. The second case describes an anterior 1.5 centimeters tortous vascular structure, in a 27-year-old asymptomatic female, arising from the right coronary sinus, running posterior to the RA and opening laterally into it. Intraoperative, the tunnel was resected and closed at both ends, and the sinus was reconstructed with a Dacron patch. The origin of the right coronary artery could not be mobilized enough in order to avoid tension and thus, an end-toend anastomosis with the right mammary artery had to be performed. The postoperative recovery was uneventful in both cases. At six months follow-up imaging revealed a normal ventricular function, a normal aortic root shape without aortic insufficiency, and no residual shunt in both cases. Conclusion: The need for ARAT closure should be discussed controversial in asymptomatic patients, due to the complex, unstandardized surgical methods. Nevertheless, the risk of an increased left-toright shunt, may only postpone but not dispose operation.

Keywords: cardiology, surgery, aorto-atrial tunnel, pathology, rare, coronary sinus, atrium, ostium secundum, Dacron patch, coronary artery, anastomosis, mammary artery, asymptomatic

113. Autologous Breast Reconstruction with Deep Inferior Epigastric Perforator Artery Flap - Best Option for the Irradiated Field

Dobreanu M.A. Stefan Dorin', Tarcau S Bogdan- Marian', Otilia Andreea L Petcuţa', MD Codrin Nicolae Dobreanu²

'Gr. T. Popa" University of Medicine and Pharmacy, Iași, Romania, ${ }^{2}$ Oncology Institute of Iași, Romania

Background: Breast cancer is the leading cause of cancer death in female patients worldwide. Although the incidence is increasing every year, the improvement of the surgical techniques, adjuvant therapy and overall patient care has led to a decrease in breast cancer mortality. Thus, the reconstructive need increased, autologous tissue reconstruction also gaining ground over the more popular expander/implant technique. This trend can be observed even in Romania, where, due to gains in surgical expertise, the procedure is offered in a few centers. Case presentation: We present the case of a 51-year-old female, diagnosed with invasive NST right breast cancer for which she underwent breast-conserving surgery, resulting positive margins. Due to that, a radical modified mastectomy was performed in June 2015 , followed by adjuvant radiotherapy. In April 2017, the patient demanded a right breast reconstruction with autologous tissue, after being presented with the reconstructive options. A deep inferior epigastric perforator (DIEP) artery flap was harvested from the patient's lower abdomen and inset in the post mastectomy defect with microscope-assisted-anastomosis of the deep inferior epigastric pedicle to the right internal mammary artery and vein. The postoperative evaluations of the flap proved a good perfusion and having no other complications, the patient was discharged after 7 days. Conclusion: For a young patient with a history of 
radiotherapy, an autologous tissue flap reconstruction manages best the reconstructive needs in terms of volumes, aesthetics and number of surgical procedures needed to obtain the final result, compared to the expander/implant technique which requires regular revisions to obtain shape and symmetry. Furthermore, this patient benefited from associated abdominoplasty which was performed in order to close the donor site.

Keywords: breast reconstruction, autologous flap, DIEP flap, breast cancer

114. Belated Gastric Fistula Post-Sleeve-Gastrectomy Profire M Bianca-Stefania, Confederat Luminita, Matei Theodor, Minea T Horia-Octav, Assistant Lecturer Cristian Velicescu I"Gr. T. Popa" University of Medicine and Pharmacy, Iași, Romania

Background: Longitudinal sleeve-gastrectomy is a surgical procedure which is included in the restrictive category of bariatric surgery. This surgery is recommended for patients suffering from morbid obesity (BMI. $40 \mathrm{~kg} / \mathrm{m}^{\wedge} 2$ ) or in the case of comorbidities associated with a BMI. $35 \mathrm{~kg} / \mathrm{m}^{\wedge} 2$. Case presentation: A 37 -year-old female, who had undergone a sleeve-gastrectomy in Turkey (Istambul) in January 2017 for morbid obesity (200kg and $\mathrm{BMl}=55 \mathrm{~kg} / \mathrm{m}^{\wedge} 2$ ), arrived after six months in the Emergency Department of "Sfântul Spiridon" Emergency Clinical Hospital in cardiopulmonary arrest. After 20 minutes a resuscitation response was obtained and a thoraco-abdominal computed-tomography detected a purulent mass in the left subphrenic space, which extended with a massive pyothorax and resulted in the collapse of the left lung. Immediate surgery was elected, followed by the patient suffering a second cardiac arrest at the admission in the operating theater. External cardiac massage was performed, simultaneously with exploratory laparotomy, the drainage of a left subphrenic abcess and packing the subphrenic space due to a diaphragmatic bleeding; moreover, a left thoracotomy facilitated the evacuation of approximately two liters of fetid discharge. Two pleural drainage tubes were installed, as well as a nutrition jejunostomy catheter, because of an intraoperative suspicion of gastric fistula on the initial resection line. Postoperatively, the patient was admitted in the Intensive Care unit in severe septic shock and resuscitated cardiopulmonary arrest. After 48 hours, during a second surgery for depacking the subphrenic place, a fistula was discovered on the gastric resection line, right beneath the esogastric junction; a double-lumen suction tube was placed, with active intraluminal and passive subphrenic drainage. The patient had a slow and favourable evolution, undergoing extended antibiotherapy, volemic reequilibration, maximum supportive treatment, kinesiotherapy, nutrition via the jejunostomy catheter and chest drainage by active suction. 21 days after the surgery, a gastrografin examination was conducted, which evidentiated a superior gastric fistula with a diameter of approximately $1.5 \mathrm{~cm}$. A stent placement was performed by superior digestive endoscopy; the device remained in use for five weeks. Conclusion: Gastric fistula post-sleeve-gastrectomy is a severe complication correlated with this surgical procedure, due to its important implications on the patient's evolution, especially because of morbid obesity and associated comorbidities.

Keywords: sleeve-gastrectomy, gastric fistula, morbid obesity

115. Challenging Diagnosis and Treatment in a Rare Pelvic
Tumour
Turturea I Maria, Assistant Lecturer Claudia Stoica
"Carol Davila" University of Medicine and Pharmacy, Bucharest,
Romania

Background: Diseases within the pelvian space present multiple origins and clinical manifestations due to the great variety of anatomical structures. The ethiopathogeny involved could be both tumoral and inflammatory. As far as differential diagnosis is concerned, the clinician has to require the appropriate investigations and practice the most adequate procedures so that the surgical approach would be the correct one. The curative decision has to be taken intraoperatively. Case presentation: We present the case of a 36-aged patient who had multiple pelvian inflammatory processes after the removal of a sterilet. She was admitted to hospital with an abdominal CT which raised suspicion of pelvic tumour of uncertain origin. The symptomatology occurred 2 months ago with lumbar and left iliac fossa pain. The abdominal echography and the urologic consult indicated right ureterhydronephrosis of fourth grade, requiring cystoscopy and setting of a cook sonde. A urinary bladder mucosa biopsy was obtained. The imagistic aspect of the pelvic tumour revealed it was amprenting the urinary bladder and the sigmoid. The surgical intervention was decided and there was practiced an exploratory celiotomy discovering a pelvic inflammatory pseudotumoral formation of significant spread with adherence of the sigmoid to the urinary bladder. Evacuation of the pelvian collection was realized for the bacteriologic and cytologic exam purpose, also multiple peritoneal drainage and the biopsy of the pseudoinflammatory tumour from its junction side with the urinary bladder posterior wall. The postoperative evolution was favourable. Conclusion: The histopathological exam after cystoscopy revealed only inflammatory and granulation tissue as well as for the histopathological exam from the pelvian pseudotumoral tissue formation. Moreover, the last one indicated the characteristic aspect for Actinomyces Israeli bacteria colonies without neoplastic infiltration. So, the intraoperative decision to practice only biopsy and not to perform resection was the correct one. The intraoperative aspect could have led to total pelvectomy but the operator chose to obtain biopsy and ask for more investigations before practicing this radical cure. This case impresses through its unexpected particularity- Actinomyces inflammation and the necessity of the surgical differential diagnosis.

Keywords: pelvian pseudotumoral inflammmatory process, differential diagnosis, Actinomyces colonies

\section{Combination of Cells Technologies in the Treatment of} Chronic Gastric Ulcers

Hutsuliak V. Yurii, Professor Petrushenko V.V., Assistant Lecturer Radoga I.V., Assistant Lecturer Grebeniuk D.I. Vinnytsia National Medical University n.a. M.I. Pirogov, Vinnitsa, Ukraine

Background: Chronic stomach ulcer for many decades remains an issue of gastroenterology and surgery. The solution of this problem needs to go beyond the traditional methods of treatment. The possibility of local stimulation of reparation with the use of cell technologies is actively discussed in modern literature and is confirmed by the large number of randomized studies. Materials and methods: The study included 48 Wistar rats, both sexes under the age of 1 year and weighing 120 to $220 \mathrm{~g}(186 \pm 17 \mathrm{~g})$. All rats were divided into 3 groups. The first group was not treated acetate ulcer, the second group of acetate ulcer + local injection of lipoaspirate, third group of acetate ulcer + local injection of platelet-rich plasma. All rats on the first, seventh, and fourteenth day conducted measurements of ulcer area and morphological study. Results: The results of the experiments were evaluated at 7 and 14 days from the moment of stimulation of the reparationmeasurements of the area of the ulcers, and also took the material for morphological examination. The results indicate that stimulation of regeneration processes in ulcerative defects of the anterior wall of the stomach by any of the performed methods allows to significantly $(p<0,01)$ to accelerate the process of healing of ulcers, namely, to reduce their area. The results of the performed macro- and microscopic studies indicate that the plasma enriched with platelets stimulates the activity of fibroblasts and vasoconstriction in the young connective tissue, which improves the oxygenation of the ulcer and promotes cellular proliferation, differentiation and accelerating the maturation of the connective tissue and healing of the ulcer. Conclusion: The 
reparative effect of the local application of platelet-rich plasma, which is manifested by faster epithelization and restoration of the muscular layer, is significantly better $(p<0.01)$ than with the use of autologous purified lipoaspirate.

Keywords: chronic gastric ulcer, healing, lipoaspirate, platelet- rich plasma

117. Evaluation of Capsular Contracture: Software-Based Objective Analysis

Mangiabene - Alessandro, Professor Carmine Alfano

University of Perugia, Perugia, Italy

Background: Adverse capsular contracture (ACC) is the most frequent and problematical complication after breast implanting surgery. Its etiopathogenesis remains unclear, as well as its unpredictable evolution and difficult treatment. The evaluation of this condition has been performed for a long time only via clinical palpation, whose results sum up by Baker's well-known four classes. Nonetheless, newer objective methods are nowadays available to assess its grade, albeit not routinely used yet. This work essentially aims to submit and support one of these breaking techniques, irrespective of palpation's intrinsic subjective limit, therefore more suitable for scientific purpose: mammary compliance measurement and its further software-based analysis. Materials and methods: A prospective study was conducted in 48 patients who underwent mammary reconstruction by breast implanting over the last 4 years in our Department of Plastic and Reconstructive Surgery at the University of Perugia. Follow-up visits were scheduled at 1, 4, and 12 months. Capsular contracture was evaluated using concurrently the traditional palpatory method, applanation tonometry (another objective technique), and measurement of mammary compliance with the innovative software-backed device Tonometer Compliance System 1.1. Results: Data from our series reveal a good match between mammary compliance values, and those for applanation tonometry and Baker's clinical grading. Conclusion: Mammary compliance measurement represents an absolutely effective objective method of ACC rate evaluation. Due to its high sensitivity it is well-suited to both long-term follow-up studies and close monitoring of therapeutic interventions. Tonometer Compliance System 1.1 revealed to be an excellent computerized system for calculating and working through compliance itself: it allows elimination of the traditional clinical and subjective evaluation of capsular contracture; it is easy to use, with readily reproducible results and graphs simple to interpret; all the work is done by a software, therefore results may be digitally recorded and filed for future comparisons.

Keywords: breast implanting, capsular contracture, objective assessment, mammary compliance, software-based analysis

118. Fundal Scar Pregnancy Following Surgical Termination of Pregnancy in Patient's History

Udre A Santa ${ }^{1,2}$, Salajeva Vladimirovna Viktorija', Lecturer Zane Krastina ${ }^{1,2}$

University of Latvia Faculty of Medicine, Riga, Latvia, 2pauls Stradins Clinical University Hospital, Riga, Latvia

Background: Uterine perforation is an uncommon complication of first-trimester suction curettage. Ectopic pregnancy at the perforation site is a hypothetically probable but very rare complication. This clinical case presents fundal scar pregnancy likely due to myometrial injury following uterine curettage in patient's history. The routine use of early ultrasonography is very important, before serious complications occur. Use of early ultrasonography and $\beta$-human chorionic gonadotropin levels measurements are significant to detect and follow up these cases. Case presentation: A healthy 39-year-old Asian woman, gravida 3 para 1 was presented to the hospital at 8 weeks pregnancy. Vaginal ultrasonography showed an empty uterine cavity with a thickened endometrium and well-circumscribed mass was seen fundally which was a gestational sac (130 $\mathrm{mm}$ in diameter) with fetal pole inside and a positive fetal cardiac activity - fundal scar pregnancy with bulging of the uterus. Patient's first pregnancy was a normal vaginal delivery with no complications, the second pregnancy was interrupted with a legal surgical abortion five years ago. After oneweek ultrasound demonstrated the stop of fetal growing and bradycardia, $\beta$-hCG (beta human chorionic gonadotropin) measurements at 9 weeks 2 days- $8311 \mathrm{mU} / \mathrm{ml}$, after 3 days 8454 $\mathrm{mU} / \mathrm{ml}$. At 10 weeks and 2 days there were signs of potential missed spontaneous abortion. At 11 weeks and 5 days patient complained about severe bleeding, next day $\beta-\mathrm{hCG}-47 \mathrm{mU} / \mathrm{ml}$. Two days later it was complete missed abortion: no fetal pole, uterus size normal (LL $66,7 \mathrm{~mm}$, AP 40,8, length $56 \mathrm{~mm}$ ), endometrium $4,4 \mathrm{~mm}$ and in fundus area there was a small shadow in myometrium (in region there was fundal scar pregnancy). Conclusion: Embryo implantation in a previous iatrogenic uterine perforation is rare but potentially life-threatening condition as for all ectopic pregnancies. However, a delay in either diagnosis or treatment can lead to uterine rupture, hysterectomy, and significant maternal morbidity. In this lucky case treatment was not needed, because of missed abortion. Close monitoring with ultrasonography and $\beta$-hCG measurements can provide a safe possibility to follow up and avoid invasive treatments.

Keywords: ectopic pregnancy, scar pregnancy, abortion complication, uterine perforation

\section{Groin Flap Used in Sequelar Lesion After Third Degree Burn} of the Hand in a Young Female Pacient

Contiu N. Loredana - Maria', Dan MC Elena-Calina',2, Assistant Lecturer Dr. Dorobantu Dorin², Assistant Lecturer Dr. Trambitas Cristian², MD PhD Dr. Bumbu Anamaria Victoria ${ }^{2}$

'University of Medicine and Pharmacy, Tîrgu Mures, Romania, ${ }^{2}$ Clinical Emergency Hospital, Tîrgu Mures, Romania

Background: Burn injuries are very frequent, leading to longlasting physical, functional, aesthetic, psychological and social consequences; the longer it takes for the burn wound to heal, the more serious are the sequelae. Reconstruction of tissue defects in hand burns is a challenge for any plastic surgeon. To correct the deformities the most common choice is the Z-plasty technique, followed by Z- plasty + full thickness skin grafts, full thickness skin grafts, tissue expansion, Z- plasty + local flaps, flaps (local fasciocutaneous, radial forearm) and direct closure. Case presentation: We describe the case of a 24 -year-old woman patient, presented to the Plastic Surgery Department with sequelae lesions after suffering at the age of 2 bilateral third degree burns on hands. During her life, she suffered multiple surgical interventions, in which where performed full thickness skin grafts. A decision was made to cover the defect of the volar part of the left hand, using a groin flap because we considered that another full thickness skin graft is not a solution anymore in this case. After the surgery, the patient remained hospitalized to be monitored for the development of any postoperative complications such as infection, flap necrosis. Conclusion: The surgical treatment of burns sequelae is a difficult process and often requires the application of a combination of surgical techniques. Groin flap has long been accepted as a safe, easy and reliable option for the reconstruction of soft tissue defects. Despite of long hospital stay, uncomfortable position and number of operations, results obtained from groin flap are assured and satisfactory.

Keywords: groin flap, burns sequelae, hand defect

\section{Is a Middle Cerebral Artery (MCA) Bypass Efficient in a} Moyamoya Disease Case?

Elsayed R Abdallah', MD Dominik Seggewiß ${ }^{2}$

"Carol Davila" University of Medicine and Pharmacy, Bucharest, Romania, ${ }^{2}$ Charité Mitte, Berlin, Germany 
Background: Moyamoya ("Puff of smoke" in Japanese) is a rare, progressive cerebrovascular disorder caused by blocked arteries at the base of the brain with particular involvement of the circle of Willis and the arteries that feed it. It was firstly described in Japan in the 1960s hence its name and currently the highest incidence can be found there ( 0.35 case per 100,000 people). The peak of the disease is in the first decade of life affecting females nearly as twice as males (female-to-male ratio is 1.8:1). Moyamoya disease can be congenital or acquired, but only about $10 \%$ of the cases are familial. Case presentation: A 34-year old female patient presented to the neurosurgery department of the Charité Mitte, Berlin Cermany in September 2017, with an intermittent tingling and hypoesthesia in the left side of the body. The patient was diagnosed in 2011 with Moyamoya disease, located in the right hemisphere. After 5 years, she had a right STA-MCA (Superior Temporal Artery - Middle Cerebral Artery) bypass in 2016 as a revascularization attempt. Postoperatively she suffered from a transitory ischemic attack in the anterolateral part of the right frontal lobe supplied by the right MCA, causing a noticeable decrease of blood supply to the right hemisphere. Based on the history and anamneses, she received an angiography that showed a bilateral Moyamoya disease with a bilateral high-grade stenosis in the ICAs (Internal Carotid Arteries) and proximal MCAs. Based on those finding, a right ECA (External Carotid Artery) - MCA bypass was performed using a graft of the right radial artery of the patient. The operation was done successfully and the patient was monitored for 10 days in the department, as she was receiving physiotherapy for the Post-OP rehabilitation. It's recommended for her to present herself for further check-ups as there is a possibility of further operations due to the current presence of the disease in the left hemisphere as well. Conclusion: MCA bypasses serve an efficient approach to relieve the symptoms of Moyamoya syndrome, but due to the constant evolutionary course of the disease, the patients tend to be operated more than once.

keywords: MCA, STA, ECA, ICA, moyamoya, neurosurgery, brain

121. Management of Complex Shoulder Fractures in Polytrauma Patient.

Porcisteanu G Ceorgiana-Nicoleta', Lecturer Ioan Cristescu2, MD Daniel Vilcioiu ${ }^{2}$

'"Carol Davila" University of Medicine and Pharmacy, Bucharest, Romania, ${ }^{2}$ Clinical Emergency Hospital, Bucharest, Romania

Background: Complex shoulder fractures can result from high energy trauma, such as motor vehicle accidents, contact sports, falls, etc. and may concern all three component bones of the articulation. A constantly expanding range of reconstructive options and the possibly better outcome of the treatment have been extending the indications for surgical therapy of complex shoulder fractures. As a result, management decisions are becoming increasingly complicated, in an attempt to provide the best possible treatment for each individual patient. Case presentation: A 33-year-old female patient is transferred to the Emergency Hospital Floreasca following a motor vehicle accident to undergo specialty treatment. Upon arrival at the Emergency Department, she presents numerous signs of injuries: frontotemporal concussion with significant ecchymoses, left hemithorax trauma with minimum subcutaneous emphysema, osseous crepitus and multiple excoriations, multiple rib fractures on the right side and minimum bilateral hemothorax. She accuses pain and functional impotence in the left shoulder. Highly displaced transverse midshaft fracture of the left clavicle, comminuted surgical neck fracture of the left humerus and glenoid fracture-avulsion are diagnosed following radiological examination. The patient is admitted to Orthopaedics and Traumatology Department and is treated surgically by early total care (ETC), with minimally open reduction and intramedullary fixation for the clavicle fracture and with open reduction and internal fixation with Philos plate for the humeral fracture. The scapular fracture did not require fixation. No intraoperative complications occurred. The surgical treatment allowed early and safe postoperative rehabilitation. Conclusion: The management of the polytraumatized orthopaedic patient may represent a challenging issue, the crucial step being the allocation of the right surgical principle-early total care (ETC) vs Damage control orthopaedics (DCO). The particularity of this case lies in the simultaneously displaced fractures of the clavicle and ipsilateral humeral neck caused by high energy trauma. The greater instability of the shoulder joint directed the medical decision towards an immediate operative treatment of both fractures, which resulted in better union time and faster recovery of the shoulder functionality.

Keywords: shoulder fractures, clavicle fracture, humeral fracture, polytrauma, intramedullary fixation, internal fixation, early total care

122. Nanofabricated Poly(vinyl alcohol) Meshes in the Future of Abdominal Hernia Treatment

Voniatis G Constantinos, Academician Daniella Fehér, Academician Kristof Molnar, Academician Angela Jedlovszky-Hajdu, Professor Miklos Zrinyi, Professor György Wéber

'Semmelweis University, Budapest, Hungary

Background: Abdominal hernia is an intestinal loop protrusion through a defect in the abdominal muscular wall. As one of the most frequently performed operation in general surgery, management of abdominal hernias has undoubtedly evolved throughout the years, but has it reached its full potential? Up to date protocols suggest as first choice treatment, a laparoscopic hernioplasty involving implantation of a surgical mesh to close the defect and treat the hernia, but surprisingly currently applied surgical meshes are not as perfect as they seem. Intraperitoneal fixation of dry, woven, non-biodegradable meshes induce complications like tissue adhesion, mesh deformation or even foreign body reactions far more often than expected. Electrospinning is a versatile technique, popular in nanotechnology used for tissue engineering and scaffold production. Our objective was utilizing electrospinning to produce a bio-compatible, bio-degradable surgical mesh that could one day serve as a potential replacement for the currently applied surgical meshes. Materials and methods: Non-woven, PVA scaffolds were produced via electrospinning of 9 W\% PVA and IM GDA solutions. Post electrospinning processing included folding, compression and formation of cross links via scaffold immersion in $2 \mathrm{M} \mathrm{HCl}$. Scaffolds were sterilized with $\mathrm{ClO}_{2}$ stored in PBS while incubated at 37 OC. In Vivo application was examined on wistar rats $(n=60)$. A 1st group, served as a preliminary bio-compatibility study with PVA scaffolds implanted and fixed intraperitoneally. In a 2nd and 3rd group, abdominal defects $(2 \times 2 \mathrm{~cm})$ were created and PVA scaffolds (D: $2.5 \mathrm{~cm})$ were used to repair them, while a th group served as control with the defects repaired conventionally. Animals were dissected on the 7th, 14th, 28th, 90th and 180th postoperative day. Scaffolds were evaluated macroscopically and microscopically as well. Results: None of the animals behaved differently or showed signs of complications following the operation. All animals survived until termination and dissection revealed no aberrations on the scaffolds or the surrounding tissue. Adhesion formation was surprisingly found along the suture material rather than the scaffolds themselves. Histological examinations revealed scaffold integration into the host tissue. Conclusion: Considering our positive results, nanofabricated PVA scaffolds could in the future serve as viable candidates for abdominal hernia treatment.

Keywords: abdominal hernia, surgical meshes, electrospinning, poly (vinyl alcohol)

123. Orthotopic Kidney Transplantation

Costache R. C. Alexandru-Dan', Covic A Alexandra', Tarcau $S$ Bogdan- Marian', Dobreanu M.A. Stefan Dorin', Lecturer 
Corneliu Moroșanu',2, Lecturer Simona Hogaș ${ }^{1,2}$, MD Angelica Gramaticu ${ }^{2}$

"Gr. T. Popa" University of Medicine and Pharmacy, Iași, Romania,

2"Dr. C. I. Parhon" Clinical Hospital, Iași, Romania

Background: One of the most significant health issues with a rising prevalence in recent years is the end stage chronic kidney disease (ESRD). The most effective renal replacement therapy remains the kidney transplantation. Case presentation: We are reporting the case of a 22-year-old female patient with ESRD on hemodialysis for 12 years, who underwent a kidney transplant procedure in January 2016, from a deceased donor. The patient was suffering from dwarfism and also required a nephrectomy on a sole kidney due to a vesicoureteral reflux which has caused a megaureter and frequent urinary tract infections. The other kidney had also been removed at a younger age for the same reasons. Therefore, the choice was for the orthotopic approach, despite the procedure being normally performed in a heterotopic manner. The renal transplantation was successful with no surgical complications, followed by a rapid decrease in serum creatinine levels from 9,2 to $0,5 \mathrm{mg} / \mathrm{dl}$. Afterwards, the patient developed two episodes of acute cellular rejection that required Methylprednisolone bolus therapy. Eleven months after the transplant procedure, the patient suffered from a new acute graft dysfunction episode with a rise in serum creatinine level up to $3.1 \mathrm{mg} / \mathrm{dl}$ (estimated glomerular filtration rate of $26.9 \mathrm{ml} / \mathrm{min} / 1.73 \mathrm{m2}$ ). After excluding other specific causes, a renal biopsy was performed, which showed signs of humoral rejection that was treated with plasmapheresis and iv immunoglobulin, followed by an increase of $13.8 \mathrm{ml} / \mathrm{min} / 1.73 \mathrm{~m} 2$ in eGFR. Conclusion: The particularities of this case are the orthotopic approach of renal transplantation, the rapid onset of humoral graft rejection, despite the high immunological compatibility and the increase in eGFR after treatment.

Keywords: orthotopic kidney transplantation, graft rejection, chronic kidney disease

124. Surgical Treatement of Renal Cyst Discovered Accidentally During Pregnancy

Tanase M.A Adina', Valentina Paraschiva M Huțanu², Otilia Andreea L Petcuța ${ }^{2}$, Gabriel - Valentin MA Tanase ${ }^{2}$, MD Phd.Dr Tanase Adina'

"Cuza Voda" Clinic Hospital, Iași, Romania, z"Gr. T. Popa" University of Medicine and Pharmacy, lasi, Romania

Background: In case of kidney cysts associated with pregnancy, it is not clear which treatment is the best one. It is important to pay more attention to the possibility of infection during the pregnancy. It is well known that the urinary tract infection develops during 24-28 weeks of pregnancy, due to the fact that the uterus is growing and will obstruct the urinary tract. This process will modify the size of the cyst and it will help in developing a new infection. Case presentation: We report the case of a pregnant woman, checked up by an OB specialist, with no clinical evidence of diseases during her lifetime, until 27 years old, that was diagnosed at 22 weeks of pregnancy, after a routine check-up, for hypertension with a $17 / 17 / 22 \mathrm{~cm}$ retroperitoneal maternal mass, which led to a urological consult. The diagnosis was gigantic superior polar renal cyst. A percutaneous drainage technique was performed and $2000 \mathrm{ml}$ of clear fluid was extracted; the ultrasound evaluation revealed a transonic mass with internal septa $(14 / 17 \mathrm{~cm})$, that changed the anatomy of the left kidney that was deviated closer to the aorta. Shortly after birth, she presented signs of malfunctioning nephrostomy and impossibility of creating a communication between the drained cavity and the pyelocaliceal system, with 2 recurrent mass cystic lesions forming at 2 months after delivery. In the end, a total laparoscopic nephrectomy was performed. Conclusion: Most cysts on the kidneys are harmless and usually if the cyst on the kidney is smaller than $3 \mathrm{~cm}$, no treatment is needed. However, for a pregnant, the condition may be different. Actually, more considerations are needed to be known by the pregnant woman. The therapeutic approach of kidney cysts discovered during pregnancy is controversial. Particular attention should be paid to the risk of infection and sepsis.

Keywords: renal polar cyst, secondary hypertension, pregnancy, nephrostomy

125. The Management of Cesarean Scar Ectopic Pregnancy Following Treatment with Methotrexate

Salajeva Vladimirovna Viktorija',2, Udre A Santa', Lecturer Zane Krastina ${ }^{2}$, MD Baiba Lesina-Korne ${ }^{2}$

'University of Latvia, Riga, Latvia, 2Pauls Stradins University Clinical Hospital, Riga, Latvia

Background: Methotrexate injection is an effective, but also a new method to resolve the Caesarean scar pregnancy. There is no relevant guidelines how to rule clinical incidents and what is the right level of serum $\beta$-hCG (beta human chorionic gonadotropin), when patient is safe from bleeding and hysterectomy risk. For the last few years the number of Cesarean sections has increased therefore Cesarean scar pregnancies are becoming more common in clinical practice. Case presentation: A 30-year-old woman was referred to a hospital gynecological department from a local medical clinic with diagnosed Cesarean scar ectopic pregnancy graviditas III in septima 4+6. The serum $\beta$-hCG level was 64268,0 $\mathrm{mIU} / \mathrm{mL}$ (milli-international units/milliliter). As the patient was hemodynamically stable and asymptomatic, a single dose of $85 \mathrm{mg}$ methotrexate was given to the patient perorally. Gynecological ultrasound had shown that pregnancy was still progressing. Under local anesthesia during transabdominal ultrasound a needle was inserted into the ectopic gestational sac, and a $60 \mathrm{mg}$ methotrexate solution was injected into the fetus. The patient's serum $\beta$-hCG level decreased to $49589,0 \mathrm{mIU} / \mathrm{mL}$ on the ninth day after management. Within 32 days of the administration of methotrexate the patient developed vaginal bleeding, requiring emergency admission - the procedure of arteria uterina dextra and sinistra embolization was performed. Later $800 \mu \mathrm{g}$ (microgram) Misoprostol was given vaginally. As the gestational sac was observed through gynecological ultrasound, clinicists decided to have a dilation and curettage. The patient's serum $\beta$-hCG level declined to $95,0 \mathrm{mIU} / \mathrm{mL}$ on the fourth day after the uterine artery embolization. Conclusion: The main disadvantage of MTX therapy is that trophoblastic tissue is not detaching from the Caesarean scar, therefore the risk of bleeding becomes higher, especially when placenta's good vascularized tissue starts to degenerate. Usually, to have safe consequences, clinicists use Misoprostol to induce uterine contraction. In this case, the patient was discharged from the hospital with a high serum $\beta$-hCG level and without Misoprostol treatment.

Keywords: $\beta$-human chorionic gonadotropin, Cesarean section, ectopic pregnancy, methotrexate, misoprostol

126. The Management of Obturator Hernia: A Challenging Task. Moldovanu R Stefana Eugenia', Stefanuca M Iuliana', Doca L Ana-Maria', Ciobotariu M.C. Iulia Mihaela', Assistant Lecturer Lili Lozneanu², Assistant Lecturer Lacramioara Perianu ${ }^{3}$, Lecturer Nutu Vlad², Lecturer Dan Andronic ${ }^{2}$ "Gr. T. Popa" University of Medicine and Pharmacy, Iași, Romania, "Department of Surgery, "Gr. T. Popa" University of Medicine and Pharmacy, Iași, Romania, 3Department of Anatomy, "Gr. T. Popa" University of Medicine and Pharmacy, Iași, Romania

Background: Obturator hernia $(\mathrm{OH})$ is a rare disease with an incidence of 0.05 to $1.5 \%$ from all the hernias. It is a type of pelvic hernia in which a bowel segment protrudes through the obturator foramen, adjacent to the obturator vessels and nerve, that fails to reduce spontaneously. The $\mathrm{OH}$ diagnosis and management is always challenging because the signs and symptoms are non- 
specific. Thus, the diagnosis delay is common and leads to high mortality and morbidity. In most cases the diagnosis is made preoperatively, during abdominal exploration for an intestinal obstruction. Thereby, they have the highest mortality rate of all abdominal wall hernias at between $13 \%$ and $40 \%$. Case presentation: We present the case of an 88-year-old woman who was admitted in emergency with signs of intestinal obstruction: intense abdominal pain irradiating to right inguinofemoral region, palpable painful mass in groin area and Howship-Romberg sign positive. The biological exam revealed leukocytosis (11000/mm3), elevated CRP ( $45 \mathrm{mg} / \mathrm{dL})$. A CT-scan was performed in emergency and revealed a strangulated right obturator hernia associated with right femoral hernia. An exploratory laparoscopy was performed. The diagnosis of strangulated obturator hernia was confirmed. A concomitant femoral hernia containing epiploon was also diagnosed. The small bowel was reduced and then, the hernia repair was performed by transabdominal pre-peritoneal (TAPP) procedure. To note that the small bowel was not ischemic at the end of procedure. The postoperative course was uneventful and the patient was discharged at day 7. Conclusion: $\mathrm{OH}$ is a rare disease but has to be considered as possible etiology for intestinal obstruction for old women. The laparoscopic approach allows a good inspection of inguinal and pelvic area and allows the diagnosis and treatment of $\mathrm{OH}$.

Keywords: obturator hernia, intestinal obstruction, endoscopic hernia repair, TAPP

127. The Role of Histopathology and Immunohistochemistry in the Diagnosis of Neuroendocrine Biliary Duct Tumor Baicu C Andrei Marius', MD PhD Mirela Patricia Boeti Sirbu², MD Tintarescu Gabriel3, MD Mirela Boros², MD PhD Vlad Herlea², MD Ioana Niculina Luca², MD Vladislav Brasoveanu ${ }^{2}$

"Carol Davila" University of Medicine and Pharmacy, Bucharest, Romania, ${ }^{2}$ Fundeni Clinical Institute, Bucharest, Romania, 3"Sanconfind" Medical Centre, Câmpina, Romania

Background: Neuroendocrine tumors (NETS) are benign or malignant histopathological entities, secretory or non-secretory, developed in an organic structure (pancreas, ileum, liver, lung etc.) or more (carcinoids). These are slow growing tumors, difficult to diagnose in early stages. NETs represent a small fraction (approximately $3 \%$ ) of all neoplasms and their occurrence is influenced by genetic mutations. These tumors are found mostly in the digestive system (54.5\%), but their incidence is low at the biliary tree level $(0.5-2 \%)$ and the differential diagnosis must be done with hilar cholangiocarcinoma (Klatskin tumors). In the following clinical case, there are highlighted the difficulty of preoperative and intraoperative diagnosis of NETs at the biliary tract level, the need of complex surgical resection for such a tumor and the importance of histopathology and immunohistochemistry examinations in their diagnosis. Case presentation: A 60-year-old woman, cholecystectomised in 2004, is hospitalized in 27.05.2013 with jaundice and abdominal pain. MRI examination shows a mass $(25 \mathrm{~mm})$ at the hepatic duct confluence with compression on the right hepatic duct that is diagnosed as a Klatskin tumor grade IIla. During surgical tumor dissection, the physicians encounter tumoral invasion of the portal vein and they decide to perform common biliary tract resection en-bloc with extended right hepatectomy (including segment IV), segmentary portal vein resection with porto-portal anastomosis and cholangiojejunostomy (Roux-en-Y technique). The histopathological examination of the surgical specimen shows small cells with a $1 / 10$ high-power field mitotic index, resembling a neuroendocrine tumor grade I (NET-G1). Immunohistochemistry examination confirms the NET-G1 diagnosis, the tumor being positive for Chromogranin A, NSE and Ki67, specific for this type of neoplasm. Postoperatively, the patient's evolution is favorable and she is discharged after 5 days. Oncological treatment is not recommended. The patient was dropped out from oncological follow-up in 2015, being disease-free and alive until present.
Conclusion: Neuroendocrine tumors of the biliary tree are uncommon comparative with Klatskin tumors. An aggressive approach is justified for the neuroendocrine tumors even when a local extension is discovered intraoperatively because they pose a favorable prognosis after complete resection with no need of adjuvant chemotherapy. In order to properly diagnose them, an immunohistochemistry examination must complete the histopathological result.

Keywords: neuroendocrine tumors, Klatskin tumors, hepatic duct confluence, surgical resection, immunohistochemistry, histopathology

128. The Surgical Approach of Epilepsy and the Neuro-Anatomic Consequences of Corpus Callosotomy

Buzatu S Valentina Alina, Giuroiu C Florin, Iordache A Andrei, Vasile I Mirela-Elena, Assistant Lecturer Ioan Bulescu

"Carol Davila" University of Medicine and Pharmacy, Bucharest, Romania

Background: Epilepsy comprises a spectre of long term neurologic disorders, that vary from severe, debilitating, life-threatening, to benign forms. The epileptic fits are caused by inherent neuronal disturbance and they classify as either convulsive (60\% of cases) and (or) non-convulsive ( $40 \%$ of cases). A particular form, that is difficult to treat and debuts in childhood is the Lennox-Gastaut syndrome (LCS). LCS features a triad of frequent seizures of multiple natures (tonic-clonic, atonic and atypical epileptic absence). The electroencephalogram (EEG) pattern reveals slow, less than $3 \mathrm{~Hz}$ spike-wave complexes and the affliction of cognitive functions, from moderate to severe. Case presentation: Male patient aged 6 presents to the emergency room for drop-attack, tonic-clonic and atonic seizures. The electroencephalographic examination signals the presence of frequent slow spike-wave complexes and multifocal spike waves, thus the patient is diagnosed with LCS. The magnetic resonance imaging (MRI) examination shows no abnormalities. Despite following an antiepileptic pharmaceutical treatment (valproate, topiramate, zonisamide, clobazam) and a ketogenic diet, the seizures have remained uncontrollable. The patient undergoes the surgical corpus callosotomy in the anterior $2 / 3$ rds (the sectioning of the anterior $2 / 3$ rds of the corpus callosum fibers). Post-operative the evolution is favorable, the patient becomes seizure-free, however after 3 months the fits relapse. EEG examination 10 months postoperatory shows frequent generalized spike waves and breakthrough generalized rapid activity. The seizures respond neither to the additional stimulation of the vagus nerve, nor to the pharmaceutical treatment. The patient is operated on for the second time. The surgical intervention consists of the sectioning of the splenius fibers, therefore achieving a total corpus callosotomy. Although total corpus callosotomy associates risks and more severe complications, such as the disconnection syndrome, the epileptic fits no longer occur. Subsequent investigation highlights the normalization of the EEG. At the moment, the patient follows a therapeutic program that is designed to improve on the sensorimotor incoherence. Conclusion: The presented case endorses the surgical approach of medicinally nonresponsive epileptic forms, in spite of the associated risks.

Keywords: epilepsy, Lennox-Gastaut syndrome, corpus callosotomy

\section{Using the Nonmicrosurgical Techniques for Reconstruction} of the Amputated Thumb

Tofan C Remus-Andrei, ${ }^{1,2,3}$, Para A Adina Ceorgiana ${ }^{1,3}$, Daniel-George C Boicu', Lecturer Mihaela Pertea ${ }^{1,2,3}$

"Gr. T. Popa" University of Medicine and Pharmacy, Iassi, Romania, ${ }^{2}$ Clinic of Plastic Surgery and Reconstructive Microsurgery, lași, Romania, 3"St. Spiridon" Emergency Clinical Hospital, Iași, Romania 
Background: The aim of this study is to evaluate the outcomes of the reconstruction in the emergency of the amputated thumb using isolated or associated homodigital and heterodigital flap techniques in those situations when microsurgical replantation is not possible due to local or general conditions. The study is based on the evaluation of the functional (thumb length, sensitivity, motility active joint movement and cortical reintegration) and esthetic outcomes. Materials and methods: 32 patients aged between 18 and 65-year-old, with complete posttraumatic amputation of the thumb at the level of interphalangeal joint or proximal phalanx were evaluated. The aim of reconstruction was to achieve a functional thumb of proper length, good sensitivity, motility and cortical reintegration of the new pulp. In 8 cases, the reconstruction was done using the Mantero-Bertolotti technique with an 0'Brien flap, and in 6 cases the use of a single heterodigital neurovascular Littler flap was sufficient. The association of the two techniques with a Littler's flap instead of the O'Brien flap in the Mantero-Bertolotti reconstruction was the choice in 14 patients. In other 4 cases, the reconstruction algorithm included the use of a Foucher (cerf-volant), Simonetta or Hueston flap. Results: The results have been evaluated based on age, injury complexity, size of the flap, mobility, sensitivity, cortical reintegration of the new pulp. The best average range of motion of the new thumb in those cases in which we applied the associated technique (Littler's flap instead of the 0'Brien flap in the Mantero-Bertolotti reconstruction) using Kapandji score (8 score). Regarding the sensibility, we achieved a protective sensibility of the new thumb. At the two-point discrimination test (2PD test), the results were between 7-11 mm. At the light touch deep pressure test ( $S W$ test), all the patients felt the blue monofilament and 24 out of 32 felt the violet monofilament. Conclusion: The use of isolated or combined homodigital and heterodigital, flap techniques is a proper choice for reconstructing the amputated thumb in those cases when the microsurgical replantation is not possible. The microsurgical replantation remains the gold standard in thumb amputation.

Keywords: thumb, amputation, flap

130. Year Results Demonstration of Subbasal Nerve Fiber Changes after the Flapless Femtosecond Refractive Procedure SMILE Versus Femtosecond LASIK
Veinberga Valereevna Dineja', Mescerjakova Julija', MD Andrei Solomatin ${ }^{2}$, Associate Professor Igor Solomatin², MD Jana Gertnere ${ }^{2}$, MD Maksim Solomatin ${ }^{2}$

'University of Latvia, Riga, Lativa, ${ }^{2}$ Dr. Solomatin Eye Rehabilitation Clinic, Riga, Latvia

Background: The absence of corneal subbasal nerve fibers can cause multiple disorders for example dry eye, hypaesthesia and neurotrophic epitheliopathy. These disorders can affect the patient anywhere from months to years post-surgery. The regeneration of the nerve fibers depending on the pre-op condition of the eye and the type of surgery employed. In our recent study, we sought to quality and quantitatively compare corneal subbasal nerve fibe regeneration variances after SMILE or Femto-LASIK. In this report we present the details, findings and results of our study. Materials and methods: Two groups of myopic patients, range 19-40, were selected for this investigation: 20 patients (40 eyes) for SMILE procedure and 20 patients (40 eyes) for Femto-LASIK procedure. It was using the same $500 \mathrm{kHz}$ femtosecond laser for SMILE procedure and bladeless flap creation Femto-LASIK procedure. Corneal subbasal nerve regeneration data was measured using confocal microscopy (Confoscan 4, Nidek) preoperatively, 1, 3, 6 and 12 months post operatively. Results: The study enrolled 80 eyes. The quantitatively compare nerve fiber regeneration differences, the nerve fiber lenght to Femto-Lasik pre-op (1130.85 $422.42 \mathrm{SD})$ and

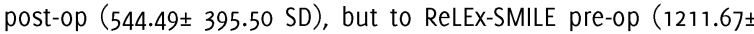
467.34 SD) and post-op (601.16 494.76 SD). Lenght density to Femto-Lasik pre-op (13881.20 5311.39 SD) and post-op (5756.84 4294.20 SD), but to ReLEx-SMILE pre-op (14728.61 \pm 5680.85 SD) and post-op (6001.16 $\pm 4094.76 \mathrm{SD})$. Number of beadings to Femto-Lasik pre-op (87.89 \pm 39.66 SD) and post-op (37.68 29.76 SD), but to ReLEX-SMILE pre-op ( $89.54 \pm 44.01$ SD) and post-op ( $42.80 \pm 29.50$ SD). The highest statistically significant difference $(p, 0.001)$ was found in following parameters; nerve fiber length, nerve fiber length density, number of beadings. No statistically significant difference was found in the NIDEK tortuosity parameter. Conclusion: Both surgical techniques cause regression in all nerve fiber parameters, SMILE procedure preserves trigeminal nerve density better than Femto-Lasik. Less time is needed for nerve fibers to regenerate after SMILE procedure comparing to FemtoLASIK.

Keywords: subbasal nerve fiber, SMILE, Femto-LASIK, NIDEK parameter 